\title{
Genetic testing and common disorders in a public health framework: how to assess relevance and possibilities
}

\section{Background Document to the ESHG recommendations on genetic testing and common disorders}

\author{
Frauke Becker ${ }^{1,26}$, Carla G van $\mathrm{El}^{2,26}$, Dolores Ibarreta ${ }^{3}$, Eleni Zika ${ }^{3}$, Stuart Hogarth ${ }^{4}$, Pascal Borry ${ }^{2,5,6}$, \\ Anne Cambon-Thomsen ${ }^{7}$, Jean Jacques Cassiman ${ }^{8}$, Gerry Evers-Kiebooms ${ }^{9}$, Shirley Hodgson ${ }^{10}$, \\ A Cécile JW Janssens ${ }^{11}$, Helena Kaariainen ${ }^{12}$, Michael Krawczak ${ }^{13}$, Ulf Kristoffersson ${ }^{14}$, Jan Lubinski ${ }^{15}$, \\ Christine Patch ${ }^{16}$, Victor B Penchaszadeh ${ }^{17}$, Andrew Read ${ }^{18}$, Wolf Rogowski ${ }^{19,20}$, Jorge Sequeiros ${ }^{21}$, \\ Lisbeth Tranebjaerg $^{22}$, Irene M van Langen ${ }^{23}$, Helen Wallace ${ }^{24}$, Ron Zimmern ${ }^{25}$, Jörg Schmidtke ${ }^{1}$ and \\ Martina C Cornel ${ }^{*, 2}$
}

European Journal of Human Genetics (2011) 19, S6-S44; doi:10.1038/ejhg.2010.249

Keywords: common disorders; genetic testing; predictive value; monogenic subtypes; public health

\section{INTRODUCTION}

\section{Background and purpose}

During the years prior to the turn of the century, scientific and medical attention for genetic disorders was mainly focused on understanding rare single-gene disorders, such as Huntington's disease, Duchenne muscular dystrophy, and cystic fibrosis (CF), as well as chromosomal abnormalities. The medical specialty of clinical genetics was established in the 1980s and 1990s in many European countries to diagnose these kinds of rare disorders and to counsel patients and families.

In recent years, the attention of the genomics and genetics research community has shifted toward understanding the basis of common complex disorders. Common diseases are diseases frequently encountered in health care. Some cases of common disorders are characterized by a strong influence of germline mutations in a single gene; these will be referred to as 'monogenic subtypes'. In many cases common disorders have a multifactorial etiology: they are caused by several genes and environmental factors, involving gene-gene and/or gene- environment interactions. We will use the term 'complex disease' to indicate diseases with variable etiology, including multifactorial etiology as well as monogenic subsets. When discussing 'susceptibility genes' in this document, we refer to genetic variants with low predictive value. We need to admit, however, that no generally accepted threshold for categorizing predictive value levels exists. Researchers nowadays study the myriad of genetic polymorphisms that have been identified during and since the Human Genome Project. The spectacular growth of genome-wide association studies ${ }^{1}$ has shed new light on which of these variants represent risk factors for common diseases. Understanding the pathogenesis and etiology, and finding new ways to prevent and treat those diseases are major challenges. The traits or diseases under study include coronary artery disease, myocardial infarction, stroke, peripheral artery disease, obesity, type 1 diabetes, type 2 diabetes, breast cancer, cervical cancer, colorectal cancer, prostate cancer, celiac disease, bipolar disorder, Crohn's disease, and many more. Should incorporation of these research results into current clinical and public health practice become

\footnotetext{
${ }^{1}$ Hannover Medical School, Department of Human Genetics, Hannover, Germany; ${ }^{2}$ Department of Clinical Genetics and EMGO Institute for Health and Care Research, VU University Medical Center, Amsterdam, The Netherlands; ${ }^{3}$ IPTS Institute for Prospective Technological Studies, Joint Research Centre, European Commission, Seville, Spain; ${ }^{4}$ Department of Social Sciences, Loughborough University, Loughborough, UK; ${ }^{5}$ Centre for Biomedical Ethics and Law, Katholieke Universiteit Leuven, Leuven, Belgium; ${ }^{6}$ Department of Medical Humanities and EMGO Institute for Health and Care Research, VU University Medical Center, Amsterdam, The Netherlands; ${ }^{7}$ Inserm, U 558, Department of Epidemiology, Health Economics and Public Health, University Paul Sabatier, Toulouse, France; ${ }^{8}$ Center for Human Genetics, University of Leuven, Leuven, Belgium; ${ }^{9}$ Psychosocial Genetics Unit University Hospitals, Katholieke Universiteit Leuven, Leuven, Belgium; ${ }^{10}$ Department of Clinical Genetics, St George's University of London, London, UK; ${ }^{11}$ Department of Epidemiology, Erasmus MC University Medical Center, Rotterdam, The Netherlands; ${ }^{12}$ National Institute for Health and Welfare, Helsinki, Finland; ${ }^{13}$ Institute of Medical Informatics and Statistics, Christian-Albrechts-Universität, Kiel, Germany; ${ }^{14}$ Department of Clinical Genetics, University Hospital, Lund, Sweden; ${ }^{15}$ Department of Genetics and Pathology, International Hereditary Cancer Center, Pomeranian Medical University, Szczecin, Poland; ${ }^{16}$ Guys and St Thomas' NHS Foundation Trust, London, UK; ${ }^{17}$ Mailman School of Public Health, Columbia University, New York, NY, USA; ${ }^{18}$ Division of Human Development, School of Clinical Sciences, University of Nottingham, Nottingham, UK; ${ }^{19}$ Helmholtz Center Munich, German Research Center for Environmental Health (GmbH), Neuherberg, Germany; ${ }^{20}$ Institute and Outpatient Clinic for Occupational, Social and Environmental Medicine, Clinical Center, Ludwig Maximilians University, Munich, Germany; ${ }^{21}$ IBMC - Institute for Molecular and Cell Biology, and ICBAS, University of Porto, Porto, Portugal; ${ }^{22}$ Department of Audiology, H:S Bispebjerg Hospital and Wilhelm Johannsen Centre of Functional Genomics, University of Copenhagen, Copenhagen, Denmark; ${ }^{23}$ Department of Genetics, University Medical Center Groningen and University of Groningen, Groningen, The Netherlands; ${ }^{24}$ GeneWatch UK, The Mill House, Tideswell, Derbyshire, UK; ${ }^{25}$ PHG Foundation, Worts Causeway, Cambridge, UK

${ }^{*}$ Correspondence: Professor MC Cornel, Department of Clinical Genetics and EMGO Institute for Health and Care Research, VU University Medical Center, BS7, D423, PO Box 7057, 1007 MB Amsterdam, The Netherlands. Tel: +31 20 4448910; Fax: +31 20 4448181; E-mail: mc.cornel@vumc.nl

${ }^{26}$ These authors contributed equally to this work.
} 
possible, then researchers and practitioners have to be prepared for the way in which this changes their daily routine. ${ }^{2-8}$

The clinical management of information about frequently occurring DNA variants that lead to moderate increases in risks for common diseases requires a different approach from that of the significantly increased genetic risks for numerous rare health problems. Translation of research findings to useful health-care applications appears to be lagging behind. Implementation of useful research findings may take years or decades. Meanwhile, some applications of very limited clinical utility have become available directly to the consumers. Difficulties with the translation of research findings need to be understood and addressed if genetics and genomics research is to fulfil its promises towards improving diagnosis, treatment, and prevention. Currently (in 2010) the genetics research community is skeptical about the possibilities of genetic susceptibility testing and screening contributing significantly to the improvement of the quality of health care. The implementation in health care of genetic tests that are considered useful should overcome several thresholds.

Health promotion and disease prevention for the population at large has been the domain of public health professionals; yet, public health approaches have thus far not taken into account genetic risk factors and often not even family history. ${ }^{9}$ So far, advice on lifestyle, physical activity, and nutrition has been developed in a one-size-fitsall approach. ${ }^{6}$ The era of genomics presents the promise of personalized prevention and drug treatment, which has been met with enthusiasm by many people, but called into question by others. ${ }^{10}$

In the light of these new developments in research, there is a pressing need to assess the possibilities for and implications of genetic testing and screening in common diseases (pertaining to multifactorial disorders as well as monogenic subtypes) from both a clinical and a societal perspective. As with genetic testing in rare mendelian disorders, these assessments should comprise analytic validity, clinical validity, clinical utility, and ethical, legal and social issues, ${ }^{11,12}$ as well as health economic aspects. Should a genetic test for a common disease have sufficiently high clinical utility in a specific setting, and should implementation in health care be potentially worthwhile, then the framework for its implementation has to be determined: clinical genetics, medical specialist care, primary care, as a genetic screening program, or as a commercial offer. Currently, as far as common disorders are concerned, testing for monogenic subtypes has mainly been implemented in health care.

In Europe, a shared understanding of and opinion about these developments needs to be established among human and clinical geneticists to enable them to inform future policy making by the European Union (EU) and member states. For this purpose, information was gathered in a background document as input for an expert workshop in Seville, 8-10 October 2007, on 'Clinical validity and utility of genetic susceptibility testing in common disorders'.

In reflecting on the shift in the field of research from rare monogenic to common disorders with a genetic component, this document is a follow-up on several documents and initiatives that have aimed at documenting and harmonizing genetic testing services in Europe. ${ }^{13-20}$ Because of shared interests and complementary expertise, it is a collaborative initiative of three parties: the Public and Professional Policy Committee (PPPC) of the European Society of Human Genetics (ESHG), EuroGentest, and the Institute for Prospective Technological Studies (IPTS). The PPPC is involved in setting professional standards for human and clinical genetics and in issuing recommendations for national and European policy regarding genetic services. EuroGentest is a EU-funded Network of Excellence (NoE) that aims at the standardization and harmonization of genetic testing services and at improving the overall quality of genetic services offered within the EU. This includes both the establishment of procedures and guidelines for the validation of methods and technologies and the provision of quality-assured information sources to medical professionals, as well as proper utilization of genetic services. The IPTS is one of the seven scientific institutes of the European Commission's Joint Research Centre (JRC). It informs EU policy making on issues with a socioeconomic and a scientific or technological dimension.

After the Seville workshop, to enable further discussion on some genetic epidemiological issues, a workshop was organized in September 2008 in Amsterdam. The background document was revised into its present form on the basis of comments by participants of both meetings and other experts, some of whom are active in the EUfunded Public Health Genomic European Network (PHGEN, http:// www.phgen.eu) project and the international GRAPH-Int (http:// www.graphint.org) consortium that was established under the Canadian Public Health Agency. These organizations have been established to promote, at both the European and the global level, responsible and effective translation of genome-based knowledge and technologies into public policy and public health services.

Suggestions were incorporated in the background document, and during the process the PPPC discussed the recommendations. A draft of the background document and recommendations were distributed and posted on the web during the summer of 2009 to elicit further comments. After this procedure the draft was revised. The PPPC and the Board of the ESHG approved the final version. This final text is expected to reflect the views of the European human genetics scientific and professional community.

\section{Scope and limitations}

In this document we will discuss genetic testing and common disorders from a health-care perspective. New possibilities for genetic testing confront health-care workers with the question of whom to test and which test to use. This document focuses on genetic testing and screening in common disorders. The term 'common disorder' is used for disorders that individually have a high impact on public health. Examples of common disorders include cardiovascular disease (CVD), stroke, diabetes, cancer, dementia, and depression. For a health-care practitioner - unlike a geneticist or an epidemiologist - it may not be clear whether a common disorder is due to one gene with a high risk of serious disease, or due to a combination of several genes and several environmental factors. For disorders in which both etiologies exist, both monogenic and multifactorial, the etiology can be thought of as 'complex'. Mendelian diseases can have a complex etiology too: one major gene, many modifying genes, and a series of environmental factors. This is not what we mean by 'complex' in the present document.

Common complex diseases are at present being redefined as a series of diseases with similar symptoms but variable etiology: a few genes, many genes, interactions between genes, many environmental factors, etc. The main difference between 'complex' and 'monogenic or Mendelian' is that in a Mendelian or monogenic disorder an alteration in one gene is a prerequisite for passing the threshold to develop the disorder, although the effect of the mutation could, in some cases, be modulated by genetic variants in other genes or epigenetic events caused by environment, life style, etc, while in complex disorders both whether one will develop disease and the severity are modulated by the complex etiology. In Mendelian diseases one crosses the threshold to disease due to one gene; in complex diseases different factors are needed for crossing the threshold. Between these two extremes there are a series of combinations, for example, the BRCA1 or BRCA2 genes, wherein carrying a mutation will bring an individual close to the 
threshold but other genes and/or environmental factors to push one over it, that is, to cause disease (cancer in this case). Mendelian diseases can be used as an example for common complex diseases, to generate insights into etiological pathways and into avenues for the potential implementation of specific programs in health care. For common complex disorders, genetics and genomics research promises to personalize medicine. Instead of a one-size-fits-all approach, the disorders may be stratified to etiologically different subgroups that also differ according to prognosis and response to treatment and prevention. Lessons learned from rare monogenic conditions may help tailor health care for common complex disorders. Personalizing or tailoring health care will in practice often lead to stratified health care, distinguishing between several groups, while treatment on an individual level is rare.

Different definitions of 'genetic', 'screening', and 'testing' exist. For the purpose of this document we will use genetic (susceptibility) 'testing' in a broad sense, while we use genetic (susceptibility) 'screening' for a systematic, proactive offer to members of a certain group of individuals. Screening may be a well-organized public health program, usually aiming at a low risk population. 'Genetic' testing will be defined as the analysis of DNA or biomarkers for the evaluation of one or more genetic risk factors for a particular disease or disease group.

In health care, genetic screening has to be distinguished from genetic testing because the setting and implications are different. Genetic testing traditionally is carried out on patients or family members who, for whatever reason, have taken the initiative to seek professional advice. Thus, in a health-care setting, testing may provide a health professional with relevant information for diagnosis, prognosis, disease management, and/or further treatment of an individual patient. Genetic testing is also sometimes used in a broader, more comprehensive sense. Genetic tests may be used in genetic screening programs.

Genetic screening traditionally refers to explicit and systematic programs directed either at whole populations of asymptomatic individuals or at subpopulations in which risk is known to be increased or in which the specific phase of life merits screening, such as in the case of pregnant women or newborns. It may be relevant to distinguish between different types of target groups and, furthermore, to distinguish between this systematic approach (eg, as in public health programs) and the situation in which asymptomatic (or lowrisk) persons are offered a test (eg, on the internet). Just as in screening, the recent possibilities for direct-to-consumer genetic testing may be used by asymptomatic persons. Although these tests may be chosen for recreational purposes (such as ancestry testing), they may yield (sometimes unsought) medically relevant information. Commercial genetic testing or screening may be aimed at a broad range of disorders, ranging from traits and common disorders to rare and serious disorders.

In some cases, however, also in the regular health-care setting of a doctor-patient relationship, a more systematic offering of tests may be introduced. This is the case when testing becomes a standard regime for a certain patient group. Testing for mutations in breast cancer tumor tissue provides a good example of such a systematic approach, which can therefore be regarded as a form of genetic screening. In this document, we will indicate specifically which aspects of screening (such as low-risk group, systematic approach) are implied when we refer to screening.

In genetic screening, tests may be offered to individuals by a healthcare agency or a physician, which may give the public the impression that these tests are imposed upon people. The ethical dilemmas are magnified and the responsibilities of the physicians are correspondingly greater. ${ }^{21}$ The genetic nature of a disorder can result in risk implications for the blood relatives of the screened person, even though they may not have been included in the screening programs, nor perhaps wish to be included.

When Wilson and Jungner ${ }^{22}$ formulated their classic list of screening criteria for the World Health Organisation (WHO) in 1968, the possibility of offering treatment was deemed to be an essential prerequisite for offering a sound screening program. Since then, many refinements of these criteria have been formulated, for instance, as regards treatability. Especially in the case of genetic screening, an added aim may be to offer information and options regarding reproductive choices, reducing risk by preventive measures such as changing health behavior, or planning of life events.

Genetic screening for common complex disorders would ideally offer options concerning preventive strategies related to lifestyle, medication, or interventions such as regular monitoring (for example, of biochemical markers in serum, such as cholesterol levels, monitoring of organ function, detecting early premalignant changes, etc). However, the discriminatory power of genetic screening to identify who should or should not be offered particular lifestyle advice or medical interventions remains disputed, especially for 'susceptibility genes' that confer a low relative risk and have a low predictive value.

When using the term 'susceptibility' testing or screening, it can be debated what level of risk might be implied. ${ }^{23}$ The definition in the previous section does not refer to a specific level of risk. A clear-cut distinction between susceptibility testing (indicating a moderately increased risk) and predictive or presymptomatic testing (indicating a severely increased risk) cannot be given. In principle, testing for relatively high penetrant genes, such as $B R C A 1$ or $B R C A 2$ (indicating a lifetime risk of developing hereditary breast cancer of between 60 to $85 \%$ ), could be regarded as a form of susceptibility testing, and, most notably, in the United States the term 'susceptibility testing' is also used for these highly penetrant disorders. In practice, however, those kinds of informative tests are considered to be on the predictive side, pertaining to highly penetrant monogenic disorders and monogenic subforms of common disorders, whereas the term 'susceptibility testing' is reserved for relatively lower predictive values associated with common disorders.

This document reviews and discusses current biomedical, epidemiological, ethical, social scientific, public health, health economic, and health technology assessment (HTA) literature on genetic testing and screening in common disorders, as well as documentation on regulatory and policy issues.

A search was performed in PubMed-medline and the Social Science Citation Index using keywords relevant to genetic susceptibility and testing or screening for common disorders. In addition, references from relevant articles were selected. Especially reviews, reports, position papers, and editorials were considered. Reports on HTAs concerning genetic susceptibility testing were also used. For this purpose, several databases accessible via the internet were surveyed and the relevant literature on the topic of (economic) evaluation of testing services was reviewed (see Appendix C).

Given the two poles of rare, often severe, monogenic disorders caused by highly penetrant genotypes on the one hand and polygenic, multifactorial (complex) common disorders associated with genotypes of low penetrance on the other hand, this document focuses upon the common disorders associated with genotypes of higher or low penetrance: in particular, those conditions where the public health implications need to be considered - that is, at which level of clinical utility tests should be made available and how they should be implemented in practice. For many common disorders, monogenic subtypes exist, including certain forms of cancer (most notably colon 
cancer and breast and ovarian cancer). It appears worthwhile exploring to what extent health-care practices developed for these disorders may help identify challenges for the future implementation of knowledge of the human genome into health care. ${ }^{24}$ Learning from health care for monogenic disorders and monogenic subtypes may help to decide on potential avenues of implementation or non-implementation in health care.

A limitation of this document is the absence or scarcity of information on molecular pathways, population genetic data, test performance, and social, psychological, ethical and health economic data, and experience regarding susceptibility testing. Furthermore, the settings in which tests are piloted or marketed are constantly changing. As Calnan et $a l^{25}$ note: 'New technologies rarely emerge fully formed at their optimum effectiveness and lowest cost; rather they develop in situation, as clinicians master new techniques and the existing technology is tweaked and modified to suit the clinical context...the cost of a new technology may fall rapidly as development costs are covered, uptake rates increase, and the healthcare provider reorganises to provide the service more efficiently. All of this happens a considerable way down the implementation pathway.' Therefore, the data we could obtain for this background document may prove to be of limited value. However, science progresses rapidly, and genome-wide association studies are revealing associations between common disorders and genetic variants at a fast pace, which urges a common understanding of, and vision for, the possibilities of genetic testing and screening in common complex disorders.

For this reason, we will make use of analogies with existing forms of testing and screening, including testing and screening for monogenic subtypes of common complex disorders, whenever this seems appropriate. Special cases such as hemochromatosis (monogenic, yet not highly penetrant) and familial hypercholesterolemia (FH; monogenic, highly prevalent, but only one out of many risk factors for CVD) will also be discussed. The health care provided for these disorders in various countries and health centers falls outside the scope of clinical genetics, and could guide our thinking about future genetic testing and screening in common complex disorders.

This document will not consider germline prenatal or preconceptional testing, nor testing of biomarkers for tumor recurrence, but it will discuss testing of mutations in tumor tissue, since this may reveal susceptibility to certain forms of therapy. Also, pharmacogenomic applications will not be discussed in depth, although some examples will be given of pharmacogenomic testing.

Outline. In the next section, the terrain of common complex disorders is introduced. Different assessment frames for genetic testing and screening are discussed. The section following that examines the aims and strategies for genetic testing and screening in common disorders and discusses some examples of current testing and screening in Europe. The section 'The economic evaluation of genetic tests' discusses the cost-benefit relation of different types of tests and screening strategies and how they could be used in the clinic in a cost-effective way. The subsequent section addresses the ethical, legal, and social issues of testing and screening in common disorders. The last section addresses regulatory and intellectual property issues in the $\mathrm{EU}$ as well as the United States.

\section{COMMON DISEASES}

In the second half of the last century, medical genetics was mainly occupied with rare chromosomal disorders and monogenic conditions, including inborn errors of metabolism, dysmorphic, and mental retardation syndromes. The challenge in common complex diseases, as recognized by clinical geneticists, was mainly to identify subtypes of the diseases that were due to single gene mutations of high penetrance, and to offer affected families the genetic services they need. ${ }^{16,17}$ While the recent EuroGentest document on genetic testing services in Europe $^{13}$ concentrated on hereditary, mostly rare disorders, and on the dominant role of clinical geneticists in their management, the present documentation is concerned with the clinical validity and utility of genetic tests for common complex disorders (both multifactorial and monogenic subtypes). When used either in the setting of an individual doctor-patient relationship or in that of systematic screening, these tests would interface with everyday medical practice, impacting on the majority of clinical specialties and primary care.

In 2003, the ESHG stated that 'beyond the rare Mendelian subsets, genetic influences in common diseases are likely to be conditional on the environment. Testing for these low penetrance susceptibility genes is likely to be of limited clinical utility?. However, as is illustrated by Figure 1, a clear distinction between Mendelian and 'the remainder' is not possible. Firstly, most monogenic disorders are also affected by modifier genes and environmental factors, and therefore are not as simply 'Mendelian' as initially thought. For instance, for narcolepsy the genetic factor HLADR2 antigen is necessary, but very few individuals with that variant develop the disease. ${ }^{26}$ The same holds for HLA-B27 carriers and ankylosing spondylitis. ${ }^{27}$ Some monogenic disorders may only manifest in a specific environment; for instance, the metabolic disorder phenylketonuria (PKU) will only cause significant disease in the presence of a protein-containing diet, and in G6PD deficiency hemolytic anemia occurs only if the affected individuals eat fava beans or take sulfonamides. In other instances, the clinical picture of a monogenic disorder depends upon environmental influences, such as bacterial (Pseudomonas aeruginosa) infections in patients with CF. In most common diseases, the clinical manifestation of any particular genetic factor is likely to be precipitated or modified, to a variable extent, by other genetic or environmental factors. Bearing this in mind, Figure 1 can help to understand different etiological contributions of genetic and environmental factors. It should not be regarded as a linear model.

Most, if not all, common diseases are etiologically heterogeneous: different cases can be caused by different etiological factors. Often, numerous genetic variants and multiple environmental factors have a role. ${ }^{29-31}$ Different combinations of genetic factors and combinations of genetic and environmental factors may be involved in the disease history of different patients. In addition, rare major genes may be

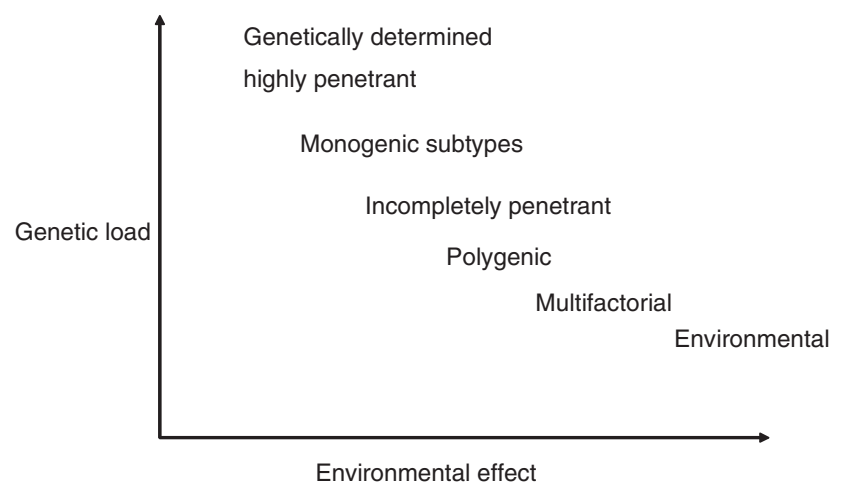

Figure 1 The genetic etiology of disease, a spectrum from 'genetically determined' to 'environmental'.

Source: Modified from Bomprezzi et al. ${ }^{28}$ Adapted by permission from BMJ Publishing Group Limited. Copyright (C) 2003. 
Table 1 Examples of common disorders and their heritability

\begin{tabular}{lc}
\hline Multifactorial disease & Heritability \\
\hline Age-related maculopathy & 0.45 \\
Age-related macular degeneration & $0.46-0.71$ \\
Crohn disease & 1.0 \\
Prostate cancer & 0.42 \\
Breast cancer & 0.27 \\
Type 2 diabetes & 0.26 \\
Obesity & $0.40-0.54$ \\
Coronary artery disease (CAD) & 0.49 \\
Death from CAD & $0.57^{\mathrm{a}}$ \\
& $0.38^{\mathrm{b}}$ \\
Hypertension & 0.80 \\
\hline aMales. & \\
bFemales. & \\
Source: Modified from Lango and Weedon. ${ }^{36}$ Adapted by permission from John Wiley $\&$ Sons, \\
Inc. Copyright $\mathbb{C}$ 2008 (http://onlinelibrary.wiley.com/journal/10.1111/(ISSN) 1365-2796).
\end{tabular}

relevant. Research into the genetic basis of common diseases is increasingly focusing on all genes, not only on those causing Mendelian subforms. Approaches are becoming increasingly large-scale, and hypothesis-driven studies are being replaced by hypothesis-generating ones, such as genome-wide association studies. Instead of assessing single-gene effects, future work will concentrate upon characterizing complex and interacting pathways by means of systems biology. In addition to the human (host) genome, the genomes of pathogens (such as the malaria parasite), vectors (such as the Anopheles gambiae mosquito), or model organisms (such as the mouse) are also being studied.

Mortality and morbidity related to common diseases have a high public health impact. Most deaths and hospital admissions in adults are related to common diseases, and only few to rare diseases. The medical specialties involved in common diseases are very diverse (oncology, cardiology, rheumatology, etc). While clinical geneticists, molecular geneticists, and cytogeneticists still focus mainly on the domain of rare disorders, caused by highly penetrant mutations, their role in the genetics of complex diseases is less clear. For most clinicians, the genome era has not yet arrived. ${ }^{32}$ For genomics research to also have an effect on clinical practice, health-care providers need to become more literate in genomics. Building bridges between basic researchers, genetic experts, and health-care professionals is therefore of paramount importance.

Heritability reflects the proportion of inter-individual variation in disease susceptibility in a population that is estimated to be due to genetic variation. It is therefore a crude measure of the genetic component of disease etiology. Heritability varies in different populations and is dependent on the assumptions made in the calculations, which have been questioned by some authors. ${ }^{33-35}$ A heritability of 0.5 suggests that half of the variation in disease susceptibility in the population may be explained by genetic variation. Heritability estimates for some common diseases are given in Table 1. Owing to formal and experimental imponderabilities, however, most of these values should not be regarded as absolute.

For practical purposes, it may be useful to categorize common diseases on the basis of organ manifestation or pathogenesis. The International Classification of Diseases ${ }^{37}$ takes this approach, and many HTA studies therefore start from disease categories such as 'CVD' (based on the organ system) or 'cancer' (based on the pathogenesis).

In nearly all common disease categories, one or more subgroups can be identified - comprising at most $5-10 \%$ of all cases in the
Table 2 Examples of common diseases with Mendelian subsets

\begin{tabular}{|c|c|}
\hline Common disease & Mendelian subsets \\
\hline Atherosclerosis & $\begin{array}{l}\text { Familial hypercholesterolemia due to low-density lipoprotein } \\
\text { (LdL) receptor mutations }\end{array}$ \\
\hline $\begin{array}{l}\text { Cirrhotic liver } \\
\text { disease }\end{array}$ & Hemochromatosis due to HLA-H gene mutations \\
\hline Emphysema & $\alpha_{1}$-antitrypsin deficiency \\
\hline Diabetes & $\begin{array}{l}\text { Maturity-onset diabetes of the young (MODY) due to mutations in } \\
\text { (a) MODY } 1 \text { (hepatocyte nuclear factor-4a gene), } \\
\text { (b) MODY } 2 \text { (glucokinase gene) } \\
\text { (c) MODY } 3 \text { (hepatocyte nuclear factor-1a gene) }\end{array}$ \\
\hline Breast cancer & $\begin{array}{l}\text { Autosomal dominant hereditary breast cancer due to mutations in } \\
\text { (a) BRCA1 } \\
\text { (b) BRCA2 } \\
\text { (c) P53 } \\
\text { (d) PTEN }\end{array}$ \\
\hline Colon cancer & $\begin{array}{l}\text { (a) Familial adenomatous polyposis coli (FAP) due to APC } \\
\text { mutations } \\
\text { (b) Hereditary non-polyposis coli (HNPCC) due to mismatch } \\
\text { repair gene mutations }\end{array}$ \\
\hline $\begin{array}{l}\text { Alzheimer's } \\
\text { disease }\end{array}$ & $\begin{array}{l}\text { Autosomal dominant Alzheimer's disease due to mutations in } \\
\text { (a) APP (amyloid precursor protein gene) } \\
\text { (b) PS1 (Presenilin } 1 \text { gene) } \\
\text { (c) PS2 (Presenilin } 2 \text { gene) }\end{array}$ \\
\hline
\end{tabular}

Source: Rose and Lucassen. ${ }^{38}$ Reproduced by permission from Oxford University Press. Copyright (C) 1999.

population - which appear to be due to the action of single major genes. They comprise the so-called 'Mendelian subforms' of common diseases (see Table 2). However, it is important to bear in mind that these rare, major gene mutations may have variable penetrance.

Evaluations of the clinical validity and utility of genetic testing and screening for these subsets should follow the same rules as recommended for other Mendelian disorders. ${ }^{13}$ It should be borne in mind, however, that the great majority of patients and families falling into the Mendelian subgroup category of common disorders have not yet been identified and properly dealt with. ${ }^{39}$ Recognizing this subset of cases, for which meaningful genetic testing is indeed available and is of proven utility, should be an important goal of public health genetics education and practice.

\section{Genetic contribution to disease etiology}

The spectrum of diseases, ranging from genetically determined to environmental (see Figure 1), comprises several etiological categories ${ }^{40,41}$ such as, for example:

- monogenic (Mendelian): disease-causing single-gene mutation;

- chromosomal: numerical or morphological abnormalities, causing disabilities of varying extent;

- multifactorial: combined effects of multiple genetic variants and their interaction with a range of environmental factors (including infectious diseases, for which the susceptibility, clinical course, or effectivity of treatment may be influenced by genetic factors);

- exogenous or environmental.

'Multifactorial' and 'complex' etiologies are not completely synonymous. A disorder with a complex etiology may be both heterogeneous in etiology (including monogenic subgroups or cases with a purely 
exogenous etiology) and (usually) have a multifactorial etiology where both genes and environmental factors are involved.

When comparing Mendelian with multifactorial disorders, the former are characterized by a relatively deterministic genotype-phenotype relationship, while the latter show a vast complexity resulting in incomplete penetrance. For example, carriership of the Factor $\mathrm{V}$ Leiden (FVL) mutation increases the risk for venous thromboembolism (VTE) to an estimated $10-20 \%$ in a lifetime, but the genotype is neither sufficient nor necessary for VTE to occur. ${ }^{42}$

Genetic components of multifactorial traits can affect the susceptibility to disease (clinical/pre-clinical), underlie differences in the natural history of disease (severity, complications, prognosis), or result in different therapeutic responses (efficacy, adverse effects). ${ }^{43}$

\section{Gene-disease associations}

Estimates on the frequency of multifactorial diseases indicate that, on average, more than 46 out of 1000 newborn children in industrialized countries will manifest such a disease, with a genetic component, by the age of 25, and 600 individuals (out of 1000) after the age of 25, which implies a lifetime risk of almost $65 \%{ }^{44}$ This implies that the vast majority of people will be affected by one or more complex diseases. According to many authors, these are presumed to result from the cumulative effect of mutations of low penetrance at multiple loci (ie, susceptibility genes) which, if occurring in isolation, would not have any phenotypic effect. In combination and in the presence of the necessary environmental influences and/or lifestyle factors, these mutations increase the risk of disease. ${ }^{45}$ To identify causal mutations, variations in the genome have been studied that may be closely linked to the disease-causing alleles. For a specific individual, heterozygosity at any given site will in most instance be due to one of the common single-nucleotide polymorphisms (SNPs), ${ }^{46}$ defined by a frequency of both its alleles of more than $1 \%$. Either directly or through linkage disequilibrium, these variants may contribute significantly to the genetic risk for common complex diseases. The literature contains numerous studies of associations between common DNA polymorphisms and variation in disease susceptibility, and since 2007 many more association studies have been published.,47-49

However, many association studies in the past have been small and of limited validity. Strong associations found in small studies were typically not confirmed by larger studies that followed, and it has become evident that initial association studies tend to overestimate the genetic predisposition to common diseases. ${ }^{2,36,50}$

Recently, genome-wide association studies ${ }^{3}$ and meta-analyses ${ }^{49}$ have yielded more and more confirmed associations between genetic variants and common disease. Yet, even the large-scale genome-wide association study by the Wellcome Trust Case Control Consortium arrived at the conclusion that the predictive value of known polymorphisms will be limited and will therefore be unlikely to trigger preventive decisions or actions. ${ }^{3}$

The common disease-common variant hypothesis - proposing that the genetic risk for common disease is based on a number of predisposing variants in susceptible populations - appears unlikely to be correct. ${ }^{29-31,51}$ However, some argue that it might well be possible that both common and rare alleles are important. ${ }^{36}$

The clinical validity of a genetic test is determined by the association between the genetic variant to be tested and the disease in question, measured by sensitivity, specificity, and likelihood ratios, and the prevalence of the disease, which impacts upon the positive and negative predictive values.

Recent publications suggest that claims of clinical validity for common polymorphisms are often premature ${ }^{52-54}$ because most, if not all, associations are only of small or modest size (ie, OR $<1.4$; see Table 3 ).

Table 3 Findings of recent whole-genome association studies

\begin{tabular}{|c|c|c|c|c|c|c|}
\hline Disease & Source & Gene/locus & $O R_{\text {het }}$ & $O R_{\text {hom }}$ & $\mathrm{P}$ value & PAR (\%) \\
\hline \multirow[t]{8}{*}{ Breast cancer } & Easton et $a l^{55}$ & FGFR2 & 1.20 & 1.64 & $1 \times 10^{-10}$ & 16 \\
\hline & \multirow[t]{5}{*}{ Hunter et al ${ }^{56}$} & FGFR2 & 1.23 & 1.63 & $2 \times 10^{-76}$ & - \\
\hline & & TNRC9 & 1.23 & 1.39 & $1 \times 10^{-36}$ & - \\
\hline & & MAP3K1 & 1.13 & 1.27 & $2 \times 10^{-20}$ & - \\
\hline & & $L S P 1$ & 1.06 & 1.17 & $3 \times 10^{-9}$ & - \\
\hline & & $8 q 24$ & 1.06 & 1.18 & $5 \times 10^{-12}$ & - \\
\hline & \multirow[t]{2}{*}{ Stacey et al ${ }^{57}$} & $2 q 35$ & 1.11 & 1.44 & $5 \times 10^{-14}$ & 14 \\
\hline & & TNRC9 & 1.27 & 1.64 & $6 \times 10^{-19}$ & 13 \\
\hline Coronary artery disease & McPherson et al ${ }^{58}$ & 9p21 & 1.20 & - & $4 \times 10^{-6}$ & 13 \\
\hline Myocardial infarction & Helgadottir et al ${ }^{59}$ & 9p21 & 1.25 & 1.64 & $1 \times 10^{-20}$ & 21 \\
\hline Obesity & Frayling et al 60 & FTO & 1.32 & 1.67 & $3 \times 10^{-35}$ & 20 \\
\hline \multirow[t]{10}{*}{ Diabetes } & \multirow[t]{4}{*}{ Sladek et $a l^{61}$} & TCF7L2 & 1.65 & 2.77 & $1 \times 10^{-34}$ & 28 \\
\hline & & SLC30A8 & 1.18 & 1.53 & $6 \times 10^{-8}$ & 24 \\
\hline & & HHEX & 1.19 & 1.44 & $3 \times 10^{-6}$ & 19 \\
\hline & & EXT2 & 1.25 & 1.50 & $1 \times 10^{-4}$ & 16 \\
\hline & Steinthorsdottir et al 62 & CDKALI & 1.25 & 1.50 & $8 \times 10^{-9}$ & 16 \\
\hline & \multirow[t]{3}{*}{ Scott et al ${ }^{63}$} & $\mid G F 2 B P 1$ & 1.14 & - & $9 \times 10^{-16}$ & - \\
\hline & & $C D K N 2 A / B$ & 1.20 & - & $8 \times 10^{-15}$ & - \\
\hline & & $11 \mathrm{p} 12$ & 1.25 & - & $4 \times 10^{-7}$ & - \\
\hline & \multirow[t]{2}{*}{ Zeggini et al ${ }^{64}$} & KCNJ11 & 1.14 & - & $5 \times 10^{-11}$ & - \\
\hline & & PPARG & 1.14 & - & $2 \times 10^{-14}$ & - \\
\hline \multirow[t]{2}{*}{ Prostate cancer } & Yeager et $a l^{65}$ & $8 q 24$ & 1.26 & 1.58 & $9 \times 10^{-13}$ & 21 \\
\hline & Gudmundsson et al 66 & $8 q 24$ & 1.71 & - & $2 \times 10^{-14}$ & 13 \\
\hline
\end{tabular}

Abbreviations: $\mathrm{OR}_{\text {het, }}$ odds ratio - heterozygotes; $\mathrm{OR}_{\text {hom }}$, odds ratio - homozygotes; '-', not reported; PAR, population-attributable risk.

Source: Modified from Topol et al. ${ }^{4}$ Copyright (C) 2007 American Medical Association. All rights reserved. 
Any precise estimation of disease risks would require numerous genetic variants to be considered in combination with environmental factors. As known genetic variants explain only a small proportion of the estimated heritability of common diseases, current risk estimates are not stable and the correlation between the predicted and actual genetic risk is likely to be poor. ${ }^{30}$ Moreover, not all variants are known and testable, and knowledge about gene-gene and geneenvironment interactions is often lacking. ${ }^{67}$ While some authors argue that discovery of these missing variants may allow useful predictions of genetic susceptibility to be made in future ${ }^{68}$, others are more skeptical. $^{69-73}$

It should be acknowledged that, in understanding the genetic susceptibility for many common complex disorders, we are only at the beginning of finding the pieces of a puzzle of unknown size and shape. Some people doubt the usefulness of this line of research. ${ }^{74}$ In addition, for many disorders, little is known about effective interventions - such as taking medication or altering the lifestyle - to prevent disease or postpone its onset. In most cases of rare polymorphic variants, the change in risk would be too small for any public health intervention to be appropriate. For most diseases, the number of known susceptibility SNPs would be too small for a polygenic test, but in some cases a combination of risk variants may confer a sufficiently high relative risk to support the recommendation of risk-reducing strategies, such as regular monitoring. ${ }^{75}$ In public health, more examples can be given of the use of moderate (non-genetic) risk factors, such as age, blood pressure, BMI, and waist circumference.

Currently, family history may often be more predictive than genetic variants, which may only alter disease susceptibility by a factor of 1.2 each, or less. Familial clusters (recognised among close relatives of affected individuals) may identify subgroups of a population at increased risk that can form the target for further specialized actions.

Regarding the usefulness of genetic testing or even screening in common disorders, knowledge of the gene-environment interaction is argued to be paramount. In case of known gene-environment interactions for certain gene variants, people carrying that variant may profit the most. ${ }^{76}$ However, for fixed risk factors, that is, when the intervention does not change the risk factor itself, the risk-benefit ratio of an intervention may be lower (or no different) when targeted at a high-risk group, than for members of the general population. This means that those who are at highest genetic risk may or may not be those who gain most from an intervention. It should be noted that the contribution of genetic factors to some common multifactorial diseases are fixed risks and cannot be changed by any intervention. $^{35}$

Owing to often greatly reduced penetrance, individuals carrying the 'high-risk' form of a gene may not develop the disorder in question, while individuals without the genetic predisposition may show characteristics of the disease, possibly caused by a single environmental exposure (eg, smoking, exercise, nutrition). ${ }^{36,77}$ A precise and comprehensive risk assessment is not possible merely on the basis of genetic variants. If a genetic susceptibility test was used to advise a subgroup of the population (at high environmental risk) to change their lifestyle (ie, the environmental risk factor), a proportion of cases could be avoided. The utility of the test depends on whether or not a (positive) gene-environment interaction exists. If no interaction exists, the implementation of a screening program would not automatically improve the population health compared with randomly selecting the same number of people, and there might even be negative health benefits because of the screening. For this reason, any assessment of the likely effects on health caused by genetic tests combined with environmental or lifestyle advice would be greatly facilitated

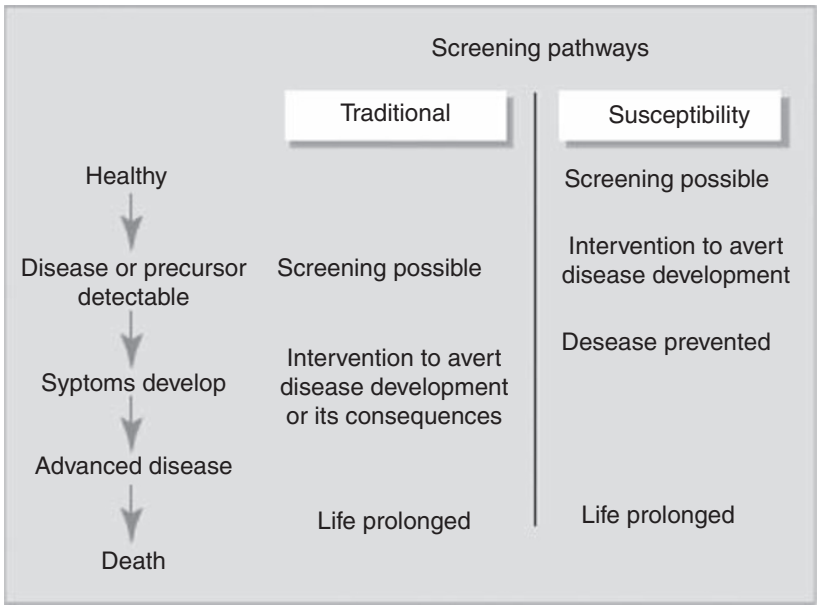

Figure 2 Potential screening pathways.

Source: Reproduced from Goel. ${ }^{80}$ Copyright (C) 2001 with permission from BMJ Publishing Group Ltd.

knowledge of the magnitude (and sign) of any gene-environment interaction. ${ }^{35,78,79}$ It can be argued, however, that genetic testing may be of clinical utility even in the absence of gene-environment interaction if taking the test and having knowledge about risk status supports changes in behavior and life style. As changing habits and health behavior has proven to be difficult, in some cases genetic testing might give individuals an extra stimulus. However, currently, research studying the impact of susceptibility testing on changing behavior is limited.

Given these provisos, which should not be underestimated, confirmed gene-disease associations might perhaps become of importance for the prediction, prevention, and treatment of common diseases in the future. Especially the use of genetic susceptibility screening in asymptomatic individuals would represent a chance for early identification of individuals at increased risk of a specific condition. This could lead to reduced morbidity and mortality if interventions are available to prevent or postpone the onset of disease (see Figure 2).

In the wording of Lango and Weedon, ${ }^{36}$ ' .... we finally have the ability to systematically identify the genetic variants underlying a range of common disorders from type 2 diabetes to Crohn's disease, but when will these findings benefit clinical medicine, and are we on the right track to using this genetic information to improve clinical practice? It should be remembered that this type of genetic research is still in its infancy, and as we have only just acquired the ability, through GWA studies, to identify a large number of common disease genes, it is unsurprising that there has been no major impact of this research on clinical practice as yet. However, the ability of genetic research to alter treatment and diagnosis, given sufficient time, is clearly demonstrated by the success in the monogenic field, for example, in Maturity-Onset Diabetes of the Young (MODY). We expect there will be a great benefit to clinical medicine in the years to come, and while prediction of disease and pharmacogenetics may eventually prove valuable, the greatest clinical benefit of GWA studies is likely to come from etiological insights into disease processes.' However, while most authors recognize that genetic research can provide insights into disease processes, others are skeptical about disease prediction. For example, Clayton ${ }^{69}$ argues that individual disease prediction is an ambitious aim and that even if all the relevant genetic variants, including rare variants, were to be discovered, the combined effect will have modest predictive power for most diseases. 


\section{Assessment of genetic screening and testing}

Elaborate frameworks have been developed for the assessment of genetic screening programs directed at specific groups or the population at large, as well as for the assessment of tests used in genetic services. The evaluation of genetic screening programs has to include evaluation of the test characteristics, complemented by additional considerations regarding the screening context (ie, purpose, targeted groups).

Criteria for the evaluation of screening programs. One potential of genetic testing services for predictive and preventive medicine arises from the possibility of identifying individuals at risk. In order to target interventions to individual patients and to improve their health and prevent disease, the screening of populations or specific subgroups provides the possibility to determine individual susceptibility to common multifactorial disorders. Screening is targeted at populations that are not aware of potentially being at increased risk and have not sought medical attention for the condition in question. The purpose is to identify persons at increased risk of a specific disorder (whereupon further testing can be done, or preventive actions taken) and to offer follow-up and intervention to benefit the screened individuals. In 1968, Wilson and Jungner developed principles of population screening that can also be applied in the case of disorders with a genetic component (see Box 1). Based on the criteria by Wilson and Jungner, ${ }^{22}$ the Crossroads 99 Group devised a framework to assess susceptibility testing for breast, ovarian, and colorectal cancer. ${ }^{80}$ The Crossroads Criteria are based on a simple model of disease progression (see Figure 2), which indicates that screening tests primarily detect genetic susceptibility to disease at a preclinical, asymptomatic phase. The
Wilson and Jungner criteria and the domains of these criteria were broadened to some extent. For example, the first domain, Knowledge of disease, was extended to include knowledge of the population; the criterion of knowledge about the developmental stages of a disorder was extended so as to include knowledge about risk and susceptibility. The criterion that a treatment should be available was diverted into the notion that interventions that have physical, psychological, and social net benefit should be available. Cost considerations within the Crossroads Criteria refer to the broader field of societal and health system issues. Overall, much more attention is paid to ethical, legal, and social issues. Through these modifications the usefulness of the original criteria by Wilson and Jungner was confirmed, while, at the same time, developments in the field of genetic susceptibility testing were acknowledged.

In 2001, Burke et $a l^{81}$ applied the Wilson and Jungner screening criteria to genetic testing or screening in three adult-onset conditions (breast cancer, colorectal cancer, and hemochromatosis). Although the authors concentrated upon the evaluation of major gene effects, their work nevertheless confirmed that each of the Wilson and Jungner criteria can be applied in principle in a genetic testing and screening context. Table 4 gives a general idea of how the criteria by Wilson and Jungner can be applied in detail, although it should be noted that some issues have advanced in recent years (eg, facilities for diagnosis and treatment).

At the same time, Table 4 identifies important knowledge gaps concerning genetic testing for breast and colorectal cancer, as well as screening for hemochromatosis. These gaps result from uncertainties that will be found to be associated with many common diseases. In the case of breast and ovarian cancer, only a minority of cases are

\section{Box 1 Criteria for assessment of screening}

Wilson and Jungner

Knowledge of disease

Condition must be an important problem.

Recognizable latent or early symptomatic stage.

Natural course of condition (including development from latent to declared disease) should be adequately understood.

\section{Knowledge of test}

Suitable test or examination.

Test acceptable to the population.

Case finding should be a continuing process and not

'once and for all' project.

Treatment for disease

Accepted treatment for patients with recognized disease.

Facilities for diagnosis and treatment available.

Agreed on policy concerning whom to treat as patients.

Cost considerations

Costs of case finding (including diagnosis and treatment of patients diagnosed) economically balanced in relation to possible expenditures on medical care as a whole.
Crossroads $99^{\mathrm{a}}$

Knowledge of population and disease

Burden of target disease should be important.

Target population or population at risk identifiable.

Considerable level of risk or latent or preclinical phase.

Natural course (from susceptibility to precursor, early disease, and advanced disease) should be adequately understood.

Feasibility of screening procedures

Suitable test or examination.

Entire screening procedure acceptable to the population.

Screening should be a continuing process and should encompass all elements of screening procedures.

\section{Interventions and follow-up}

Interventions that have physical, psychological, and social net benefit available. Facilities for adequate surveillance, prevention, treatment, education, counselling, and social support available.

Consensus on accepted management for those with positive test results.

Societal and health system issues

Costs should be balanced in economic, psychological, social, and medical terms and with health-care expenditures as a whole.

Appropriate screening services accessible to the entire population, without adverse consequences for non-participants.

Appropriate confidentiality procedures and antidiscrimination provisions

for participants and non-participants.

aEthical, legal, and sociobehavioral issues are considered across all domains. Screening should be considered within a framework that recognizes fundamental human rights. Source: Reproduced from Goel. ${ }^{80}$ Copyright (C) 2001 with permission from BMJ Publishing Group Ltd. 
Table 4 Application of screening principles to genetic screening

\begin{tabular}{|c|c|c|c|}
\hline & $\begin{array}{l}\text { BRCA1/2 mutations } \\
\text { Testing women with family histories } \\
\text { indicating } \geqslant 10 \% \text { chance of mutation }\end{array}$ & Testing people of Ashkenazi descent & General population screening \\
\hline Important health problem & Yes (breast and ovarian cancer) & Yes (colorectal cancer) & Yes (iron overload disease) \\
\hline Accepted and specific treatment & $\begin{array}{l}\text { Effectiveness of interventions to reduce } \\
\text { risk uncertain; prophylactic surgery } \\
\text { unacceptable to many women }\end{array}$ & $\begin{array}{l}\text { Reduced cancer mortality with colorec- } \\
\text { tal screening, but need for genotypic- } \\
\text { specific screening program uncertain }\end{array}$ & Yes (phlebotomy) \\
\hline Facilities for diagnosis and treatment & $\begin{array}{l}\text { Adequacy of testing, counselling, and } \\
\text { follow-up, not evaluated in clinical } \\
\text { or public health settings }\end{array}$ & $\begin{array}{l}\text { Adequacy of testing, counselling, and } \\
\text { follow-up, not evaluated in clinical or } \\
\text { public health settings }\end{array}$ & $\begin{array}{l}\text { Adequacy of testing, counselling, and } \\
\text { follow-up, not evaluated in clinical or } \\
\text { public health settings }\end{array}$ \\
\hline Early stage known & Yes & Yes & Yes \\
\hline Suitable test & Uncertain predictive value & Uncertain predictive value & Uncertain predictive value \\
\hline Test is acceptable & $\begin{array}{l}\text { Uncertainty concerning the health } \\
\text { benefit from screening; social and } \\
\text { psychological risks not known }\end{array}$ & $\begin{array}{l}\text { Uncertainty concerning the health } \\
\text { benefit from screening; social and } \\
\text { psychological risks not known }\end{array}$ & $\begin{array}{l}\text { Uncertainty concerning the health ben- } \\
\text { efit from screening; social and psycho- } \\
\text { logical risks not known }\end{array}$ \\
\hline Natural history known & No & $\begin{array}{l}\text { Probably not different from sporadic } \\
\text { colorectal cancer }\end{array}$ & No \\
\hline Agreement about when to treat & No & No & No \\
\hline Cost of care, funding acceptable & Uncertain & Uncertain & Uncertain \\
\hline Ongoing process & Cannot yet be addressed & Cannot yet be addressed & Cannot yet be addressed \\
\hline
\end{tabular}

Source: Reproduced with permisson from Burke et al. ${ }^{81}$ The publisher for this copyrighted material is Mary Ann Liebert, Inc. publishers.

\section{Box 2 Principles of population screening as applied to genetic susceptibility to disease}

Public health assessment

The disease or condition should be an important public health burden to the target population in terms of illness, disability, and death.

The prevalence of the genetic trait in the target population and the burden of disease attributable to it should be known.

The natural history of the condition, from susceptibility to latent disease to overt disease, should be adequately understood.

Evaluation of tests and interventions

Data should be available on the positive and negative predictive values of the test with respect to a disease or condition in the target population.

The safety and effectiveness of the test and accompanying interventions should be established.

Policy development and screening implementation

Consensus regarding the appropriateness of screening and interventions for people with positive and negative test results should be based on scientific evidence. Screening should be acceptable to the target population.

Facilities should be available for adequate surveillance, prevention, treatment, education, counselling, and social support.

Screening should be a continual process, including pilot programs, evaluation of laboratory quality and health services, evaluation of the effect of screening, and provisions for changes on the basis of new evidence.

The cost effectiveness of screening should be established.

Screening and interventions should be accessible to the target population.

There should be safeguards to ensure that informed consent is obtained and the privacy of those tested is respected, that there is no coercion or manipulation, and that those tested are protected against stigmatization and discrimination.

Source: Khoury et al. ${ }^{82}$ Copyright (C) 2003 Massachusetts Medical Society. All rights reserved.

accounted for by mutations in the highly penetrant genes, and only these can currently be screened for. The rest of the genetic 'load' of both disorders is likely due to a combination of the effects of multiple minor genes interacting with environmental influences.

Regarding the relevance of population screening for genetic susceptibility, Khoury et al ${ }^{82}$ suggested some modifications and refinements to the Wilson and Jungner criteria on the basis of the Crossroads modifications.

The criteria for the evaluation of screening programs provide a framework to assess the benefit of genetic screening by considering different dimensions of a screening program before its widespread implementation (see Boxes 1 and 2). The quality of a test is the most relevant aspect when it comes to its application in a screening program. Before a final decision is made as to whether or not to implement a genetic screening program, the relevant genetic tests have to be evaluated according to their overall performance and effectiveness.

Although the often revised and refined Wilson and Jungner criteria are still frequently referred to, more elaborate frameworks have been developed in recent years that take elements of the setting in which a genetic test is used into consideration (for instance, in a screening program) and combine this with quantitative information about the disorder and the tests to be applied. The ACCE framework is an example of such a framework, quantifying as many criteria as possible (eg, public health burden, quality of a test) and thereby facilitating a 
more detailed and rational assessment of the potential benefits of testing or screening. ${ }^{83}$

The ACCE framework was developed by the Centers for Disease Control and Prevention (CDC, USA), National Office of Public Health and the Foundation of Blood Research. It has been proposed for the assessment of emerging new genetic tests. ${ }^{84}$ The CDC initiated another project, the Evaluation of Genomic Applications in Practice and Prevention (EGAPP), in order to support the development of a systematic process for evaluating genetic tests and other genomic applications that are in transition from research to clinical and public health practice. ${ }^{85}$ So far, the EGAPP has provided evidence reports on several genetic tests (eg, for ovarian cancer, cytochrome 450 polymorphisms, hereditary non-polyposis colorectal cancer, and gene expression profiling tests in case of breast cancer).

Similar approaches have been taken in Canada, the United Kingdom, and Germany, where evaluation processes have started. The United Kingdom Genetic Testing Network Steering Group, which is involved in the commissioning and provision of genetic tests in the UK National Health Service (NHS), has endorsed and adapted the ACCE core principles to produce a 'gene dossier' for more than 150 genetic tests, ${ }^{86}$ most of which are for clinical use. ${ }^{87}$ In Canada, CanGèneTest aims to evaluate the effectiveness of genetic laboratory services in Canada and to develop tools and approaches to help decision makers to establish the relevance of new diagnostic genetic technologies. ${ }^{88}$ The German Society of Human Genetics is currently publishing a series of disease-specific indication criteria for genetic testing. ${ }^{89}$ This activity is based on a preceding Eurogentest workpackage and is now being complemented by the Eurogentest 'clinical utility gene cards.90

Quantitative measures and the ACCE framework. When evaluating genetic tests, many uncertainties will arise. The genetic epidemiology of the condition under consideration has to be considered within the scope of any evaluation. By 'genetic epidemiology' we mean 'the study of genes and disease in populations, the design of epidemiological studies, and assessment of the impact of random variation, bias and confounding on their results. ${ }^{41}$ Essential components of an assessment include the burden of suffering from a potentially increased disease risk, epidemiological measures (such as the frequency of diseasecausing mutations in genes in different subgroups, and the contribution of genetic factors to the prevalence of disorders in populations), the accuracy of the test, and the comparison with alternative methods of detection.

Epidemiology deals first of all with measuring the effects of exposures (eg, family history of disease, lifestyle factors) upon disease risk. The primary goals are to obtain valid measures for the frequency of disease and exposures, as well as measures of association between the exposure and the causal effect.

In order to evaluate the assessment of tests and screening programs in quantitative terms, several measures have been developed and used by different authors (Box 3):

We will focus on one approach for the evaluation of outcomes of genetic testing that integrates quantitative measures: the ACCE model $^{92}$ (see also Burke and Zimmern ${ }^{93}$ ).

It is relevant to notice that the reviews performed under ACCE or EGAPP, as far as common disorders are concerned, focused on the monogenic subforms. If a test for low-risk alleles is to be assessed via the model, many issues in the model, as discussed below, currently, probably, cannot be properly addressed.

The array of studies leading to the development of the ACCE model dealt with the evaluation of test performance, quality control, and

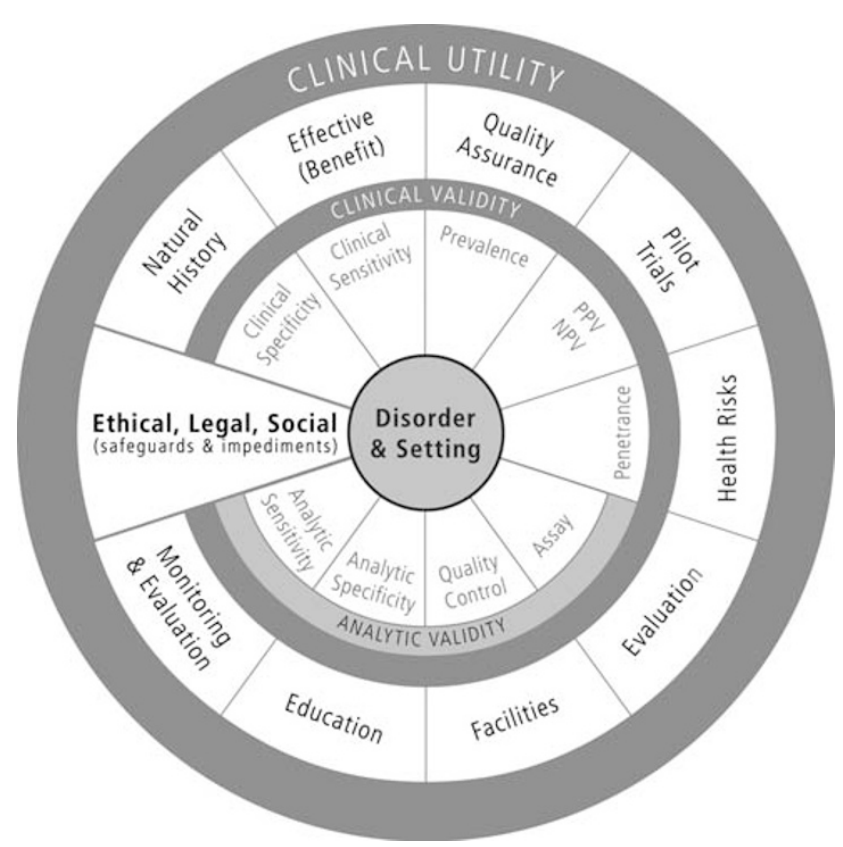

Figure 3 ACCE evaluation process for genetic testing. Source: Centers for Disease Control and Prevention (CDC). ${ }^{92}$

effectiveness. For that purpose, tests for several disorders (CF, hemochromatosis, VTE, breast and ovarian cancer, colorectal cancer) have been assessed regarding the components that later became the core elements of the ACCE model. It is a model process for the evaluation of information on emerging genetic tests, including the collection, evaluation, interpretation, and reporting of data about DNA and related testing services for genetically predisposed disorders. The main objective is to provide policy makers with up-to-date and reliable information for decision making.

The four eponymous components of the ACCE model (Analytic validity-Clinical validity-Clinical utility-Ethical, legal, and social implications) as well as their elements and relations to each other are displayed in the ACCE wheel (Figure 3). At the hub of the ACCE wheel are the clinical disorders and the setting in which testing is done. The evaluation process begins only after the clinical disorder and setting have been clearly established.

Specific questions 1-7 (see Appendix A) help to define the disorder, the setting, and the type of testing.

The CDC website ${ }^{92}$ explains the components analytic validity, clinical validity, clinical utility, and ethical, legal and social implications as follows:

'The analytic validity of a genetic test defines its ability to accurately and reliably measure the genotype of interest. This aspect of evaluation focuses on the laboratory component. The four specific elements of analytic validity include analytic sensitivity (or the analytic detection rate), analytic specificity, laboratory quality control, and assay robustness. Analytic sensitivity defines how effectively the test identifies specific mutations that are present in a sample. Analytic specificity defines how effectively the test correctly classifies samples that do not have specific mutations (although the term 'mutation' is used here, the terms 'polymorphism' or 'variant' may be more appropriate for certain situations). Quality control assesses the procedures for ensuring that results fall within specified limits. Robustness measures how resistant the assay is to changes in pre-analytic and analytic variables.' Specific questions 8-17 (see Appendix A) organize the information available to document analytic validity. 


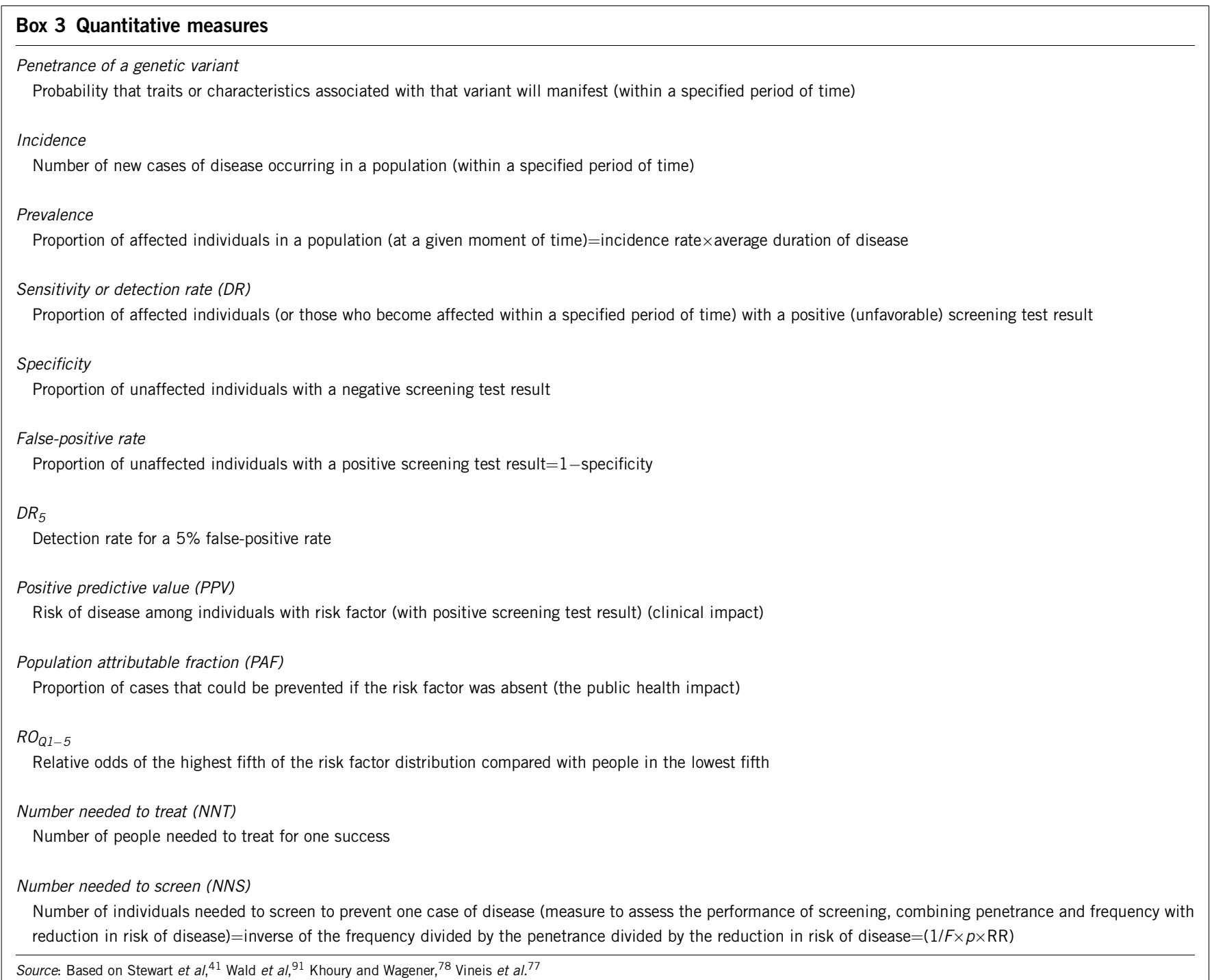

'The clinical validity of a genetic test defines its ability to detect or predict the associated disorder (phenotype). The four elements of analytic validity are all relevant to assessing clinical validity, along with six additional elements: clinical sensitivity (or the clinical detection rate), clinical specificity, prevalence of the specific disorder, positive and negative predictive values, penetrance, and modifiers (gene or environmental). Penetrance defines the relation between genotype and phenotype and allows the frequency of the clinical expression of a genotype (expressivity) to be determined. Clinical sensitivity measures the proportion of individuals who have a well-defined clinical disorder (or who will get the disorder in the future) and whose test values are positive. Clinical specificity measures the proportion of individuals who do not have the well-defined clinical disorder and whose test results are negative. Prevalence measures the proportion of individuals in the selected setting who have, or who will develop, the phenotype. The positive and negative predictive values more meaningfully define the genetic test performance by taking into account clinical sensitivity, clinical specificity and prevalence.' Specific questions 18-25 (see Appendix A) help organize the information available to document clinical validity.

'The clinical utility of a genetic test defines the elements that need to be considered when evaluating the risks and benefits associated with its introduction into routine practice. The natural history of the specific disorder needs to be understood so that such considerations as optimal age for testing might be taken into account. Another factor to be considered is the availability and effectiveness of interventions aimed at avoiding adverse clinical consequences (if no interventions are available, for example, testing may not be warranted). Quality assurance assesses procedures in place for controlling pre-analytic, analytic, and post-analytic factors that could influence the risks and benefits of testing. Pilot trials assess the performance of testing under real-world conditions. Health risks define adverse consequences of testing or interventions in individuals with either positive or negative test results. Economic evaluation helps define financial costs and benefits of testing. Facilities assess the capacity of existing resources to manage all aspects of the service. Education assesses the quality and availability of informational materials and expertise for all aspects of a screening service. Monitoring and evaluation assess a program's ability to maintain surveillance over its activities and make adjustments.' Specific questions 26-41 (see Appendix A) help organize the information available to document clinical utility.

'Ethical, legal, and social implications surrounding a genetic test are represented $\ldots$ by a penetrating pie slice, implying that the safeguards and impediments should be considered in the context of 
the other components.' Specific questions 42-44 (see Appendix A) help organize the information available to document ELSI issues.

\section{GENETIC TESTING AND SCREENING IN COMMON DISORDERS Forms of screening}

In many European countries, population screening is best known to the general public from the examples of breast cancer screening by mammography and PAP smears for cervical cancer screening. A defined segment of the population (women in a certain age group) is invited at a specified time interval.

Genetic screening programs exist in most European countries at least in the antenatal and neonatal phases of life. In this type of program, all pregnant women are invited for determination of their rhesus blood group, and assessment of risk of Down's syndrome (eg, by maternal serum screening) or neural tube defect (eg, by ultrasound screening). All newborns can participate in the programs using a blood test to diagnose treatable conditions as early as possible in order to avoid irreversible damage. Most newborn screening programs start with PKU. When adhering strictly to a special diet, affected infants will not develop severe mental retardation.

In some countries genetic screening programs exist that are aimed at high-risk subpopulations. Ethnicity or ancestry may be used to identify high-risk groups. In Finland for instance, indigenous Finnish newborns do not need screening for PKU because the disease risk in Finnish people is close to zero, while 'migrants' from other European countries have a higher risk. In southern European countries, such as Greece and Italy, screening programs for hemoglobinopathies, such as $\beta$-thalassemia, have been implemented decades ago. ${ }^{94}$

The genetic screening programs mentioned above either aim to provide people with information that enables them to make informed reproductive decisions (antenatal screening for Down's syndrome or neural tube defects) or relate to treatment or prevention options (anti$\mathrm{D}$ for rhesus-negative mothers who gave birth to a rhesus-positive child to prevent rhesus antagonism in the next pregnancy; diet for PKU for secondary prevention). These two different aims of screening programs need to be separated in discussions of societal implications.

Family structure is sometimes used to approach family members at high risk. This is referred to as cascade screening: first- or seconddegree family members of FH patients are systematically invited to have a test as well. Similar approaches exist for hereditary tumors.

In the age of genomics, screening in common disorders might be relevant later in life for screening for conditions where treatment or prevention is possible. People at high risk of developing a common disorder might benefit from personalized prevention programs to reduce their risk or postpone the onset of disease. Probably the number of applications that relate to informed reproductive decisions will be rather small.

\section{Testing and screening strategies}

One or several variants for one disease. Some genetic association studies report high relative risks for a specific genetic variant and conclude that 'genetic testing might become a future approach to identify individuals at risk. ${ }^{95}$ However, it remains unclear whether such genetic risk classifications have sufficient predictive value to be useful. ${ }^{69-73}$ Critical analyses of this kind of statement often reveal that the clinical validity of a test, focussing on one gene, let alone one allele only, is rather limited. ${ }^{4,96-98}$ Nevertheless, for diabetes, for instance, several susceptibility genes have been identified; yet, a singleton gene marker (TCF7L2 diabetes SNP essay) has been marketed for $\$ 500 .{ }^{4}$ Individuals who pay to have such a marker analyzed are only getting an 'ice-pick' genome view of susceptibility: other risk genes, modifier genes, protective alleles, as well as lifestyle factors are not being taken into account. At least the use of information on several genetic variants at a time (genetic profiling) would in most cases be more efficient, particularly if there is no gene-gene interaction. In this case, however, the distribution of log-odds ratios will be close to a normal distribution, with many people at close to average risk and very few at markedly higher or lower risk. As the variance of this distribution increases when more and more markers are included, an increasing number of people will have either a low or a high risk. ${ }^{98}$

One or several diseases. While genetic variants may indicate an increased risk for one disease, they may be associated with a decreased risk for others. Preventive and therapeutic interventions aimed at eliminating the negative effects of a single genetic variant for one disease may increase the risk of another disease. ${ }^{6,98}$

Some authors combine the state of knowledge regarding the genetics of several disorders and endorse a more global approach, for instance to cardiovascular risk. ${ }^{5}$

Historically, cardiovascular risk factors such as hypertension, BMI, age, smoking, fasting glucose level, salt intake, etc, have been used in primary care and public health (including occupational health) to tailor prevention programs to individual risk profiles. It has been claimed that public health professionals will increasingly have to incorporate genetic knowledge into their evaluation, at the population level, of the risk for CVDs. ${ }^{5}$ A list of specific recommendations to translate research findings into effective genetic screening programs would include the development of multidisciplinary collaborative research teams, the evaluation of how behavioral and environmental factors interact with genetic variants to influence CVD risk, to prepare proactively for effective genetic screening programs, and to educate researchers, clinicians, public health professionals and the general public. $^{5}$

Other authors do recognize the shift from understanding the biological processes to the complex biology of hemostasis, thrombosis, inflammation, endothelial dysfunction, and plaque instability. However, they propose to include only a limited number of novel biomarkers in preventive health care because data are scant. ${ }^{99}$

\section{Testing and screening for common disorders in Europe}

In the research for several disorders, associations between genetic variants and disease risk of potential clinical relevance have been established, for instance for hereditary breast and ovarian cancer, colon cancer, diabetes mellitus (MODY subtypes), thrombosis, cardiovascular disorders, celiac disease, and Alzheimer's disease. Although these examples again relate to monogenic subforms of common disease, they can nevertheless be used to reflect on what is possible or might be expected, and what might be perceived as major obstacles in using the new genetics in public health. Testing for these monogenic subtypes is being implemented in health care in some countries. A major criterion to decide what is or is not implemented in health care is clinical utility. For these examples, tests have a high or intermediate predictive value, and preventive interventions are available. It has to be borne in mind that screening for low-risk alleles in common disorders would raise some similar, but also some rather different issues because of the (much) lower predictive value, difficulties in establishing the utility of interventions, the setting of the test (commercial or health care), and ways of informing patients and the public (see also the section 'Genetic testing and screening in common disorders: what can we learn from $\mathrm{HH}$ and $\mathrm{FH}$ ?').

The presence of a germline $B R C A 1$ or $B R C A 2$ mutation may cause hereditary breast and ovarian cancer, which has consequences for 
treatment and prevention both in patients and in healthy family members. If a young woman is diagnosed with breast cancer, and is found to have a BRCA1/2 mutation, surgery might not be limited to removing the tumor alone. Prophylactic surgery (mastectomy, salpingo-oophorectomy) may be considered to reduce the risk of future breast and ovarian cancer. Family members may also wish to consider genetic testing. For healthy family members carrying the mutation, screening would start earlier, MRI can be offered, and prophylactic surgery can be considered.

Not only germline mutations may be of clinical relevance, but also gene expression may be relevant for health care, especially in cancer. In breast cancer treatment, response to Herceptin (trastuzumab) therapy can be determined using HER2 (ERBB2) testing of tumor tissue. ${ }^{100}$ In a more experimental setting, the expression of 70 genes appears to be a strong predictor of prognosis. ${ }^{101}$ The so-called Mammaprint ${ }^{\circledR}$ is a gene expression profiling test on tumors that helps discern who might profit from chemotherapy and who might not. ${ }^{102}$ BRCA1 and BRCA2 testing for inherited predisposition, as well as HER2 testing for therapy fit well within the existing practices of gynecologists and oncologists and have been implemented in regular health care. Clinical geneticists have mainly been involved in genetic counselling and developing guidelines for the high-risk groups of $B R C A 1$ and $B R C A 2$ mutation carriers.

Increasingly, molecular and immunohistochemical parameters are being included in the delineation of tumor subtypes in order to predict the response to therapy. Examples are determining microsatellite instability (MSI) and immunohistochemistry in colorectal cancer, which can indicate a reduced response to certain chemotherapeutic agents - but could also indicate an increased chance of Lynch's syndrome - and parameters of breast cancers such as basal phenotype, medullary histology, and 'triple-negative' receptor status (negativity for estrogen and progesterone receptor and the tumor marker HER-2/ neu), which could indicate an increased chance that the patient is a BRCA1 mutation carrier. Clearly, these tests are clinically useful in that they can alter patient management, but they may also indicate the presence of a strongly cancer-predisposing condition (Lynch's syndrome or BRCA1 mutation, respectively). Further evaluation with expression arrays will in the future delineate more subtypes of cancers, which may behave in different ways. These parameters may also reveal an increased chance that an individual may be a carrier of a germline mutation in a cancer susceptibility gene. Such tests therefore should only be done in connection with counselling regarding the possible implications and the need for further genetic tests should the test result be 'high risk'.

Regarding breast cancer, at present, much research is aimed at finding susceptibility genes of moderate and low penetrance in the wider population. Most of these polymorphisms alone have minimal effects upon risk, but when it becomes possible to test a combination of those genetic variants, this may result in the detection of profiles associated with a significant increase in risk of developing breast cancer. Only then could it be considered as a screening test for women to assess their relative risk. Currently, combining all the common genetic variants associated with breast cancer does not improve risk predictions compared with existing models using non-genetic risk factors. ${ }^{103}$ Pharoah et al ${ }^{104}$ argue that, currently, a test based on the known, common moderate-risk alleles does not provide sufficient discrimination to warrant individualized prevention (see also Ripperger $^{105}$ ). However, a screening test might in the future become relevant in the context of a population screening program. Thus, for instance, it could be used to identify higher-risk women who might be offered mammographic screening from an earlier age than the standard for population screening (from 48 or 50 years of age). Pharoah et al ${ }^{104}$ stress that, at present, many issues such as evidence, efficiency and acceptability of such a screening program still need to be established. For instance, MRI screening would be too costly unless targeted at patients at high risk.

The panel of susceptibility genes might differ between populations. ${ }^{105}$ For example, in Poland, a panel of markers has been established that might account for $92 \%$ of breast cancers in the Polish population, ${ }^{106}$ which could render screening in that particular population feasible.

Hereditary non-polyposis colon cancer (HNPCC) is known to be caused by germline mutations in several genes (MLH1, MSH2, MSH6, and PMS2). As with breast cancer, the presence of (highly penetrant) mutations may modify the therapeutic approaches taken both for patients and for their relatives. Family members can be tested for the specific mutation found in their affected relative. In an ACCE review it was mentioned that the Cancer Genetic Studies Consortium recommends monitoring of carriers of most notably the MLH1, MSH2, and MSH6 mutations using colonoscopy at 1-3-year intervals coupled with transvaginal ultrasound or endometrial aspiration annually for women, because women may develop endometrial and ovarian malignancies. ${ }^{107}$ When the mutation is absent, the risk of developing cancer is reduced to the population risk. The ACCE review suggests that because of the cost of DNA sequencing coupled with the low frequency of HNPCC mutations among individuals with colorectal cancer, testing all cases of newly diagnosed cases of colorectal cancer would not be indicated. Instead, a high-risk subgroup could be selected by using family history and/or MSI testing of the tumor. Drawbacks of this procedure would be a loss of cases, as only about $86 \%$ of individuals with HNPCC-associated cancer will have a positive family history, and about $90 \%$ of colorectal cancers caused by HNPCC mutations will display MSI. ${ }^{107}$

Similarly, in cardiac disorders, a distinction can be made between monogenic subforms, such as cardiomyopathies and primary arrhythmias, and multifactorial disease etiologies. ${ }^{108,5}$ Identifying risk carriers enables preventive and symptomatic treatment with medications as well as devices (implantable cardiac defibrillator (ICD), which may prevent sudden cardiac death (SCD) at a young age. Hypertrophic cardiomyopathy (HCM) is the most common autosomal dominant heart disease (with a prevalence of at least 1 in 500) and is the main cause of SCD while on the playing field. On the other hand, most HCM patients have a normal life expectancy. The causal mutation can currently be detected in approximately half of the index patients, facilitating cascade screening. In carriers, regular cardiac screening is possible, aimed at well-known risk factors for SCD (such as nonsustained ventricular tachycardias, massive hypertrophy, and a family history of SCD). The yearly risk of SCD in manifest HCM patients is $1 \%$, which might rise to $5 \%$ in those with two or more risk factors. Idiopathic dilated cardiomyopathy (DCM) is familial in about $30 \%$ of cases and is genetically very heterogeneous, making DNA testing difficult. Active cardiac screening and follow-up of first-degree relatives of DCM patients is advised, however, because early treatment is expected to improve the clinical course. Arrhythmogenic right ventricular cardiomyopathy (ARVC) is also known as a frequent cause of SCD during competitive sports, in males in particular. The clinical hallmarks of this disease are the replacement of the muscle of the right (and sometimes also left) ventricle by fat and connective tissue, with dilatation of the ventricle(s) and severe arrhythmias. The genetics of ARVC are very complicated, with several mutations detected in more than one ARVC gene in many patients, complicating cascade screening. Cardiologic screening (including MRI) is advised for all firstdegree relatives of ARVC patients. The only effective (preventive) 
therapy is an ICD. The long QT syndrome is the best-known example of a primary arrhythmia syndrome. The prevalence is 1 in 2000 . The molecular cause can be detected in up to $70 \%$ of the index patients. Treatment is mostly possible with beta-blockers in the two most prevalent forms of the long QT syndrome (caused by mutations in the KCNQ1 and $K C N H 2$ genes). On the other hand, in the long QT syndrome caused by mutations in the SCN5A gene (15\% of cases), beta-blockers may be harmful and ICD should be considered. Also, avoidance of certain drugs (ion-channel-blocking agents) that may prolong the QTc interval is useful to prevent arrhythmic events. Formerly healthy people using these substances sometimes get severely prolonged QT intervals and life-threatening arrhythmias, called the acquired long QT syndrome. In some of these patients, mutations of variants in the known long QT genes are detected.

In cardiogenetic outpatient clinics, cardiologists cooperate with (clinical) geneticists and genetic counsellors to systematically identify people at risk for SCD due to genetic factors. In the general population, family history of SCD at a young age in conjunction with phenotypic information could be used to detect patients at risk and establish whether the etiology is Mendelian or multifactorial. If a person under the age of 40 dies suddenly and unexpectedly and no cause can be detected, a genetic heart disease can be diagnosed in up to $40 \%$ of the families by cardiac screening of first-degree relatives. ${ }^{109}$ In presumed multifactorial disease, myocardial infarction with primary ventricular fibrillation for instance, a positive family history of SCD is found more often than in patients with myocardial infarction without VF, suggesting one or more underlying genetic factors. ${ }^{10}$ If possible, prevention can be targeted at the underlying risk factors. ${ }^{108,5}$

For diabetes mellitus, genetic testing may also modify or specify diagnosis and prognosis. MODY subtypes have an autosomal dominant pattern of inheritance. Recurrence risk is thus higher than in other types of diabetes. Furthermore, MODY subtypes tend to start at an early age but may have different clinical implications. MODY2 due to glucokinase mutations is often mild. The prevalence of microvascular complications is lower than in late-onset type 2 diabetes. The hyperglycemia is usually responsive to diet. MODY3 due to HNF1A mutations is a more severe form of diabetes. These patients are more sensitive to the hypoglycemic effect of (oral) sulfonylureas. Thus, insulin treatment may be postponed. MODY subtypes 2 and 3 together account for the majority of MODY cases. The rare subtype MODY5 due to $H N F-1 \beta$ mutations is associated with a rapid reduction in $\beta$-cell mass and requires rapid insulin treatment. This highlights the importance of recognizing subtypes to optimize their clinical approach. ${ }^{111}$

For type 2 diabetes mellitus, in 2009, SNPs in 24 different genetic loci had been identified. ${ }^{112}$ Most of these genetic loci are found to be associated with the insulin secretion pathway rather than insulin resistance. However, these loci do not seem to offer greater predictive value in determining diabetes risk than do commonly used phenotypic risk factors and family history. ${ }^{112}$

For celiac disease, the relevance of genetic testing (HLA-DQ2 and DQ8 subtypes) for prevention is debated in the broader context of the pros and cons of early diagnosis through screening. ${ }^{113}$ Celiac disease is an important health problem for the individual and the community because of its high prevalence, associated specific and non-specific morbidity, and long-term complications, of which the most important are gut malignancy and osteoporosis. ${ }^{113}$ The vast majority of cases remain undiagnosed. The prophylactic benefits of early diagnosis through screening for individuals with mild enteropathy may be low, and there is no consensus on whether or not to treat individuals with mild symptoms.
Alzheimer's disease is known to be associated with variation in the apolipoprotein $\mathrm{E}$ gene $(A P O E)$. The therapeutic response in $\mathrm{AD}$ is genotype-specific, with $A P O E^{\star} 4 / 4$ carriers representing the worst responders to conventional treatments, ${ }^{114}$ such as donepezil, rivastigmine, galantamine, and memantine. This treatment aims to slow down the disease process ${ }^{115}$ and is administered in some countries, most notably the United States. Furthermore, the association of the APOE-4 allele with specific genetic variants of other genes (eg, CYP2D6, angiotensin-converting enzyme (ACE)) negatively modulates the therapeutic response to multifactorial treatments affecting cognition, mood, and behavior. ${ }^{114}$ Pharmacogenomic testing could thus help to individualize treatment in patients already showing symptoms of AD. This application should be distinguished from susceptibility testing for $\mathrm{AD}$ in the general population. The risk of developing $\mathrm{AD}$ for $\mathrm{APOE}^{\star} 4 / 4$ carriers is higher compared with noncarriers; yet, more than half of the carriers will not develop AD. As there are no effective ways to prevent or cure Alzheimer's disease, population screening is not desirable.

In behavioral and neuropsychiatric disorders, associations between gene variation and behavior have been shown to be extremely difficult to establish. Especially in this area, social and ethical issues regarding stigma and identity are further major obstacles for genetic testing.

Venous thrombosis is caused by a combination of genetic and environmental factors. Although different mutations influence coagulation, FVL is the most common genetic risk factor for venous thrombosis. About $5 \%$ of the white population carries this mutation. Currently, the test for this mutation is one of the world's most heavily used genetic tests.

It has been debated whether testing for FVL might be relevant in specific situations such as before surgery, during pregnancy and childbirth, and before prescribing oral contraceptives. ${ }^{116}$ When a patient already has an established venous thrombosis, testing might be relevant, as recurrence risk is higher in carriers. However, the risk of developing venous thrombosis is still low and should be weighed against the consequences for the use of contraceptives and unwanted pregnancies. Cohn et al ${ }^{117}$ suggest that testing should be performed with restraint because there are no benefits and no direct consequences in terms of treatment. A US Department of Health \& Human Services technology assessment on testing for FVL and prothrombin G20210A concluded: ${ }^{118}$ "There is no direct evidence that testing for these mutations leads to improved clinical outcomes in adults with a history of VTE or their adult family members. The literature supports the conclusion that while these assays have high analytic validity, the test results have variable clinical validity for predicting VTE in these populations and have only weak clinical utility' (see also Segal et al ${ }^{119}$ ).

These examples of the potential use of predictive and susceptibility testing and screening for common disease show the relevance for three domains: diagnosis and prognosis, individualized treatment and disease management, and disease prevention. For these three domains, genetic information could be used in current practices, or in other cases, new organizations or services might need to be established. Besides, for all the three domains, the role of clinical geneticists in educating other health-care professionals or in helping reorganize health care merits discussion. As the examples show, it can be necessary to select a high-risk group in which further testing or monitoring is indicated, as in some arrythmias. In some other instances, the role of geneticists may be to discuss the limited clinical relevance and thus avoid implementation in health care. However, the criteria for selecting and the cases lost by selecting may pose still further problems that need to be addressed when contemplating balancing the pros and cons of introducing genetic testing or screening 
in a particular health-care setting. Furthermore, it should be noted that, for most common disorders for which genetic factors are being identified, susceptibility testing for low-risk alleles, let alone susceptibility screening, is currently not indicated for reasons of clinical validity and utility, as explained in the section 'Gene-disease associations'.

\section{Genetic testing and screening in common disorders: what can we learn from $\mathrm{HH}$ and $\mathrm{FH}$ ?}

$\mathrm{FH}$ is considered to be a monogenic condition, but there might be relevant lessons for screening in common disorders. $\mathrm{FH}$ is a highly penetrant autosomal dominant disorder caused by a mutation in the low-density lipoprotein receptor gene. Prevalence is estimated to be 1 in 500 in Western countries. Cardiovascular problems can be already present in early adulthood, and preventive medication (statins) can be offered to reduce the risk. In several countries, family or cascade screening has been introduced for FH. ${ }^{120}$ There may be an analogy with some common chronic disorders, such as diabetes, where it is becoming increasingly clear that having a family member with a certain disorder can be an important risk factor in developing a disorder. ${ }^{121}$ Thus, forms of cascade screening or collecting data on family history in FH might be explored to see what aspects might be relevant for genetic screening in common disorders.

In the Netherlands, since 1994 a genetic screening program for $\mathrm{FH}$ has been organized as a cascade screening, which has been upscaled to the world's first national genetic screening program for FH in 2004. ${ }^{122}$ The contacting and testing of family members is organized via an intermediary organization that could act as an example for organizing cascade screening: the Foundation for Tracing Familial Hypercholesterolaemia $(\mathrm{StOEH})$ that functions as a registry. After an index patient has been found and diagnosed, StOEH organizes the contacts with the family members and collects blood samples for testing. This solves several issues of time management, central data gathering, quality control, and informing patients and relatives. Genetic field workers have an important role in informing patients and family and making family screening possible.

The relation with primary care is a key issue: StOEH can perform effectively only when a sufficient number of index patients are found.

Whereas also hereditary hemochromatosis $(\mathrm{HH})$ can be considered to be a monogenic disorder, because of the low-to-moderate penetrance of the associated variant, screening using the genetic test would resemble screening for risk status as in screening for multifactorial or common complex disorders, such as diabetes. Therefore, experiences with and discussions about $\mathrm{HH}$ screening could provide insights when reflecting on screening for those common disorders for which new tests might be developed in the future.

The mutations underlying $\mathrm{HH}$ are among the most common autosomal recessive mutations in people of Northern European origin. Hemochromatosis is characterized by iron overload in tissues and organs of male patients usually over 40 years, and in female patients after menopause. Currently many patients are detected only after severe organ or tissue damage has occurred.

Several tests are available for measuring iron levels in serum. ${ }^{123} \mathrm{~A}$ DNA-based genetic test became possible when in 1996 the C282Y and H63D mutations were discovered in the HFE gene. In people from Northern European origin, about $85 \%$ of people clinically diagnosed with hemochromatosis are homozygous for the C282Y mutation. About 1 in 200 of this population is homozygous for the $\mathrm{C} 282 \mathrm{Y}$ mutation. ${ }^{124}$ In Italy, homozygosity occurs less frequently than in Portugal and Northern European countries, whereas in Northern Ireland homozygosity is estimated to be around 1 in $100 .{ }^{125}$
Arguments in favor of implementing population genetic screening for $\mathrm{HH}$ are the fact that a diagnostic test and a treatment are available and that timely intervention (phlebotomy or blood-letting to remove excess iron) can prevent morbidity. Yet, at the 1997 US Centers of Disease Control and the National Human Genome Research Institute Consensus Meeting ${ }^{125,126}$ it was stated that screening was not recommended, as there were several issues that needed to be resolved first. Knowledge of the penetrance of the disorder was lacking, as well as evidence on the optimal time of screening, treatment, and psychological consequences of screening. Not all homozygotes develop iron overload, and not all patients with iron overload develop organ and tissue damage. ${ }^{127}$ Debate on the clinical expression is still going on. In 2002 Beutler et al ${ }^{128}$ argued that penetrance is actually quite low and they estimated that less than $1 \%$ of homozygotes would develop fullblown clinical hemochromatosis. This would result in less favorable calculations for cost effectiveness. However, in 2008, Allen et al ${ }^{129}$ reported, for Australia, iron-overload-related disease in more than $28 \%$ of male $\mathrm{C} 282 \mathrm{Y}$ homozygotes. This confirms earlier findings from a German pilot study aimed at insurants of a German sickness fund, in which 8 out of 34 newly diagnosed homozygous C282Y mutation carriers were found to show iron accumulation with further signs or symptoms of HH. ${ }^{130,131}$

Screening of first-degree family members, most notably siblings, of affected individuals has been suggested and has been implemented in many countries. Siblings have a $25 \%$ chance of having the same predisposing genotype and are expected to have a higher risk of morbidity in case of a seriously affected family member, ${ }^{132-135}$ although the extent of risk for the family members of affected patients is not yet clear. ${ }^{136-138}$ At the same time, raising awareness among health practitioners about detecting early symptoms of $\mathrm{HH}$ has been promoted. ${ }^{123,139,140}$ Studies from the United Kingdom and the Netherlands show, however, that often patients showing symptoms of $\mathrm{HH}$ or family members of $\mathrm{HH}$ patients are not yet being offered a test, despite protocols that recommend testing. ${ }^{134,141}$ Therefore, in families where the mutation has an impact, hemochromatosis is still underdiagnosed. Possibly medical specialists are not used or inclined to deal with the familial aspects of the disorder, or are less prone to focus on prevention. In that case, closer contacts with clinical geneticists might prove helpful in educating health-care professionals and organizing better screening and case detection practices.

Powell et $a l^{135}$ state that the most cost-effective methods of early case detection are family (cascade) screening and evaluation of potential cases by primary-care physicians. Pietrangelo ${ }^{142}$ also points to the role of primary care and the importance of screening patients for iron overload. He estimates that it will be possible to extend this screening to all patients seen in primary care: 'The rationale is similar to that for routine cholesterol screening and the prescription of dietary restrictions and drug therapy when risk is verified in the absence of signs of CVD. When the results of iron-metabolism tests are abnormal, clinical biochemical values should be monitored; if genetic tests are positive for $\mathrm{HH}$, prophylactic iron depletion should be considered.' Njajou et $a l^{143}$ also propose a two-phase population screening program, based on establishing serum iron levels biochemically and then a genetic test for people with elevated serum iron parameters.

Genotype-based screening has also been reported to be cost effective. For example, the above-mentioned German pilot study was aimed at insurants of a sickness fund who were tested for the C282Y mutation. ${ }^{130}$ Recently, a systematic review concerning genotyping for the $\mathrm{C} 282 \mathrm{Y}$ mutation underscored that DNA testing for $\mathrm{HH}$ in at-risk populations has clinical validity and may have clinical utility; in addition, two studies suggesting cost effectiveness were found. 
However, the review highlights the limitations of the literature and the methodological difficulties associated with evaluating this genetic test. ${ }^{144}$ For Germany, a recent review discusses the cost effectiveness of different strategies of detecting patients via cascade screening, population screening, and genotyping. ${ }^{145}$ It confirms the cost effectiveness of the current German policy to screen only for at-risk individuals, but suggests to conduct a DNA test after elevated iron levels have been found, instead of performing two transferrin saturation tests. ${ }^{145}$

Finding, counselling and testing sibs is a time-consuming and specialized activity. If the numbers of people applying for testing increased (as is expected to be the case for $\mathrm{HH}$ and for other disorders for which an important genetic component will be established), current facilities for testing and counselling may not be adequate, either in primary care or in specialized medical care. More systematic approaches might be organized in diverse ways, depending on the health-care organization and system and practices of referral in a specific country.

In the Netherlands, it has been suggested that the model used by the Foundation for Tracing Hereditary Hypercholesterolaemia (StOEH) might be appropriate for $\mathrm{HH}$ as well. ${ }^{142}$ In considering cascade screening as organized by StOEH as an exemplar for susceptibility screening, it should be noted that screening for FH differs from both susceptibility screening and screening for $\mathrm{HH}$. The fact that $\mathrm{FH}$ is a highly penetrant autosomal dominant disorder favors the effectiveness of cascade screening.

In tackling the question of how to improve contacts between primary and specialized care, and how to optimize the system of referral of patients, it is not enough to address the knowledge gap between family doctors and specialized care. Indeed, knowledge about genetics is lacking. ${ }^{147}$ However, better knowledge might not be sufficient for referral and follow-up. Primary care may have its own practical considerations and interests that need to be understood and considered. ${ }^{32,148}$ In making better use of genetic knowledge for public health purposes, not only knowledge transfer but also practice needs to be taken into account. Intermediary organizations such as $\mathrm{StOEH}$, as well as intermediary professionals, such as genetic nurses and genetic field workers, and, last but not least, professionals active in the clinical genetic setting, such as genetic counsellors, might be helpful in bridging the gap between primary care and specialized care.

\section{THE ECONOMIC EVALUATION OF GENETIC TESTS}

Genetic testing for screening purposes as part of the health-care system typically aims at preventing or reducing deterioration in health. Economic evaluation of genetic tests addresses very heterogeneous aspects: the uncertain characterization of genes involved in the etiology of complex diseases, the partial knowledge about which people are at increased risk and about the likelihood that an inherited predisposition may result in the development of disease, as well as the incomplete potential to predict the effectiveness and cost of an intervention.

As previously mentioned, the evaluation of a health technology such as genetic screening for health purposes can be conducted by instruments specifically directed at the technology of screening, like the WHO criteria established by Wilson and Jungner, ${ }^{22}$ or at the health-care setting and the genetic disease, like the $\mathrm{ACCE}^{11}$ or the EGAPP $^{149}$ framework. Specific instruments are usually highly sensitive to the character of the technology under investigation. The clinical utility domain of the ACCE framework explicitly includes economic evaluation. Alternatively, more generic health economic methods ${ }^{150-152}$ that have been applied to a broad range of health technologies can and have also been applied to evaluate genetic tests. ${ }^{153}$ Health economic evaluations explicitly take into account the consumption and savings of scarce resources implied by the application of a health technology. In addition to the question of whether or not a technology can be considered effective, they provide guidance on whether the investment in this technology provides sufficient value for money as compared with alternative available uses of a given budget.

The present chapter is dedicated to the assessment of genetic testing by health economic evaluations and the appropriateness of genetic tests for screening programs that might also be derived from those evaluations. The major tasks of economic evaluations are to identify, measure, value, and compare the inputs (costs) and outputs (effects) of the alternatives being considered. ${ }^{154}$ Given the limited healthcare resources in general and in particular the consequent inability to produce all desired outcomes - or even implement all efficacious therapies - choices must be made concerning the implementation of genetic testing services. Thus, economic evaluations of genetic tests can help to ease the decision-making process by providing information about both costs and consequences of alternative courses of action, as well as their appropriateness and utility in the context of genetic screening programs.

Effectiveness and cost effectiveness of genetic testing and screening Health economic evaluation is increasingly used to inform policy makers about prioritizing calls on a constrained health-care budget. The requirements for full economic evaluations include the need to consider all appropriate evidence, to compare new technologies with all relevant alternatives (in terms of costs and consequences), and to reflect uncertainties within the analysis. ${ }^{152}$ Early identification of a disease predisposition conferred by a genetic polymorphism might help to lessen suffering and premature death while reducing the costs of medical treatments and therapies. ${ }^{155}$ Systematic screening programs are more likely to be cost effective if a disease is relatively common or the risk for disease sufficiently increased. At the same time, genetic tests (that reliably provide information about an individual's susceptibility to disease) as well as effective treatments for the predisposed disease have to be available.

Effects. The starting point of health economic evaluations is to assess a technology's benefit and to determine whether the benefit outweighs its potential health-related harms. A general difficulty in identifying and assessing an initial genetic test originates in the fact that test results in the first instance represent no primary clinical effects but intermediate outcomes. This is caused by the fact that they can only help in determining an appropriate treatment or prevention leading to final health outcomes. Evaluations as part of the assessment of the clinical utility usually deal with final health outcomes that can be measured either in natural units (eg, life-years gained (LYG)) or in preference-based measures (eg, quality-adjusted life-years (QALY)). Genetic susceptibility screening in common disorders aims at offering opportunities for early diagnosis, preventive (primary or secondary) strategies, or informed choice. The result of a single genetic test may allow for a more personalized treatment being applied in case of a (true) positive test result. At the same time, it may produce changes in the tested individual's behavior, bringing about a totally different lifestyle. Also, the impact of knowledge about genetic status on a patient's quality of life in general and on his or her emotional, physical, and social situation in particular has to be considered. ${ }^{156}$

The effectiveness of a genetic test is determined by the prognostic significance of the test result. This has to be distinguished from the efficacy, which is described as the diagnostic ability of a genetic test, 
that is, the ability to accurately detect the genetic variation it was designed to identify. For the economic evaluation of genetic tests, it is the effectiveness that has to be factored into the analysis, which besides the availability and effectiveness of prevention - depends on the degree of association between a genetic variation and the physical manifestation (phenotypic characteristic). The penetrance of a genetic variant itself has an impact on both the clinical and economic outcomes. ${ }^{157}$ The same goes for the sensitivity and specificity of a genetic test: The more sensitive and specific a test is, the more effective it is regarding potential clinical benefits. An advantage of (full) economic evaluation is the consideration of characteristics beyond the mere sensitivity and specificity of a genetic test. The general aim is to measure all effects on health to determine all benefits of a test's implementation even if the intermediate effect (ie, the test result) has no impact on patient-relevant effects (eg, LYG).

Another unknown factor is the potentially harmful behavioral change caused by the impact of knowledge of genetic status on the tested individual's behavior: ${ }^{157}$ Patients with identified genetic variants might exhibit a change in behavior, while patients with no major genetic variants identified might adopt a more careless attitude with regard to their lifestyle and the exposures potentially related to disease risk. Regarding common disorders with a considerable impact of environmental factors on the disease onset, this might prove counterproductive if exposures have a higher effect on the development of disease than the genetic predisposition. If people who have been tested within the scope of a genetic screening program obtain a negative test result, the development of an adverse behavior would again increase the burden on the health-care system because a shift of risk occurred from the subgroup of genotypic high-risk individuals to environmental high-risk individuals.

To facilitate a meaningful health economic evaluation of genetic tests, the effects of testing have to be established in terms of end points that are relevant to patients and decision makers. Different approaches have been proposed to capture such benefits. According to the ACCE framework, the clinical benefits can be evaluated within the scope of the analytic validity and clinical validity, which address a broad range of aspects, including cost effectiveness and the impact on quality of life. However, it has been claimed that considerations regarding the clinical utility should not only refer to clinical end points but also include psychological, ethical, legal, and social issues, because 'they contribute to the net balance between benefits and harms of genetic testing for tested individuals, their families, and the population at large, ${ }^{158}$ so that the term 'clinical utility' may be too narrow to capture the range of decision-relevant benefits and harms. The patientrelevant end points for evaluating the usefulness of a genetic test can also be determined by the ability to reduce the population burden of morbidity, mortality, and disability. ${ }^{159}$ Khoury et al $l^{79}$ defined the benefits from a reduction of disease burden in the population (based on genomic profiling) as public health utility. Thereby, the population impact is expressed, implying that the reduction in disease burden can be measured after removing the exposure responsible for the disease burden. However, a test applied to identify gene mutations in a large proportion of the population is not necessarily useful. ${ }^{35}$

To quantify the magnitude of improvements in health outcomes in a way that makes it comparable to the magnitude of costs in a costeffectiveness analysis, it is necessary to apply a cardinal and, if health gains are compared across conditions, a generic measure (eg, QALY, LYG, etc) to combine the impact of disease, disability, and premature death. Besides the potential effects of genetic testing on the quality of life - due to early identification of disease-associated genetic variants and taking appropriate measures to reduce morbidity - there may also be effects on life expectancy. These effects depend on the individual's age at the time of the test (or potentially the time of the diagnosis), the treatment options, and the therapeutic outcomes. The onset of symptoms can be delayed or even avoided and the quality of life (at this point, ie, the overall assessment of the excellence of a life) observably improved. As the long-term prognosis for patients with genetic disorders improves over the coming years as a result of expected advances in treatment, so also will the effectiveness of genetic testing services increase.

The effects of genetic testing are subject to uncertainty. Given that, typically, no single study is available that establishes the long-term cost effectiveness of a screening program as a whole; modelling has to be utilized to provide a structured quantitative synthesis of the evidence of different aspects of screening (eg, test sensitivity, health effect of prevention, adherence to prevention), including their joint parameter uncertainty.

For tests that have no tangible health effects but may nevertheless be of positive outcome to patients and consumers, benefit may also be captured in terms of the individual's willingness to pay (WTP). It has been proposed that WTP may be a better outcome measure than attempts to establish clinical effects. ${ }^{160}$ However, in the face of scarcity, there are competing uses of health-care resources across a wide range of conditions, including, for example, new treatments for severely ill cancer patients. In such a competitive situation, it is unclear whether health-care payers decide in favor of funding for a large number of upcoming genetic and genomic tests with unproven clinical benefit. Therefore, clinical utility is likely to remain of high importance in the evaluation of genetic tests.

Costs. The costs that have to be considered in the economic evaluation of genetic testing and screening can be split up into medical resources consumed by the health-care intervention (direct medical costs or other non-medical resources consumed by the intervention (direct non-medical costs, eg, travel cost)) and productivity losses (if an individual is absent from work and is therefore not able to contribute his or her usual productivity).

Direct medical costs include costs for test kits used to obtain primary results and to eventually confirm those results. Because economic evaluations always have to compare alternative options, not only the costs of a genetic test but also the differences between the testing alternatives and the differences in treatment costs (depending on individuals being tested or not) have to be considered. Furthermore, staff costs need to be considered, which refer to the workload caused by a genetic test, including the costs of genetic counselling, if needed.

Whereas the costs for test kits as well as costs for laboratory staff and genetic counsellor costs can be rather easily assessed, the treatment and follow-up expenditures are of greater complexity; they might exceed the direct costs for purchasing the test notably. This may include a range of unnecessary follow-up testing in case of positive test results, even if tests are directly provided to consumers at no initial cost to the health-care payer. ${ }^{161}$ Frequently, the efforts required for additional medical care and follow-up are not known at the time of the test, so that only rough estimates of future costs are possible. ${ }^{162}$ Additionally, problems may occur when introducing new screening programs into clinical practice. If adequate requirements at system level are not fulfilled and new structures have to be introduced (to identify the appropriate target group, implement screening, provide counselling or support if needed), additional costs might result, as well as a decrease in the potential health impact, which should be considered in a comprehensive evaluation of genetic screening programs. 
Cost effectiveness. The decision about whether or not to perform a genetic test emanates from consideration of the consequences, including the benefits (outputs) and costs (inputs) of alternative medical interventions. The results of economic evaluations can only be interpreted by reference to an external standard (such as the result from other, independent programs, the cost-effectiveness threshold reflecting the value for money of the existing programs).

Four types of economic evaluations can be distinguished:

(1) Cost-minimization analysis (CMA) compares only the costs of treatment alternatives. CMA is used in situations where two interventions are known to incur equal health outcomes. This could be the case, for example, for different laboratory procedures delivering genetic test results with equal sensitivity and specificity. However, typically treatment alternatives differ not only in terms of costs but also in terms of effects.

(2) Cost-effectiveness analysis (CEA) establishes the cost per health outcome for the treatment alternatives measured in natural units (eg, cases detected or LYG). This can be used, for example, in the comparison of different screening strategies for a hereditary disorder like hemochromatosis. ${ }^{145}$ CEA is the most frequently used form.

(3) Cost-utility analysis (CUA) assesses the cost per outcome measured in utility values (eg, QALYs). Besides a comparison of different screening strategies within one medical condition, they can additionally be used to compare the cost effectiveness for very different screening programs or other medical interventions. This can then be used to establish whether the allocation of funds for this test provides a higher benefit than alternative uses of the scarce resources.

(4) Cost-benefit analyses measures the effects of tests in terms of how much individuals would be willing to pay (WTP) for them. This allows including non-health benefits into the analysis. Also, it allows for a comparison of value for money beyond the healthcare sector.

In a very broad review of economic evaluations of genetic tests, ${ }^{163}$ more than half of the 62 economic evaluations were CEA. The remainder were CUA as well as analyses based on a comparison of testing costs with costs potentially avoided, particularly owing to terminated pregnancies after diagnosis of severe hereditary disease. These analyses are frequently termed cost-benefit analyses; given that they are restricted to a comparison of costs rather than assessing benefit in terms of WTP, CMA may be an appropriate term.

To assess the methodological quality of economic evaluations, guidelines have been developed, for example, a checklist by Philips et $a l^{164}$ for evaluations based on decision analytical models or the CHEC list by Evers et al ${ }^{165}$ for trial-based evaluations (see also Appendix B).

Given time and resource constraints, it seems hardly possible to evaluate most of the genetic susceptibility tests extensively. A key question - regarding genetic testing in common disorders under public health auspices - that has to be answered is whether the clinical validity of testing for common disorders will be sufficient to warrant the use of limited public funds for both testing in healthy individuals and further research (eg, large-scale genome-wide association studies). ${ }^{166}$

Given that an increasing number of genetic tests are easily available, for example, via the internet for private use (ie, 'direct-to-consumer genetic testing' (DTC)), a second key question is to evaluate the potential harms of tests for testing or screening purposes. The more the tests are available due to a direct-to-consumer advertising, the more likely it is that market mechanisms (ie, competition, individuals' WTP) will regulate the uptake of genetic tests, which may also lead to problems of uncertainty and information asymmetry, which are well known to distort markets in the health-care sector. ${ }^{167}$ Especially in the case of common disorders and the need for identifying more than one gene variant or disease-causing mutation, a simple risk prediction is very likely not to be sufficient. This is caused by the fact that counselling might be indicated in some cases and/or that genetic testing in common disorders with low penetrance cannot completely predict the risk of disease (onset). Here, evaluations of genetic tests to identify (potential) effective and efficient genetic tests as well as evaluations of potential harms may be necessary to help policy makers develop market regulations that protect individuals' health and the social welfare.

\section{The health economic evidence base for genetic testing for common disorders}

This section is about identifying the health economic evidence on DNA-based testing and screening in common disorders, including the 'monogenic subset' of diseases within this group. Genetic tests that were considered are those that involve DNA-based genetic testing (aimed at detecting specific variants in the DNA sequence). ${ }^{41}$

The area under investigation was a number of recently published reviews covering economic evaluations of genetic testing. ${ }^{155,163,164,168-170}$ An additional conference poster presentation updated the economic evaluations of pharmacogenetic tests. ${ }^{171}$ Given the intentions of a NoE to consolidate existing research rather than to conduct original studies, this review draws upon the studies quoted above. All evaluations mentioned were extracted (evaluations of prenatal testing were excluded), and we assessed whether the studies met the definitions given in the previous chapters. The types of economic evaluation most commonly used in this context are costeffectiveness analysis (results represented in costs per LYG) and costutility analysis (results represented in costs per QALY). The main factors determining the cost effectiveness of genetic testing in common disorders were the prevalence, mortality risk, and the test costs.

The health economic evidence on genetic screening is limited because only few conditions have been properly evaluated. None of the identified reviews explicitly targeted genetic polymorphisms. To account for potentially omitted or recently published studies, a complementary search targeted the intersection of the concepts 'health economic evaluations' and 'polymorphisms' in several databases (PubMed and Cochrane, including the Centre for Reviews and Dissemination databases DARE, NHS EED, HTA). Given the high quality of PubMed indexing ${ }^{153,172}$ and large difficulties in representing an unknown number of conditions and mutations in a systematic search, this query was conducted by Medical Subject Headings. Details of the search are given in Appendix C1. All studies of common disorders identified by extraction of reviews or the supplementary search are given in Appendix C2.

The query revealed that hardly any health economic evidence exists that supports the clinical utility of DNA-based testing and screening in common disorders with low penetrance (see Appendix C2). Most research done so far considers rare diseases or the highly penetrant 'monogenic subset' of diseases within this group (eg, breast cancer).

The findings generally confirmed the results of a study by Rogowski and Langer ${ }^{155}$ dealing with genetic tests and their application in screening programs, which revealed that only screening for rare diseases - familial adenomatous polyposis (FAP) and retinoblastoma - has been calculated to be cost saving. Regarding FAP, the financial 
benefits mainly arose because close relatives who are negative for a causative mutation identified in the index case were excluded from increased colonoscopy and sigmoidoscopy on the basis of a positive family history. The effectiveness of genetic testing for retinoblastoma in relatives was shown by a comparison with conventional screening using ophthalmological examinations under a general anesthetic. Testing a family member in this case is comparable to a cascadescreening approach. In addition, the cost effectiveness of systematic screening for genetic contributors to hereditary non-polyposis colorectal cancer is likely to fall below the typically cited benchmark values, provided that certain criteria for diagnosis are applied (eg, Amsterdam criteria, Bethesda criteria). After immunohistochemistry testing, testing for HNPCC mutations may also have favorable costs per LYG in all patients with colorectal cancer. ${ }^{173}$

Evidence for the benefit of a population-wide screening for hereditary diseases, for example, hereditary breast and ovarian cancer, was generally weak. If at all, screening may only meet the frequent thresholds of cost effectiveness in case of specific populations in which a founder effect is present, for example, in the case of BRCA screening in Poland. ${ }^{174}$ Also, many of the studies may be outdated because they do not account for the currently much lower test costs than years ago, but equally not for the most recent evidence of effectiveness. The evidence on screening for FH is partly contradictory; the comparable studies proved the effectiveness of phenotypic screening.

The values per LYG or QALY could diverge widely. Partly, this may be because of weak epidemiological evidence. Before the evaluations can be used in decision making, the results have to be examined more closely to determine whether they are due to differences in the study design or due to different health-care settings that might influence the cost per LYG. Especially against the background of the historical development of different health-care systems, differences in the evaluation of health-care benefits and in thresholds for the implementation of new interventions seem evident. ${ }^{175}$

But as it is complex - if not impossible - to fully evaluate all or even most of the genetic susceptibility tests that are available, given the limited funds for health care and health research, also applied research for novel genetic tests needs prioritization. ${ }^{173}$ Criteria for selecting tests may include the penetrance and prevalence of the specific disorder, as well as the nature of the test, its purpose, and the population in which it is to be carried out. This may imply that tests for common disorders (with higher prevalences) are more likely to be assessed than tests for rare disorders, as the costs for a full evaluation are better justifiable because of the higher number of individuals involved, ${ }^{93}$ at least if there is some prior probability of the tests being cost effective. Given the results of recent genome research and the search for major risk factors in common disorders, we noted that only a small proportion of the total genetic risk can be accounted for by the risk factors identified to date. ${ }^{176}$ The genetic heterogeneity of multifactorial disorders poses the challenge of distinguishing disease-causing variants from functionally neutral ones. The association between a genetic variant and the respective disorder has to be sufficiently established by genetic epidemiological studies: as long as no evidence exists on the effectiveness of genetic testing in common disorders, the evaluation of cost effectiveness is not likely to produce meaningful outcomes. And yet, after its initial introduction onto the market or into clinical practice, the performance of a genetic test with sufficient priority for further research should be assessed over time. Ideally, this has to include a pre-market review (to ensure a test's potential to achieve its purpose) as well as a post-market assessment (to guarantee adjustments if needed).
The identification of new risk factors has not yet revealed many novel insights into the pathogenesis of common diseases, as associated markers were usually found in non-coding regions or in genes with unknown function. Furthermore, the limited resolution of association and linkage analysis so far has led to the fact that responsible sequence variants could not be precisely mapped. ${ }^{176}$ If this challenge of precisely mapping disease-causing variants was met, the evaluation of appropriate genetic testing would be associated with a lower level of complexity and uncertainty and the effectiveness of genetic tests could be assessed more easily - as can be observed in Mendelian disorders up to now. The health economic evidence on DNA-based testing and screening in common disorders focuses on the Mendelian subsets of common diseases. It is particularly noticeable that even with full HTA reports not all aspects (among others, clinical, social, ethical, economic aspects) are considered when evaluating genetic tests. Especially the lack of social and ethical considerations stands out, given the special importance of these issues to that part of the population being offered genetic screening or testing. Therefore, it is crucial to take a closer look at social and ethical aspects in addition to medical and economic considerations.

However, only a small proportion of individuals will possess the constellation of genotypes needed to increase the positive predictive value significantly. Furthermore, the complexity of genetic and environmental interactions and the interdependence of varying risk factors might never be represented in association studies. ${ }^{177}$ Thus, to screen entire populations for genetic susceptibility to common diseases under public health auspices would be very expensive and potentially even harmful.

\section{GENETIC TESTING AND SCREENING FOR COMMON DISORDERS IN HEALTH CARE: ETHICAL, LEGAL, AND SOCIAL ISSUES}

Around the turn of the century, debate was going on as to whether the new genetics would revolutionize health care, or whether an evolutionary perspective should be adopted, in which current service provision forms the basis for new or additional services. ${ }^{178,179}$ In the United Kingdom, for instance, the government White Paper 'Our inheritance, our future: realising the potential of genetics in the NHS ${ }^{180}$ built on the current system of referral via primary care to specialized clinical genetic services. ${ }^{25}$ Calnan et al ${ }^{181}$ state, however, 'increases in demand will have implications for access and equity and any serious attempts to integrate genetics into everyday clinical practice will require major changes in training and clinical practice'. In recent years, a sense of urgency has developed about issues concerning the integration of genome-based information into health care. ${ }^{6,32,76,82,182,183}$ In Europe, the PHGEN (http://www.phgen.eu) has been established to 'promote the responsible and effective translation of genome-based knowledge and technologies into public policy and health services for the benefit of population health'. In 2009, the UK House of Lords Science and Technology Committee published a report on Genomic Medicine ${ }^{184}$ calling for a new White Paper, since developments in genomic medicine moving away from a focus on single-gene disorders to include common disorders have implications for health care.

\section{Settings relevant for implementation of testing and screening in common disorders}

Within the health-care settings of many European countries, clinical geneticists, genetic counsellors, and genetic nurses have a well-defined task in diagnosing and/or counselling patients with rare genetic disorders and their family members. Recommendations for co-operation 
with other medical specialties and primary care are available, offering a framework for patient care and referral in daily practice, although referral is not always optimal. ${ }^{185}$

Although in many countries clinical genetics has been able to create a visible and viable position, staff and resources are limited. This will have an effect on the ability of current clinical genetic services to absorb large new patient groups should susceptibility testing for common disorders be proven to be viable.

Depending on the type of disorder, the organization of health care, cultures of cooperation, and factors such as scale or population density in certain regions or countries, genetic information may be incorporated into existing practices. Other circumstances may favor the setting up of intermediary or overarching organizations to support individual health professionals in using genetic information.

Moving away from clinical genetics to other medical settings. To some extent, for certain forms of genetic testing and screening, settings other than clinical genetics are already functioning, and communication and division of labor between these settings and clinical genetics are wellestablished.

For instance, in newborn population screening programs, the blood test is performed on babies in the maternity wards of hospitals or at home, the lab results are communicated to pediatricians, and the clinical geneticist would only be involved for certain types of disorders. However, from a clinical genetics point of view, it is not always clear whether counselling and informing patients in the pediatric setting is adequate. Newborn screening is a genetic screening program organized as a public health intervention program.

For clinical care regarding monogenic subforms of common disorders, an example is provided by the diagnosis and treatment for breast cancer. Germline BRCA1 and BRCA2 as well as HER2 testing in tumors has been implemented in regular health care provided by oncologists in cooperation with clinical geneticists (as stated in the section 'Testing and screening for common disorders in Europe').

The setting up of registries for monogenic subforms of common disorders provides an example of the formation of a new type of organization that supports and optimizes current division of labor between different health-care settings and that integrates genetic testing in current health-care practices. Practical circumstances related to scale and patterns of cooperation, as well as legal impediments such as privacy regulations, may hinder the formation of registries in some countries.

For common disorders, primary care, most notably family doctors, have an important role in selecting and treating people who might be at risk. For instance, in primary care, for hypertension or measuring cholesterol, tests are provided and patients are monitored. In recent years, a stronger focus on prevention in primary care has been suggested in order to tackle the expected rising disease burden caused by common chronic disorders in an aging population. ${ }^{186}$ To stimulate prevention, special consultation offered by trained personnel, such as nurse practitioners or physician assistants, may be integrated into primary health-care services. ${ }^{148}$ Genetic testing or screening for common disorders such as diabetes and coronary artery disease might be added to the existing procedures and a close cooperation between primary care and specialist care could be set up or intensified, when necessary.

Moving from traditional medical to commercial direct-to-consumer settings. In recent years it has become possible to obtain genetic tests for specific disorders in a commercial direct-to-consumer setting (DTC). ${ }^{187-189}$ Three different routes for direct-to-consumer tests may be distinguished. On the one hand tests are sold over the counter, for instance by pharmacies. Another form is presented by tests available on the internet. The client can obtain information on the website about the aim of testing and the meaning of the test result. For some tests sold on the internet, forms of counselling or contact with a physician are offered, ${ }^{190}$ for instance by phone or email. Other tests may be marketed via alternative health-care providers and consumer outlets such as sports centers and high street stores.

Especially in the United States, but also in European countries, commercial laboratories or companies offer tests on the internet, and the number of providers and tests is rising rapidly ${ }^{191,192}$ (see also Table 5 in Appendix E for a list of companies offering direct-toconsumer genetic testing). The tests range from single-gene tests for monogenic disorders or monogenic subsets of common disorders, such as BRCA1 and BRCA2, one or several SNPs or genetic variants found to be associated with common disorders such as diabetes, genomic profiles combining gene variants or SNPs, or genome-wide scans for a particular range of conditions such as cancer or cardiovascular problems, ${ }^{193}$ but which may also be used to learn more about, for instance, ancestry or traits. ${ }^{187,194}$ Even a test based on wholegenome-sequencing techniques can be commercially obtained, which is costly, although prices are dropping. ${ }^{195}$

A major problem with DTC tests is premature marketing. ${ }^{48,187,194,196,197}$ Research results suggesting associations between gene variants and common disorders are sometimes immediately used to develop and offer a test. As discussed in the section 'Common diseases', information on analytic validity alone is insufficient to assess the usefulness and performance of a test in medical services. Especially in case of common multifactorial disorders, the predictive value of gene variants or sets of gene variants will be low. By commercial valorization of research findings outside the medical domain, the established procedures for safeguarding sound assessment and establishing utility and cost effectiveness are circumvented.

Sometimes companies state that the medical and DNA data will be used for research, and in some cases clients are stimulated to share their experiences with other users. Knowing one's genome becomes part of a 'recreational' genomics. ${ }^{48}$ The boundaries between the research, the medical, and the recreational domain become blurred. ${ }^{198}$ In this way commercial initiatives may bypass not only the services of health professionals, but also the carefully established ethical standards developed for the medical domain, considering potentially psychologically and socially harmful disclosure of knowledge about one's genetic make-up. Also, the concept of confidentiality, implied in meticulous consent procedures in medical practice and biomedical research, may become overhauled. We can only learn how individuals will deal with diverging types of genetic information with experience over time. Especially in case of the profiling services, it is possible that groups of active 'early adopters' 48 will develop ways of dealing with this kind of information and pave the way for a larger audience interested in knowing more about their genetic constitution.

As far as blurring boundaries are concerned, it should also be noted that the commercial sphere should not be regarded as an isolated dimension. For instance, spin-off companies often have their roots in academia; some researchers are active both in a commercial and in an academic setting; sometimes research, also in academia, is partially paid for by industry; and also health-care professionals may be involved in commercial companies. Moreover, data sharing between commercial companies, academia, and health-care institutions has become an interesting way of increasing research potential, which stresses the need to further address issues of informed consent and confidentiality. ${ }^{198}$ 
Some of the problems with DTC tests relate to the role of the medical professional. It is unclear whether patients receive adequate counselling either before or after performing the test, whether they understand the test results correctly, ${ }^{189}$ and how the specific test relates to the overall health status of a person and his or her other health problems. Physicians claim that the interpretation of test results, and combining various forms of health information over a longer period of time, always requires a medical professional. The fact that some commercial companies include a counselling session or referral to a physician only partly tackles this objection. ${ }^{199}$ Problems might occur, for instance, regarding documenting the information in the patient's file, or because of the possibility of bias on the part of the physician who is paid by the company that sells the tests.

In addition, providing genetic services outside the traditional domain of medical genetics departments may challenge the public health-care system. DTC services may stimulate consumers to visit health-care professionals after an assessment of their risk of developing a disorder. ${ }^{200,201}$ Almost all DTC companies state in their disclaimers that the information they provide is for informational purposes only and that consumers should contact their physicians to act upon the test result. ${ }^{202}$ Therefore, there is a potential risk of overconsumption of health-care services.

Moreover, consumer genetics are organized in such a way that there is no control over the origin of the samples being analyzed. Most companies send mouth swab kits as these are easier and more practical than having to send the client to a clinic where blood is drawn. As the mouth swab is done in the privacy of the client's home, there is no way of controlling for the identity of the sample provider. Testing of third parties, such as incompetent minors or incapacitated adults, becomes possible and rather impossible to control. ${ }^{203}$ In addition, it has been shown that some DTC companies test minors without specific ethical concerns. ${ }^{204}$

In the United Kingdom, the growing offer of commercial genetic testing has been the subject of attention for the Human Genetics Commission, who issued the report Genes Direct in $2003 .{ }^{205}$ A followup of this report and revised recommendations were published in 2007: More Genes Direct. ${ }^{190}$

The recommendations in this report relate a.o. to the evaluation of the evidence used by manufacturers in the marketing of these tests. This plays a part both in the process of pre-market review and licensing procedures before the test comes to market and in the subsequent advertising and promotion of the tests and testing services. In 2003, as well as in 2007, it was underscored that certain genetic tests should only be offered by a suitably qualified health professional.

In 2007, the American Society of Human Genetics issued recommendations regarding direct-to-consumer genetic testing. ${ }^{206}$ They include the provision of adequate information on test characteristics, the strength of scientific evidence, limitations to the claimed benefits of testing, such as other factors involved in developing the disorder, and the psychological risks. Tests should have adequate clinical validity, and claims in advertisements should be truthful. It is recommended that more research is needed regarding the impact of this type of testing on consumers.

In Europe, the currently available pre-market evaluation procedure does not cover genetic tests adequately (see also the section 'Regulatory issues'). Commercial tests are subject to certain forms of quality control and lab standards, and some degree of self-regulation exists. However, especially in the case of internet offers, it is not clear how this - internationally operating - sector can be or should be regulated. ${ }^{188}$ Recently, more pleas for self-regulation or codes of conduct have been voiced. ${ }^{207,208}$ In 2009, the UK Human Genetics Commis- sion proposed a common framework of principles for direct-toconsumer genetic testing services. ${ }^{209}$ It was accompanied by a public consultation to elicit comments and enable establishing priorities in governing direct-to-consumer testing that can be supported by stakeholders both in the United Kingdom and in other countries. One of the aims was to lay the foundation for an international code of practice for professionals and companies offering DTC tests.

In order to organize and fund pre-market assessment, a combined effort of stakeholders is recommended. The European Diagnostic Manufacturers Association may serve as an example of self-organization (or function as a platform) to unite the industry and stimulate interaction with regulatory bodies in drawing new guidelines. This kind of initiative might stimulate ways to reconcile the drive for valorization of research findings with responsible entrepreneurship.

For the European context, it is important to find a balance between protecting citizens against potentially harmful tests on the one hand, and the right of citizens to obtain knowledge about their health status and genetic constitution on the other hand. This balance might be of a different character for Europe and the individual countries concerned, than for the United States.

In the Netherlands, the Health Council issued a report on screening in which the role of the government regarding commercial genetic tests was discussed. Government responsibility was not seen to be limited to tests that - because of proven benefit for public health would merit integration into an official population screening program. ${ }^{210}$ It was advised to organize the development of an official quality marking, endorsed by stakeholders from industry, professionals, and governmental bodies. Information regarding the quality of tests, as well as the pros and cons of testing, should be made available to the public, for instance through an official website. Furthermore, it was acknowledged that people should be free to order commercial tests that are found not to be harmful, if people pay for them themselves.

The role of patient organizations in commercial testing requires special attention. There is a potential danger in close connections between patient advocacy groups and industrial organizations that want to promote a certain test or medicine. On the other hand, direct-to-consumer test offers are seen by some as a means of patient empowerment. Patient advocacy groups can play a mediating role in offering accessible information on disorders and tests and allowing people to share their experience with testing and living with test results.

A specific subset of commercial genetic tests and services is offered in the field of nutrigenomics. It is quite a young and complex field, and applications are not yet clear, although claims sometimes already suggest practical benefits. The European Nutrigenomics Organisation describes nutrigenomics as 'the science that examines the response of individuals to food compounds using post-genomic and related technologies... The long-term aim of nutrigenomics is to understand how the whole body responds to real foods using an integrated approach termed "systems biology".'211

A GeneWatch report questions both the scientific claims about individual biological differences between people and their response to food, as well as the social and political implications of individualizing and commercializing diet advice. 'The idea of tailoring diets to genetic make-up is based on a false and outdated view of the role of genes. For most common diseases in most people, an individual's risk is not predictable, because multiple environmental and biological factors interact.' Companies 'are marketing misleading and inaccurate interpretations of people's genes and what they mean for their health. As this industry expands and provides multiple and conflicting dietary advice and products, there is significant potential to confuse and 
undermine healthy-eating messages. Some people may be falsely reassured that they are not at risk of particular diseases, with serious consequences for their health. New 'value-added' products such as functional foods are expensive and unnecessary....212

\section{Connections between sciences, medicine, and health care}

In reflecting on the wealth of new research data, Khoury et al ${ }^{213}$ stress the need for a sustained translational research effort in order to accelerate the integration of human genome discoveries into health care and disease prevention. Several phases of translational research, including clinical trials, guideline development, and dissemination research, are needed to ensure a safe and effective implementation. On the other hand, as this section makes clear, many research results are applied prematurely in a commercial setting, without establishing clinical validity and utility. Thus, for the testing of several common multifactorial disorders as well as for gaining insight into psychological consequences and the effect of interventions such as lifestyle modifications, a new sort of social experiment has started: learning by doing is partly replacing learning by trials.

When concentrating on the health-care setting, it is relevant to picture the wider societal and cultural framework of newly emerging technological developments, such as genetic tests. This framework enables and constrains the possibilities for innovations, and helps to identify some of the actors and factors relevant for the actual translation research as proposed by Khoury.

For the successful implementation of a new technological option such as a genetic test or genetic screening program - attunement between stakeholders on various issues concerning the new technology is necessary. ${ }^{6,214-216}$ These issues concern the test performance, the organization and funding of facilities and services, the prospective users and the demand, the economic evaluation of the testing, as well as the political and cultural acceptability. This attunement takes place on and transgresses various levels: the micro-level (the sociotechnical niche) of a specific lab or clinic; the meso-level (the sociotechnical regime) formed by already established technologies, practices, suppliers, users, rules, and values; and the macro-level (the sociotechnical landscape), the relatively stable environment of institutions, material infrastructure, and economic, social, political, legal, cultural and demographic relationships. Achterberg et al ${ }^{214}$ distinguish four types of actors or stakeholders: scientists in laboratories and clinics; regulatory, advisory, and governmental agencies; physicians, and professionals in (public) health care; and patients and their organizations.

This approach offers a tool to understand what kind of interactions retrospectively have been, or prospectively need to be - put in place before implementation of certain kinds of testing or screening is possible. It may be used to identify stakeholders and actively engage them in setting up systems of self-regulation or inviting them to establish a code of practice in relation to the use of genetic tests. For instance, an active group of patients might articulate a demand for certain kinds of screening. In the absence of such a demand, the incentive for screening needs to rely more on, for instance, active physicians, an alert government, or a strong public health authority. These features would differ not only per disorder but also per country. This approach can also be used to understand why certain new forms of screening take a long time to get implemented. For instance, in many countries there is no regime for preconceptional care, so new preconceptional genetic screening cannot be easily added to existing practices, whereas in the case of certain forms of prenatal screening current practices can be quite easily expanded. ${ }^{217}$

Picturing the wider societal and cultural framework of new genetic technologies may also be helpful in devising scenarios. ${ }^{218,219}$ For example, a scenario for dealing with an increasing offer of and demand for commercial genetic testing might be that clients inform their family doctors about the test results and that commercial tests will be integrated into an already existing entrusted doctor-patient relationship. In this view, commercialization is seen as a means of giving the patient more control over his or her health status, making them 'copractitioners' or 'comanagers of their health,'220 and thus as patient empowerment. Another scenario would be that commercial testing expands alongside the official health-care system. People who can afford - or are anxious enough - to test, may establish a wellinformed, partly highly anxious group, the 'worried well'. These people might pose an extra burden on the existing health-care system because of frequent questions concerning follow-up of test results. Health inequalities might be intensified. ${ }^{221}$

\section{Ethical and social issues}

Impact of genetic testing and screening for common disorders. It might be argued that the ethical and social issues of testing for genetic susceptibility for common multifactorial disorders differ from the issues of testing for monogenetic disorders, including monogenic subforms of common disorders. Janssens and Khoury ${ }^{222}$ state that for multiple genetic testing (genomic profiling) the differences 'lie in the lower predictive value of the test results, the pleiotropic effects of susceptibility genes, and the low inheritance of genomic profiles. For these reasons, genomic profiling may be more similar to non-genetic tests than to predictive tests for monogenic diseases.' In this line of reasoning, issues such as psychological burden, anxiety because of disclosure to family members, and problems with insurance could have a less prominent role in susceptibility testing and screening for common multifactorial disorders in comparison with testing and screening for monogenic disorders, including monogenic subforms of common disorders.

For genetic testing in relation to monogenic disorders, including monogenic subforms of common disorders and cancer in recent years, an impressive amount of health-psychological literature on risk perception, stress after testing, fatalism, the impact on family relations, etc, has been produced. ${ }^{223-227}$ However, not much social scientific research has been conducted on testing for common disorders yet. In a 2007 study from the United Kingdom, interviews with patients who had had a genetic test for thrombophilia suggested that understanding of the test depended on social background. ${ }^{228}$ As far as the influence of testing on behavior is concerned, Pijl et al ${ }^{229}$ found a modest effect when communicating familial risk (as a surrogate for genetic testing) in case of diabetes. People perceived greater control over preventing diabetes and reported to have eaten more healthily after being informed about their familial risk and the need for a healthy lifestyle compared with a control group of people who had only been informed about the need to maintain a healthy lifestyle. Otherwise, studies on testing for $\mathrm{FH}$ and hemochromatosis might give an indication of relevant issues.

In Australia, secondary-school students between 16 and 18 years of age and their teachers and parents had a positive attitude toward screening for $\mathrm{HH}$, were it to be offered to the school population. ${ }^{230}$ Yet, positive attitudes may not directly correspond to taking a test when it would really be offered. Furthermore, it is not clear how knowing one's carrier status will be experienced over the years. People might remember the meaning of the test results incorrectly, and change their lifestyles inappropriately. Also, people might simply forget the results, which makes it important that some form of registration is possible, for instance in the primary-care setting. A German pilot study in which screening for hemochromatosis was 
offered to clients of a health insurance organization concluded that screening on the basis of a test for the C282Y mutation was generally accepted and was perceived as beneficial, although uptake was modest. Reported negative psychological consequences were rare. ${ }^{131}$ In 2007, a study was published on a subset of participants in the Hemochromatosis and Iron Overload Screening Study. In comparing participants from Canada and the United States, evidence was found of at least one element of negative emotional response to genetic testing, although it was not serious enough to affect individuals' mental or physical health. Fewer Canadians than Americans were found to experience a negative response. ${ }^{231}$ A recent systematic review reported few negative psychosocial outcomes for DNA testing for $\mathrm{HH}$ in at-risk individuals, although evidence was found to be limited. ${ }^{232}$

For $\mathrm{FH}$, the perception of parents of the test results of newborn screening for $\mathrm{FH}$ has been studied. Parents perceived the condition of their child as more threatening and uncontrollable when FH was seen as a genetic condition than when it was seen as a cholesterol problem. From this the authors conclude that DNA analysis might result in a sense of fatalism, adversely affecting motivation to change behavior. ${ }^{233}$ However, in a study in which the quality of life of $\mathrm{FH}$ patients was measured up to 18 months after receiving the genetic test result, no important adverse effects on the quality of life were found. ${ }^{234}$ In a recent study on preventive behavior, Claassen et $a l^{235}$ found that genetic information on $\mathrm{FH}$ had less consequences for changing lifestyle than having a positive family history. This was not due to fatalism: almost all respondents used medication to prevent cardiovascular problems. This is in accordance with findings that information that a condition is caused by a genetic predisposition may reduce the expectation that a behavioral means of coping, such as changing diet, will be effective, but increase the expectation that medication will be effective. ${ }^{236}$

While ethical, social, and psychological issues regarding testing and screening for low-risk alleles in common disorders might be of a different character than testing for monogenic disorders, the examples above suggest that they do merit attention. In some cases misleading or misinterpreted genomic information may cause harm. Recently, scientists have warned against the commercial marketing of, for instance, susceptibility testing for schizophrenia and bipolar disorder. ${ }^{237}$ Another reason for concern stems from scientific research. The pleiotropic effect implies less direct or predictable effect from a genetic mutation and might connect preventable disorders with disorders for which no treatment is available. For example, in CVD, polymorphic variants in $A P O E$ have been found to be associated with variations in blood levels of lipids, lipoproteins, and apolipoproteins. Testing for APOE variants may yield information about the risk of developing CVD. However, one variant of APOE, the e4 allele, has also been associated with an increased risk of Alzheimer's disease. ${ }^{238}$ Future biomedical research is likely to come up with many more instances, which might make the use of testing for low-risk alleles in everyday medical-care settings more complicated than adding just another risk factor.

The 2009 UK House of Lords Science and Technology Committee report 'Genomic medicine' recommends that the UK Human Genetics Commission 'should promote a wide-ranging debate on the ethical and social issues relating to genetic tests and genetically complex diseases with the aim of improving the public understanding of genetic risk and predictive testing in common complex disorders.' ${ }^{\text {'184 }}$

Counselling. For monogenic disorders, counselling procedures and adequate provision of information have been studied extensively. It is not clear yet what type of counselling susceptibility testing would require, and what would be possible given the limited time frame of consultations in primary care. ${ }^{239}$ In the recommendations issued by EuroGentest regarding 'susceptibility testing' (sometimes referred to as risk profiling'), it was stressed that 'if the test is or is claimed to be capable of detecting high relative risk for a serious condition and thus has significant implications for risk assessment, treatment or prevention in a person or his/her near relatives, then pre- and post-test genetic counselling is needed.23 Also, the Council of Europe's Additional Protocol to the Convention on Human Rights and Biomedicine, concerning Genetic Testing for Health Purposes, ${ }^{240}$ states that counselling could vary in form and extent in accordance, in particular, with the implications of the test and their significance for the person concerned or the members of his or her family. ${ }^{241}$

In addition to (and perhaps in some cases partly replacing) counselling, high-quality information can support decision making by patients on genetic testing services. In the United Kingdom, in the DISCERN project, an elaborate scheme has been devised to assess patient information on the basis of criteria applicable to various forms of genetic disorders, ${ }^{242}$ which could also be used for testing or screening for common disorders. ${ }^{243}$ Easily accessible and high-quality information may also be of great importance in testing for common disorders. Websites and organizations currently involved in providing this kind of information in relation to monogenic disorders, such as patient advocacy groups, might extend their services to include information on common complex disorders.

Legal issues. Regarding insurances, again we may turn to screening for $\mathrm{FH}$ as an example. In the Netherlands, a few patients diagnosed with $\mathrm{FH}$ did meet difficulties in finding life insurances. ${ }^{244}$ Active involvement of insurance companies with patient groups resulted in establishing guidelines for offering insurance to $\mathrm{FH}$ patients. ${ }^{245}$ In the United Kingdom, since 2001 there is a voluntary moratorium on the use of most genetic test results by insurers, which has been extended to $2014 .^{246}$ It is a general concern that fear of social and economic drawbacks might withhold people from obtaining information about their genetic constitution for health purposes. Genetic non-discrimination legislation would be important in creating favorable conditions for genetic testing and screening. Preferably, stakeholders from insurance and other relevant sectors can be stimulated to find common ground to ensure that both employers and insurance companies use genetic information in a responsible and ethically justified manner.

Should clinically useful genetic tests for common disorders be identified, an adequate system of reimbursement for testing and treatment, and preferably also for prevention, by means of collective health insurance is vital to ensure that individuals are not excluded from health care as a result of high genetic risk. In this way, the danger of furthering inequalities in health care as a consequence of increasing genetic knowledge may be reduced.

Concerning the marketing of tests for common disorders, many regulatory issues will need to be addressed. This issue will be dealt with in the next section.

Research and development of new test devices are costly. Patenting may be a way to ensure return on investment; however, research may be hampered by legal restrictions on use and dissemination of genetic knowledge. A concerted action between stakeholders in industry, health care, professional and patient organizations, and governmental bodies is necessary to balance the interests of industry with the requirements of furthering the availability of testing devices in a responsible manner (see also the ESHG Background Document ${ }^{247}$ and Recommendations ${ }^{248}$ concerning patenting and licensing in genetic testing). 
When genetic testing or screening would become more prominent in health-care services for common disorders, current facilities for storage would be stretched. Storage of samples or tissue and information on health and lifestyle of individuals in biobanks may be useful for research. Legislation is necessary to secure privacy and nondiscrimination regarding the use of genetic information to ensure an enduring public trust.

Confidence: public understanding and participation. In the literature on public understanding of technology, since the 1970s the idea of a knowledge gap between public and science has been challenged. On the one hand, laypeople and patients do have a distinct and often relevant form of prior (local, practical) knowledge to which professional knowledge ought to relate. ${ }^{249}$ On the other hand, as recent health psychological research has shown, increasing knowledge does not always lead to greater acceptance of new technology. In sociology and social studies of science, this has prompted the notion of 'upstream engagement' of the public voice and the need for professionals to enter into dialogue with the public instead of unilaterally 'explaining' new technologies. Entering into dialogue, public engagement, and public consultation is not only seen as essential elements in gaining knowledge about the public's perception on and acceptance of new innovations, but are also seen as central elements of the functioning of modern democracy. ${ }^{250,251}$ Perhaps in the United Kingdom, public engagement has found a more firm platform and official status than in other European countries in the aftermath of public scandals concerning BSE and GM foods, which were seen to undermine public confidence in science and technology.

In the United Kingdom, new initiatives have been taken to enter into dialogue with the public at large. The Human Genetics Commission has set up various forms of public consultation - for instance, on the supply of genetic tests directly to the consumer. ${ }^{190,205}$ Also, in the United Kingdom six genetic knowledge parks were active in the field of public engagement. ${ }^{250,252}$ For the Netherlands, the Centre for Society and Genomics has a similar mission of stimulating dialogue. ${ }^{253}$ To enable citizens to reflect on biomedical issues such as genetic testing, genetic literacy needs to be stimulated, for instance by improving secondary education in life sciences. ${ }^{254}$

Besides on a collective level, confidence has an important role in the individual doctor-patient relationship. In medical curricula and health psychology, a similar trend toward taking the patient more seriously can be discerned. In recent years there has been a growing experience with the implementation of forms of communication between doctors and patients, such as shared decision making and informed decision making, that are highly relevant when discussing genetic testing options. These techniques are well known in clinical genetics practice. Clinical geneticists may act as resource persons or assist in education of other health-care professionals to raise the standards of these forms of decision making and counselling applied by these professionals.

Training. Although there is growing evidence that genomics will change the practice of medicine, the question remains: how. Currently, health-care providers have deficient knowledge about genetics. ${ }^{147}$ For primary care especially, basic knowledge is necessary to be able to identify patients who require further investigation or referral to a specialist, and to make communication possible between primary-care professionals and specialists. Primary-care professionals should also be able to understand inheritance patterns and communicate this information to patients in order to counsel them on issues of risk, testing, and familial aspects. ${ }^{32}$
In preparing health-care professionals and students for the emerging possibilities, given the rapid developments in genomic science, it is impossible to provide a final base of knowledge. Rather, we must find ways to impart knowledge, skills, and attitudes that will make students lifelong learners of genetics and genomics. ${ }^{32}$ In addition, transparent communication and cooperation structures with relevant other health-care providers and genetic specialists are relevant to enable information sharing, referral, and monitoring.

In a EuroGentest Network project, contacts were established with a.o. the ESHG Education Committee, resulting in several initiatives to raise the level of education and information regarding genetics among health professionals. A working group was set up to identify minimum levels of competencies for health-care providers in Europe. ${ }^{255} \mathrm{~A}$ website was established giving information on courses and offering hyperlinks to national professional groups and organizations. ${ }^{256}$

Developing countries. Developing countries are a heterogeneous category of countries in which $80 \%$ of the world population live and are characterized, among many other things, by medium-low incomes, high degree of economic and social inequality, a high load of preventable diseases of poverty (infectious and nutritional diseases), and a rising prevalence of non-communicable diseases, which have already become the main sources of morbidity and mortality in most developing nations. Health systems in developing countries are characterized by fragmentation and under-funding of the public sector, coexisting with a small private sector that caters to the wealthy elites with similar levels of spending and high-technology approaches to health that their equivalents enjoy in developed countries. ${ }^{257}$ In most developing countries, the total annual per capita health expenditure rarely surpasses USD 300, with appalling inequities in access according to social class. There is no tradition of government oversight of the quality of health care, and much less of quality of laboratory testing.

All the considerations, caveats, and skepticism detailed in this report on the relevance, priority, and appropriateness of genetic susceptibility testing or screening for common disorders in Europe apply as well to developing countries. Furthermore, and because of economic and cultural factors, among the latter the priorities for the control and prevention of common complex disorders should emphasize 'generic' population-wide programs of proven public health impact, such as tobacco control, healthy nutrition, physical activity, stress reduction, and avoidance of exposure to environmental mutagens, rather than 'individualized medicine' based on genetic susceptibility testing. In some special cases, it could be that the prevention of rare monogenic subtypes of common diseases, such as BRCA1 and BRCA2 mutations causing breast and ovarian cancer, FH, FAP, and others that may come in the future, may benefit from cascade predictive testing in families when the approach is justified by their population prevalence and where the detection of an index case allows the proper assessment of the genetic risk in the family. Even within this scenario, the utility of the information gained has to be assessed before use. Furthermore, one would have to carefully weigh the relative cost/ efficiency of different available preventive options carefully, as well as consider the possible detrimental effect of common problems in developing countries, such as lack of health personnel qualified for genetic counselling and testing, genetic stigmatization and discrimination, over-reliance on technology, lack of qualified governmental oversight and quality control, and lack of a public health system that can sustain the approach.

For the prevention and control of common complex disorders in developing countries, much is to be gained by raising the standards of living conditions (nutrition, avoidance of deleterious environmental 
agents) and access to primary health care. Given the scarcity of resources and multiple other priorities, the proof of clinical validity and utility of genetic testing for common disorders in promoting health with equity and justice is of even more ethical and economic importance in developing than in developed countries. These principles should apply as much to the general population of developing countries as well as to the wealthy minority that make use of private health care, because in one way or another the expenditure in private health care tends to drain resources from the overall society. Thus, regulations and guidelines for the use of genetic testing based on the evidence of clinical validity and utility are a responsibility that governments should take seriously to protect their citizens from the untimely and improper use of unsafe or inefficient predictive genetic technologies.

Given that globally most research funds come from the for-profit private industry of developed countries, it is no surprise that diseases that affect the Western world have received more attention in research. The lack of development of drugs against scourges particularly affecting the poor in developing as well as developed countries (tuberculosis, malaria, Chagas disease, HIV, and many others) can only be explained by the low interest of the industry in drugs whose market would be primarily among the poor who cannot afford the prices of those products. Pharmacogenetic approaches, however, are now been heralded as a tool to enhance therapeutic efficiency qand reduce the side effects of some drugs (for example, the metabolism of the antituberculosis agent isoniazide depends on variability in the NAT2 polymorphism). Pharmacogenetic testing should be subjected to high scrutiny, in the same way that this report advocates for susceptibility testing in general, and these principles should apply to developing countries as much as to developed countries. Studies on the genomes of pathogenic organisms have furthermore contributed to the development of therapy, for instance in the prevention and therapy of malaria, where the D-xylulose 5-phosphate pathway can be inhibited by the antibiotic fosmidomycin. ${ }^{258}$ Techniques used in genetic and genomic research are also used to develop vaccines.

As a final comment in this section, it should be cautioned that there is not a single developing country in which the health situation, economic, social, and cultural factors are exactly alike any other country. Therefore, in order to be successful, approaches to prevent and control common disorders should take into account all these factors (see also Christianson \& Modell $^{259}$ ).

\section{REGULATORY ISSUES}

Genetic tests fall under the broader statutory regime for the regulation of in vitro diagnostic (IVD) medical devices. This regime is risk-based, so the degree of regulatory oversight is contingent on the risk classification of the device. The regime for medical devices is concerned with ensuring the safety and effectiveness of medical devices and its most powerful tool is design dossier assessment, which used to assess, prior to the marketing of a medical device, whether the manufacturer has met the regulatory requirements, including clinical evaluation to support the intended use of the product as set out on the product label and in any promotional material.

Ensuring truth-in-labelling and truthful promotion - an honest account of the strengths and weakness of a test's performance - can be thought of as one of the fundamental functions of statutory premarket review of devices. For high-risk tests, regulators may set out in some detail the types of clinical studies that will be required to gain pre-market approval. Once a device is on the market, it is subject to post-marketing surveillance and, if necessary, regulators will remove unsafe products from the market.
Regulation of IVD devices controls the provision of information on the performance of a device by setting requirements for the data that are to appear in:

- the technical documentation;

- the label;

- the packaging and any promotional material.

The technical documentation contains the data that may be reviewed by the regulator; the label contains the performance data required by the users of the test and any necessary instructions for the safe and proper use of the test. Claims made for the stated intended use must be substantiated by adequate performance evaluation data.

This chapter draws on work by Stuart Hogarth and David Melzer in collaboration with members of EuroGentest, in particular the outcomes of a meeting about the IVD Directive held by EuroGentest in 2007 and a briefing on the Directive that Hogarth and Melzer prepared following that meeting. This briefing has been presented to the regulatory authorities of EU member states, has been adopted as a EuroGentest report, and will inform future EuroGentest work on the Directive. Further analysis of these regulatory issues is consequently being pursued by the legal working group within the Public Health Genomics European Network (PHGEN). ${ }^{7}$ They are assessing the current regulatory state of the art in Europe and, furthermore, trying to define areas for harmonization.

\section{The IVD Directive in the context of genetic testing}

The European in-vitro diagnostic medical devices directive (Directive $98 / 79 / \mathrm{EC})^{260}$ and the measures transposing it into national law constitute the main regulatory framework for genetic tests marketed in Europe. The Directive regulates the placing on the market and the putting into service of in-vitro diagnostic medical devices with the aim of ensuring their safety and performance for patients and users.

The Directive's scope includes genetic tests, as they are considered to be IVD medical devices. But, genetic tests that do not have a medical purpose, for example, genetic tests for forensic purposes, are not covered by the Directive.

In principle, the Directive contains all essential requirements for genetic tests, but it covers the product, not the broader aspects of testing (how testing, as a service, should be regulated - eg, provision of counselling), as the provision of health-care services is under the control of individual Member States.

Some issues that might need further clarification in the regulatory framework of genetic tests under the Directive are discussed in the sections that follow.

In-house tests. It is recognized that a number of genetic tests are socalled 'in-house' tests. Clarification is required on which in-house tests are covered by the Directive. Tests that are 'manufactured and used only within the same health institution', are excluded from the scope of the current Directive. These in-house tests are covered by national rather than harmonized community legislation. However, the term 'health institution' needs elaboration or definition to clarify that commercial laboratories are covered by the Directive. Currently it is believed that many commercial test laboratories wrongly claim to be 'health institutions' and thus claim to be excluded from the Directive.

There are many open questions around this 'in-house' concept:

1. Is there a harmonized interpretation in all Member States?

2. Definition of professional and commercial contexts.

3. What is a 'health institution'? Can laboratories be health institutions? 
Risk classification. The primary reason that most genetic tests are not subject to independent pre-market review in the EU is that they are either not listed in Annex II list A of the Directive 98/79/EC or are not self-tests and therefore the manufacturer is not required to submit their technical documentation to a notified body. An international comparison of device regulations shows that the European approach is unique. In the United States, Canada, and Australia, genetic tests that fall within the medical device regulations are all treated as moderate to high risk - and so are generally subject to pre-market review (in Australia, some genetic tests are Class II and exempt from pre-market review). There are a number of reasons for considering that many genetic tests are moderate to high risk:

1. They are often stand-alone, with no confirmatory test available.

2. They are used for critical clinical purposes, such as for pre-implantation genetic diagnosis and selecting treatments (pharmacogenetics).

3. They may have a serious psychological impact (eg, Huntington's disease).

4. Many new tests are highly complex, involving multiple alleles or multiple genes, making interpretation more difficult.

5. If it is a test that is performed in a single reference laboratory, then it will not undergo informal peer review by the pathology community.

6. New genetic tests carry the risks associated with all novel devices unproven performance in the field and lack of familiarity on the part of users.

'A major problem identified with the current approach in the Directive is an apparent lack of consistency in the risk classification of the products. ${ }^{3191}$ However, the Directive sets out a number of criteria to be considered when considering an amendment or an extension to Annex II:

(a) 'any relevant information available from the vigilance procedures and from external quality assessment schemes as referred to in Article 11;

(b) the following criteria:

(i) whether total reliance has to be placed on the result obtained with a given device, this result having a direct impact on subsequent medical action, and

(ii) whether action taken on the basis of an incorrect result obtained using a given device could prove to be hazardous to the patient, to a third party or to the public, in particular as a consequence of false positive or false negative results, and

(iii) whether the involvement of a notified body would be conducive to establishing the conformity of the device.'260

Furthermore, manufacturers are required to notify new products to the Competent Authorities, in accordance with Article 10(4) of Directive 98/79/EC, thus giving the Member States the opportunity to closely examine and assess any new test and, where necessary, ask for amendment or extension of Annex II.

But although there is a clear set of criteria, there appears to be little consistency regarding what is classified as moderate risk and what is low risk. Thus, Chlamydia tests are in Annex II, List B, but no other tests for sexually transmitted diseases; PSA is also on List B, but no other cancer tests, such as the Pap smear, CA125, CEA, etc; there is one heritable disorder, PKU, but no others.

It can be argued that a list-based approach to classification is not a sufficiently efficient mechanism for risk classification, particularly for novel tests, as, in order to amend or extend Annex II, the Commission must first examine the request and then propose a legislative measure. Where Member States do not introduce such a substantiated request, inconsistency can arise. For instance, Gen-Probe's PCA3 test quantifies the PCA3 mRNA in a patient's urine sample as a marker for prostate cancer and thus performs exactly the same clinical function as the PSA test, yet it has not been added to Annex II, List B, despite the fact that PSA is in Annex II, List B.

The proposed GHTF model: The risk classification schema developed by the Global Harmonisation Task Force appears to be both more comprehensive and more consistent. ${ }^{261}$ Largely modelled on the Australian system (itself a refinement of Canada's model) it is a four-class system running from high- to low-risk. The risk class of a test is assessed using a number of criteria, such as the intended use/indications for use, the skill of the user, the degree of reliance placed on the test result, and the potential impact on public health and the patient.

The GHTF model places some genetic tests into the moderate-tolow risk category (Class B) and others in the moderate-to-high risk (Class C) category. The principle seems sensible, as it is clearly the case that some genetic tests pose greater risks than others. However, when linked to the conformity assessment model, this distinction may create problems, as tests in Class B are not subject to independent premarket review.

This could be a problem for a novel Class B test. The GHTF model does not treat novelty as a risk factor. This is in contrast to the US regulatory system, which treats novelty as a risk factor - novel tests are automatically classified as Class III and subject to the most rigorous conformity assessment route. This classification can be appealed, and generally most new tests are reclassified as Class II, and subject to a less rigorous pre-market review (de novo $510 \mathrm{~K}$, roughly equivalent to the Class C conformity assessment route in the GHTF model). Experience would suggest that lack of familiarity with a new test - whether it is the testing platform, the biomarker/s, the interpretative algorithm or any combination of these three - can lead to errors. The true performance of a test, both its analytic and clinical validity, is not known until the test has been in routine clinical practice for some time. Furthermore, tests generally perform less well in routine use than they do in clinical investigations. For these reasons, novel tests are more likely to lead to incorrect results and so novelty should be formally acknowledged as a risk factor.

Analytic and clinical validity. Most stakeholders believe that the Directive requires manufacturers to provide evidence of a test's analytic validity, but only requires evidence of a test's clinical validity if clinical claims are made by the manufacturer. ${ }^{191}$ However, recent discussion with a range of competent authorities has revealed that in fact member states disagree about the scope of the Directive, with some believing that it covers both analytic and clinical validity as minimum requirements. An international comparison indicates divergence between the US and Canadian systems and those of the EU and Australia in this regard.

Clinical claims: MEDDEV guidance on Research Use Only (RUO) products issued in 2004 highlights the issue of the distinction between

\begin{tabular}{lll}
\hline Country/region & Analytic validity & Clinical validity \\
\hline USA & Yes & Yes \\
Canada & Yes & Yes \\
Europe & Yes & Unclear \\
Australia & Yes & Only if clinical claims made? \\
\hline
\end{tabular}


research and clinical use. This guidance clearly states that an IVD test with no intended medical purpose is not a test under the IVD Directive, it is simply an RUO product:

In summary for a product to be categorized as an RUO product it must have no intended medical purpose or objective. When a medical purpose has been established based on sufficient and broadly agreed upon scientific, diagnostic and clinical evidence, then the product must comply with the requirements of the Directive before the manufacturer can place it on the market with an intended IVD use. ${ }^{262}$

It could be inferred that, in order to define a medical purpose, a manufacturer would have to make a clinical claim (eg, by identifying gene $\mathrm{X}$, this test diagnoses disease $\mathrm{Y}$ ), and if they make a clinical claim then they must support it with evidence. It would seem that once a manufacturer has a stated clinical purpose for a test, then they must provide data on its clinical validity.

The intended clinical use: Finally, looking at the Directive requirements concerning safety and performance which all IVDs must comply with before bearing CE marking and being placed on the market, is it possible to fulfil the Directive's essential requirements by only providing data on a test's analytic validity? GR Higson, a UK expert on device regulation closely involved in the development of the medical devices directives, commented on this issue, stating that:

final confirmation of the safety and performance of a medical device is normally provided by observation of the behaviour of the device in its intended use with patients... Essential requirements 1 and 6 , and in some cases 3 , can only be satisfied by the evaluation of clinical data relating to the use of the device. $^{263}$

The first essential requirement states that the test must not 'compromise, directly or indirectly, the clinical condition or the safety of the patients, the safety or health of users or, where applicable, other persons'. Furthermore, it says that 'any risks which may be associated with their use must be acceptable when weighed against the benefits to the patient and be compatible with a high level of protection of health and safety. One could argue that one can only assess the benefits of a device in relation to an intended clinical purpose. Risk assessment also requires knowledge of the clinical purpose for the device. For IVD devices, one of the main risks are the clinical consequences of an incorrect result. Since such incorrect results might arise from either poor analytic validity or poor clinical validity, it would appear logical that a comprehensive risk assessment would include the evaluation of the clinical validity of the test for its intended use.

Requirement three states that 'the devices must be designed and manufactured in such a way that they are suitable for the purposes referred to in Article 1(2)(b), as specified by the manufacturer, taking account of the generally acknowledged state of the art. They must achieve the performances, in particular, where appropriate, in terms of analytical sensitivity, diagnostic sensitivity, analytical specificity, diagnostic specificity, accuracy, repeatability, reproducibility, including control of known relevant interference, and limits of detection, stated by the manufacturer ...' Common usage of the terms analytical sensitivity, diagnostic sensitivity, analytical specificity, and diagnostic specificity would lead one to understand analytical sensitivity and specificity as referring to analytic validity and diagnostic sensitivity and diagnostic specificity as referring to clinical validity. However, in the Commission Decision 2002/364/EC on common technical specifications for IVD medical devices, ${ }^{264}$ the terms 'diagnostic sensitivity' and 'analytic sensitivity' are defined thus:

(Diagnostic) sensitivity - The probability that the device gives a positive result in the presence of the target marker.

Analytical sensitivity - In the context of the CTS it may be expressed as the limit of detection: ie, the smallest amount of the target marker that can be precisely detected.

To answer the debate on clinical validity, these definitions may need to be re-visited in the future.

Predictive testing. At the April 2005 MDEG meeting, the IVD Technical Group was asked to analyze the Directive in the context of genetic testing. They produced a note on this issue for the Competent Authorities. One of its conclusions addressed the question of which genetic tests would be covered by the Directive. It stated that:

2. But, genetic tests that do not have a medical purpose, eg genetic tests for forensic or predictive purposes, are not covered by the Directive.'191

As most genetic tests can be used for both diagnostic and predictive purposes, and a significant amount of genetic testing is predictive rather than diagnostic, this conclusion may be highly significant. Predictive applications include the prediction of late-onset disorders such as Huntington's disease, risk assessment for common, complex diseases such as breast cancer and diabetes, prediction of response to therapy (pharmacogenetics), and prognostic tests such as Agendia's MammaPrint test, which predicts the risk of breast cancer recurrence.

The intent of the Technical Group's statement is unclear. Predictive tests are currently covered by the Directive, as at least one test in Annex II, List B, is predictive: 'reagents for... evaluating the risk of trisomy 21'. Furthermore, predictive tests meet the core requirements for definition of a medical device, that is, they are 'for the purpose of diagnosis, prevention, monitoring, treatment or alleviation of disease' and they also meet the definition of an IVD, that is, their purpose is 'providing information concerning a physiological or pathological state. Manufacturers of predictive tests clearly think they are covered by the Directive, as they are CE-marking them, for example, Roche Amplichip and Agendia's MammaPrint.

The IVD Technical Group recognizes that the text can be misinterpreted in this way and is currently analyzing the syntax of the sentence, as the example given in the sentence is meant to refer to genetic tests for non-medical predictive purposes.

Statutory post-marketing surveillance. Together with pre-market controls, the EU legislative framework calls for active post-marketing controls including post-market surveillance (PMS).

In general, there are three forms of post-market controls, one is for surveillance of adverse events, the second is a more general aspect of the quality assurance program that manufacturers must have in place, and the third is market approval, whereby a product (such as a medicinal product) is allowed on the market, but with additional and systematic data collection, this might include a Phase IV trial in the pharmaceutical sector.

In the past, device regulation, like the pharmaceutical legislation, has tended to focus on pre-market review, but post-marketing surveillance has taken on increasing importance in recent years. For instance, when the Office of In Vitro Diagnostics was established in the Food and Drug Administration (FDA), it united the pre-market and post-marketing departments to establish a system for controlling the total product lifecycle - from initial conception of a product through 
to its eventual obsolescence. ${ }^{265}$ In doing so, the FDA acknowledged that because performance so often varies in clinical practice, it was essential 'to take an interest in how a device really works when it enters the marketplace.'266

In Europe, the IVD Directive requires manufacturers to have a systematic procedure to review experience gained from their devices in the post-production phase. Ideally this should be part of the manufacturer's quality system. Guidance from the European Association of Notified Bodies indicates that the PMS system should be in place to collect data on issues such as 'changing performance trends [and] performance in different use populations. ${ }^{267}$ Another guidance, on clinical evaluation in the post-marketing phase, indicates the importance of what it terms post-market clinical follow-up in certain critical circumstances, which may be the severity of the disease, or the innovative nature of the technology, that is, when 'the design of the device, the material, the principles of operation, the technology, or the medical indication is new?

The PMCF plan can take the form of an extended follow-up of patients enrolled in the pre-market trials, and/or a prospective study of a representative subset of patients after the device is placed on the market. It can also take the form of open registries. ${ }^{268}$

The suggestion that the novelty of a device may be a trigger for greater emphasis on PMS is also reflected in the Directive. Articles 10 and 11 include certain obligations on manufacturers to inform competent authorities when they are introducing 'new products', that is, if 'there has been no such device continuously available on the Community market during the previous three years for the relevant analyte or other parameter' or if 'the procedure involves analytical technology not continuously used in connection with a given analyte or other parameter on the Community market during the previous three years.'

Within the recitals to the Directive that speak to these articles, particular reference is made to microarrays: '.. this is true in particular of high-density DNA probe devices (known as microchips) used in genetic screening. Such new products are subject to special vigilance procedures, whereby any time in the first 2 years after registration the competent authority 'may at any time within the following two years and on justified grounds, require the manufacturer to submit a report relating to the experience gained with the device subsequent to its being placed on the market'. There is no information available on how this provision has been used in practice.

\section{FDA regulation}

There are three relevant government institutions involved in oversight of genetic tests in the United States, all contained in the Department of Health and Human Services. The FDA oversees drug and device licensing, including diagnostic test kits and reagents. The Center for Disease Control (CDC) has a public health focus; its Division of Laboratory Services develops guidelines and policies for diagnostic testing and the Centers for Medicare and Medicaid Services administer regulatory management of laboratory services and their reimbursement.

Policy with regard to the use of genetics in health care has also been shaped substantially in recent years by the activities of the National Institutes of Health (NIH) and Department of Energy, following their investments in the Human Genome Programme. A report on the context of genetic testing in the United States, commissioned by the NIH-Department of Energy working party on ethical, legal, and social implications of human genome research in 1995 and published in 1998, highlighted the need for greater regulatory oversight of genetic testing service. Following this, in 1998 the Clinical Laboratory Improvement Advisory Committee in the CDC recommended that the Clinical Laboratory Improvements Act should be updated to establish specific regulations to address genetic testing, and in 1999 the Department of Health and Human Services Secretary's Advisory Group on Genetic Testing (SACGT - formed on the recommendations of the 1998 Holtzman and Watson report) ${ }^{269}$ in turn called for greater oversight. However, to date no new regulation has been agreed upon, although the CDC is developing new guidelines.

In this context, the CDC initiated in 2004 the EGAPP project with the objective of establishing and evaluating a systematic, evidence-based process for assessing genetic tests and other applications of genomic technology in the transition from research to clinical and public health practice. ${ }^{85}$ An EGAPP Working Group was established in 2005, and its role involves establishing methods and processes for evidence-based reviews on the evaluation of genomic applications as well as the development of guidelines. The group has commissioned evidence reviews on a number of tests and issued its first recommendations at the end of 2007 (on the use of CYP450 testing to guide the use of SSRIs in the treatment of adults with depression).

Finally, the FDA established an Office of In Vitro Diagnostic Device Evaluation and Safety ${ }^{270}$ in 2002 and since then has published several guidelines regarding IVDs. ${ }^{271}$ Most relevant to this paper's topic are the guidelines on pharmacogenetic tests and genetic tests for heritable markers (published in June 2007). ${ }^{272}$ The FDA have vacillated on the issue of whether they have the authority to regulate in-house tests; however, in the last 2 years, the agency has gradually begun to intervene on a case-by-case basis in what it now terms 'laboratorydeveloped tests. This piecemeal approach has now culminated in a new draft FDA guidance in which the agency asserts that it has the authority to regulate in-house tests as medical devices and indicates that it is now intending to exercise that authority over a class of complex tests that require interpretative algorithms to generate results. ${ }^{273}$ The forthcoming SACGHS on oversight of genetic tests recommends that FDA broaden its involvement in this area to address, in some way, all laboratory-developed tests.

According to the SACGHS, ${ }^{274}$ clinical utility for clinical decisionmaking is the balance between the benefits and harms of testing and ensuing follow-up evaluation, treatment, or prevention ( $p$ 117). Clinical utility must be evaluated within a specific context and utility may vary, depending on the context and available alternatives. The SACGHS report states: 'The additional benefit or harm that would be achieved by using the genetic test is called the incremental benefit or incremental harm. These benefits and harms should be considered at the individual, family, and societal levels'.

Claims will more typically relate to claims to quantify genetic risk. SACGHS ${ }^{274}$ notes that avoidable false-positive or false-negative results may occur if a test has not been subject to appropriate analytical validation and that significant harms (real or potential) can occur if a genetic test is used before its clinical validity is understood (pp 108111). For example: 'In the event of false-positive test results, individuals may be exposed to an unnecessary battery of testing or treatment. A false-negative test result could give false reassurance regarding risk due to nongenetic causes or induce psychological effects such as survivor guilt. False-negative results may delay diagnosis, screening, and treatment' ( $p$ 109). In order to assess clinical validity, data must be provided on sensitivity, specificity, positive predictive value, negative predictive value, etc. For multiple variants, a receiver 
operator curve can be calculated, provided the genetic architecture of the disease is known (see, eg, Clayton ${ }^{69}$ ). The area under the curve (AUC) provides a measure of the suitability of the test for use as a screening test in the general population (see, eg, Jakobsdottir et $a l^{73}$ ).

\section{OECD and clinical utility}

The recent OECD Guidelines for Quality Assurance in Molecular Genetic Testing, ${ }^{275}$ which outline the principles and best practice for genetic testing, focus primarily on analytic validity and laboratory quality assurance. However, the OECD Guidelines also state the following:

- Advertising, promotional and technical claims for molecular genetic tests and devices should accurately describe the characteristics and limitations of the tests offered (A.9).

- Laboratories should make available information on the analytical and clinical validity of tests (A.ii).

- Laboratories should make available to service users current evidence concerning the clinical validity and utility of the tests they offer (B.vi).

- The interpretation of molecular genetic test results should be appropriate to the individual patient and should be based on objective evidence (D.4).

- Reports should be timely, accurate, concise, comprehensive, and communicate all essential information to enable effective decisionmaking by patients and healthcare professionals (D.ii).

The Annotations to the OECD Guidelines (paragraphs 34 and 35) add the following:

- Information pertaining to the clinical validity and utility of a test should be based on relevant existing medical guidelines and peer reviewed literature and may include or be supplemented by inhouse studies appropriate for peer review. Sources of data should be cited.

- An assessment of who should be offered the test is part of the assessment of clinical validity.

- Measurements of validity include sensitivity, specificity, positive predictive value, and negative predictive value.

- Clinical utility refers to the anticipated effect(s) of the clinical use of the test result, including on health outcomes, recognising that a variety of factors influence this outcome.

\section{Council of Europe and clinical utility}

In 2008, the Council of Europe adopted an Additional Protocol to the Convention on Human Rights and Biomedicine, concerning Genetic Testing for Health Purposes, ${ }^{276}$ that sets down principles relating to the quality of genetic services, prior information and consent, and genetic counselling. It lays down general rules on the conduct of genetic tests, calling for measures to ensure that genetic services are of appropriate quality: a quality assurance program implemented in each laboratory; that laboratories are subject to regular monitoring; that tests meet the generally accepted criteria of scientific validity and clinical validity; and that the persons providing genetic services have appropriate qualifications to enable them to perform their role in accordance with professional obligations and standards. The specific statement on clinical utility is very clear: 'Clinical utility of a genetic test shall be an essential criterion for deciding to offer this test to a person or a group of persons.'

The protocol also deals with direct-to-consumer genetic tests, a market that seems to be growing rapidly. It specifies the conditions in which tests may be carried out on persons not able to consent. Also covered are the protection of private life and the right to information collected through genetic testing. Finally, the Protocol touches on genetic screening. The protocol was adopted in May 2007 and opened for signature in November 2009. Once signed, it will be legally binding in international law.

\section{The implications of intellectual property rights for susceptibility} testing and diagnostics

In spite of its stimulating effect on innovation, intellectual property has also been suggested to potentially inhibit research as a result of the proliferation of DNA patents, ${ }^{277}$ resulting in limited access to novel treatments and diagnostics (eg, as a result of high licensing fees). ${ }^{278}$ This argument is particularly supported by cases where patent holders make broad claims (eg, Oncomouse patent) or exert strict monopoly rights (eg, Myriad Genetic holds several patents on the breast cancer genes $B R C A 1$ and $B R C A 2$, and exerts strictly its monopoly rights, not allowing testing of the genes outside its laboratory). Further evidence in this direction is provided by a recent study indicating the negative impact of patenting and licensing practices on diagnostic research and testing carried out by clinical laboratories. ${ }^{278}$

Although some reports have argued that substantial empirical evidence to support the potentially inhibitory effects of IPR on research is still lacking, ${ }^{279}$ a wider concern with regard to diagnostic tests remains, especially as emerging techniques allow the detection of multiple mutations at a time (microarray-based tests) to diagnose disease susceptibility or drug disposition. ${ }^{280}$ In this context, the development of patent thickets (a situation where different owners have overlapping patent rights requiring multiple licenses) could pose a significant barrier. The emergence of new applications for pharmacogenomics may further complicate the situation. ${ }^{281}$

Recent evidence indicates that intellectual property rights may present a significant barrier to pharmacogenomics research and the development of related tests. ${ }^{282,283}$ The type of patents that may be issued to protect such tests would likely cover combinations of biomarkers associated with, for example, drug efficacy or response. A proliferation of such patents could create major difficulties for further development of diagnostics if the patented sequences are associated with multiple phenotypes. In this case, it would be required that several licenses are acquired prior to developing a particular test, with serious cost implications.

\section{CONFLICT OF INTEREST}

The authors declare no conflict of interest.

\section{ACKNOWLEDGEMENTS}

This work was supported by EuroGentest, an EU-FP6-supported NoE contract number 512148 (EuroGentest Unit 3: 'Clinical genetics, community genetics and public health', Workpackage 3.2). We wish to acknowledge all persons that have attended the expert workshop 'Clinical validity and utility of genetic susceptibility testing in common disorders' in Seville, 8-10 October 2007, either as participant or as speaker, or have given input to the Background Document and/or Recommendations by providing critical comments or suggestions, most notably: Jacques Beckmann, Linda Bradley, Angela Brand, Arnold Christianson, Björn Dahlbäck, Wybo Dondorp, Karl Freese, Alberto Gutierrez, Gunnar Houge, Tony Holtzman, Michael Hopkins, Alastair Kent, Mari-Lise Migueres, Irmgard Nippert, Klaus-Peter Lesch, Jan Mous, Gert-Jan van Ommen, Aurélien Perez, Dragica Radojkovic, Beverly Searle, Barbara Starfield, Birgit Verbeure, Dirk Stemerding and the PHG Foundation. The workshop was organized by Martina Cornel, Dolores Ibarreta, Ulf Kristoffersson, Jörg Schmidtke, Eleni Zika.

Members of the PPPC were: Nurten Akarsu, Pascal Borry, Anne CambonThomsen, Martina Cornel, Thoas Fioretos, Francesca Forzano, Veronica van 
Heyningen, Shirley Hodgson, György Kosztolányi, Ulf Kristoffersson, Jan Lubinski, Meral Özgüc, Christine Patch, Aad Tibben, Lisbeth Tranebjaerg, Jorge Sequeiros.

1 Visscher PM, Montgomery GW: Genome-wide association studies and human disease: from trickle to flood. JAMA 2009; 302: 2028-2029.

2 Hirschhorn JN, Lohmueller K, Byrne E, Hirschhorn K: A comprehensive review of genetic association studies. Genet Med 2002; 4: 45-61.

3 Welcome Trust Case Control Consortium: Genome-wide association study of 14000 cases of seven common diseases and 3000 shared cohorts. Nature 2007; 447: 661-678.

4 Topol EJ, Murray SS, Frazer KA: The genomics gold rush. JAMA 2007; 298: 218-221.

5 Arnett DK, Baird AE, Barkley RA et al: Relevance of genetics and genomics for prevention and treatment of cardiovascular disease: a scientific statement from the American Heart Association Council on Epidemiology and Prevention, the Stroke Council, and the Functional Genomics and Translational Biology Interdisciplinary Working Group. Circulation 2007; 115: 2878-2901.

6 Brand $A$, Brand $H$, Schulte in den Bäumen T: The impact of genetics and genomics on public health. Eur J Hum Genet 2008; 16: 5-13.

7 Public Health Genomics European Network. http://www.phgen.eu/typo3/index.php.

8 Scheuner MT, Sieverding P, Shekelle PG: Delivery of genomic medicine for common chronic adult diseases: a systematic review. JAMA 2008; 299: 1320-1334.

9 Khoury MJ, Mensah GA: Genomics and the prevention and control of common chronic diseases: emerging priorities for public health action. Prev Chronic Dis 2005; 2: A05.

10 Haga SB, Khoury MJ, Burke W: Genomic profiling to promote a healthy lifestyle: not ready for prime time. Nat Genet 2003; 34: 347-350.

11 Sanderson S, Zimmern R, Kroese M, Higgins J, Patch C, Emery J: How can the evaluation of genetic tests be enhanced? Lessons learned from the ACCE framework and evaluating genetic tests in the United Kingdom. Genet Med 2005; 7: 495-500.

12 Brand AM, Probst-Hensch NM: Biobanking for epidemiological research and public health. Pathobiology 2007; 74: 227-238.

13 Javaher $\mathrm{P}$, Kääriänen $\mathrm{H}$, Kristofferson $\mathrm{U}$ et al: EuroGentest: DNA-based testing for heritable disorders in Europe. Community Genet 2008; 11: 75-120.

14 Godard B, Kaariainen H, Kristoffersson U, Tranebjaerg L, Coviello D, Ayme S: Provision of genetic services in Europe: current practices and issues. Eur J Hum Genet 2003; 11 (Suppl 2): S13-S48.

15 Godard B, ten Kate L, Evers-Kiebooms G, Ayme S: Population genetic screening programmes: principles, techniques, practices, and policies. Eur J Hum Genet 2003; 11 (Suppl 2): S49-S87.

16 European Society of Human Genetics: Provision of genetic services in Europe: current practices and issues. Recommendations of the European Society of Human Genetics. Eur J Hum Genet 2003; 11 (Suppl 2): S2-S4

17 European Society of Human Genetics: Population genetic screening programmes: technical, social and ethical issues. Recommendations of the European Society of Human Genetics. Eur J Hum Genet 2003; 11 (Suppl 2): S5-S7.

18 Ibarreta D, Bock AK, Klein C, Rodriguez-Cerezo E. Towards Quality Assurance and Harmonisation of Genetic Testing Services in the EU. Seville: European Commission Joint Research Centre, 2003.

19 Ibarreta D, Elles R, Cassiman JJ, Rodriguez-Cerezo E, Dequeker E: Towards quality assurance and harmonization of genetic testing services in the European Union. Nat Biotechnol 2004; 22: 1230-1235.

20 Cassiman JJ: Resarch Network: EuroGentest - a European Network of Excellence aimed at harmonizing genetic testing services. Eur J Hum Genet 2005; 13: $1103-1105$

21 Nuffield Council on Bioethics: Genetic Screening: a Supplement to the 1993 Report by the Nuffield Council on Bioethics. London: Nuffield Council on Bioethics 2006.

22 Wilson JMG, Jungner G: Principles and Practice of Screening for Disease. Geneva: World Health Organization, 1968.

23 Kääriäinen $\mathrm{H}$, Hietala $\mathrm{M}$, Kristoffersson $\mathrm{U}$ et al: Recommendations for genetic counselling related to genetic testing. EuroGentest 2009. http://www.eurogentest.org/ web/info/public/unit3/final_recommendations_genetic_counselling.xhtml.

24 Stemerding D, Nelis A: Cancer genetics and its 'different faces of autonomy'. New Genet Soc 2006; 25: 1-19.

25 Calnan M, Wainwright D, Glasner P, Newbury-Ecob R, Ferlie E: Medicine's Next Goldmine? The implications of new genetic health technologies for the health service. Med Health Care Philos 2006; 9: 33-41.

26 Chabas D, Taheri S, Renier C, Mignot E: The genetics of narcolepsy. Annu Rev Genomics Hum Genet 2003; 4: 459-483.

27 Brewerton DA, Hart FD, Nicholls A, Caffrey M, James DCO, Sturrock RD: Ankylosing spondylitis and HL-A 27. Lancet 1973; 1: 904-907.

28 Bomprezzi R, Kovanen PE, Martin R: New approaches to investigating heterogeneity in complex traits. J Med Genet 2003; 40: 553-559.

29 Goldstein DB: Common genetic variation and human traits. N Engl J Med 2009; 360: 1696-1698.

30 Kraft P, Hunter DJ: Genetic risk prediction - are we there yet? N Engl J Med 2009; 360: 1701-1703.

31 The International Schizophrenia Consortium: Common polygenic variation contributes to risk of schizophrenia and bipolar disorder. Nature 2009; 460: 478-752.
32 Guttmacher AE, Porteous ME, McInerney JD: Educating health-care professionals about genetics and genomics. Nat Rev Genet 2007; 8: 151-157.

33 Lewontin RC: The analysis of variance and the analysis of causes. Am J Hum Genet 1974; 26: 400-411.

34 Layzer D: Heritability analyses of IQ scores: science or numerology? Science 1974; 183: 1259-1266.

35 Wallace HM: A model of gene-gene and gene-environment interactions and its implications for targeting environmental interventions by genotype. Theor Biol Med Model 2006; 3: 35

36 Lango $\mathrm{H}$, Weedon $\mathrm{MN}$ : What will whole genome searches for susceptibility genes for common complex disease offer to clinical practice? J Intern Med 2008; 263: 16-27.

37 World Health Organisation: International Classification of Diseases (ICD). http:// www.who.int/classifications/icd/en/.

38 Rose P, Lucassen A: Practical Genetics for Primary Care. Oxford: Oxford University Press, 1999.

39 Ropers HH, Ullmann R: Neue Technologien für Genomforschung und Diagnostik; in Schmidtke J et al. (eds): Gendiagnostik in Deutschland - Status quo und Problemerkun-dung. Limburg an der Lahn, 2007, pp 21-31.

40 Khoury MJ, Beaty TH, Cohen BH: Fundamentals of Genetic Epidemiology. New York: Oxford University Press, 1993.

41 Stewart A, Brice P, Burton H, Pharoah P, Sanderson S, Zimmern R: Genetics, Health Care and Public Policy. An Introduction to Public Health Genetics. Cambridge: Cambridge University Press, 2007.

42 Juul K, Tybjaerg-Hansen A, Schnohr P, Nordestgaard BG: Factor V Leiden and the risk for venous thromboembolism in the adult Danish population. Ann Intern Med 2004; 140: 330-337.

43 Campbell H, Rudan I: Study design in mapping complex disease traits; in Wright AF, Hastie N (eds): Genes and Common Diseases. Cambridge: Cambridge University Press, 2007, pp 92-112.

44 Rimoin DL, Connor JM, Pyeritz RE: Nature and frequency of genetic disease; in Rimoin DL, Connor JM, Pyeritz RE (eds): Emery and Rimoin's Principles and Practice of Medical Genetics, 3rd edn. Edinburgh: Churchill Livingstone, 1997, pp 31-34.

45 Pagon RA: Genetic testing for disease susceptibilities: consequences for genetic counselling. Trends Mol Med 2002; 8: 306-307.

46 International HapMap Consortium: A haplotype map of the human genome. Nature 2005; 437: 1299-1320.

47 van Ommen GJ: Popper revisited. GWAS here last year. Eur J Hum Genet 2008; 16: $1-2$.

48 van Ommen GJ, Cornel MC: Recreational genomics? Dreams and fears on genetic susceptibility screening. Eur J Hum Genet 2008; 16: 403-404.

49 Zeggini E, Scott LJ, Saxena R et al: Meta-analysis of genome-wide association data and large-scale replication identifies additional susceptibility loci for type 2 diabetes. Nat Genet 2008; 40: 638-645.

50 loannidis JPA, Trikalinos TA, Ntzani EE, Contopoulos-loannidis DG: Genetic associations in large versus small studies: an empirical assessment. Lancet 2003; 361: 567-571.

51 Mayo O: The rise and fall of the common disease-common variant (CD-CV) hypothesis: how the sickle cell disease paradigm led us all astray (or did it?). Twin Res Hum Genet 2007; 10: 793-804.

52 Ntzani EE, Rizos EC, loannidis JP: Genetic effects versus bias for candidate polymorphisms in myocardial infarction: case study and overview of large-scale evidence. Am J Epidemiol 2007; 165: 973-984.

53 Keavney B, Palmer A, Parish S et al: International Studies of Infarct Survival (ISIS) Collaborators. Lipid-related genes and myocardial infarction in 4685 cases and 3460 controls: discrepancies between genotype, blood lipid concentrations, and coronary disease risk. Int J Epidemiol 2004; 33: 1002-1013.

54 Liu W, Zhao W, Chase GA: Genome scan meta-analysis for hypertension. Am J Hypertens 2004; 17: 1100-1106.

55 Easton DF, Pooley KA, Dunning AM et al: Genome-wide association study identifies novel breast cancer susceptibility loci. Nature 2007; 447: 1087-1093.

56 Hunter DJ, Kraft P, Jacobs KB et al: A genome-wide association study identifies alleles in FGFR2 associated with risk of sporadic postmenopausal breast cancer. Nat Genet 2007; 39: 870-874.

57 Stacey SN, Monolescu A, Sulem P et al: Common variants on chromosomes 2q35 and $16 q 12$ confer susceptibility to estrogen receptor-positive breast cancer. Nat Genet 2007; 39: 865-869.

58 McPherson R, Pertsemlidis A, Kavaslar N et al: A common allele on chromosome 9 associated with coronary heart disease. Science 2007; 316: 1488-1491.

59 Helgadottir A, Thorleifsson G, Manolescu A et al: A common variant on chromosome 9p21 affects the risk of myocardial infarction. Science 2007; 316: 1491-1493.

60 Frayling TM, Timpson NJ, Weedon MN et al: A common variant in the FTO gene is associated with body mass index and predisposes to childhood and adult obesity. Science 2007; 316: 889-894.

61 Sladek R, Rocheleau G, Rung J et al: A genome-wide association study identifies novel risk loci for type 2 diabetes. Nature 2007; 445: 881-885.

62 Steinthorsdottir V, Thorleifsson G, Reynisdottir I et al: A variant in CDKAL1 influences insulin response and risk of type 2 diabetes. Nat Genet 2007; 39: 770-775.

63 Scott LJ, Mohlke KL, Bonnycastle LL et al: A genome-wide association study of type 2 diabetes in Finns detects multiple susceptibility variants. Science 2007; 316: $1341-1345$. 
64 Zeggini E, Weedon MN, Lindgren $\mathrm{CM}$ et al: Replication of genome-wide association signals in UK samples reveals risk loci of type 2 diabetes. Science 2007; 316: 1336-1341.

65 Yeager M, Orr N, Hayes RB et al: Genome-wide association study of prostate cancer identifies a second risk locus at 8q24. Nat Genet 2007; 39: 645-649.

66 Gudmundsson J, Sulem P, Manolescu A et al: Genome-wide association study identifies a second prostate cancer susceptibility variant at 8q24. Nat Genet 2007; 39: 631-637.

67 Khoury MJ, Wacholder S: Invited commentary: from genome-wide association studies to gene-environment-wide interaction studies: challenges and opportunities. $\mathrm{Am} \mathrm{J}$ Epidemiol 2009; 169: 227-230.

68 Wray NR, Goddard ME, Visscher PM: Prediction of individual risk of complex disease. Curr Opin Genet Dev 2008; 18: 257-263.

69 Clayton DG: Prediction and interaction in complex disease genetics: experience in type 1 diabetes. PLoS Genet 2009; 5: e1000540.

70 Janssens ACJW, van Duijn CM: Genome-based prediction of common diseases: advances and prospects. Hum Mol Genet 2008; 17: R166-R173.

71 Gartner CE, Barendregt JJ, Hall WD: Multiple genetic tests for susceptibility to smoking do not outperform simple family history. Addiction 2009; 104: 118-126.

72 Munafò MR: The clinical utility of genetic tests. Addiction 2009; 104: 127-128.

73 Jakobsdottir J, Gorin MB, Conley YP, Ferrell RE, Weeks DE: Interpretation of genetic association studies: markers with replicated highly significant odds ratios may be poor classifiers. PLoS Genet 2009; 5: e1000337.

74 Baker SG, Kaprio J: Common susceptibility genes for cancer: search for the end of the rainbow. BMJ 2006; 332: 1150-1152.

75 Antoniou AC, Pharoah PDP, McMullan G et al: A comprehensive model for familial breast cancer incorporating BRCA1, BRCA2 and other genes. Br J Cancer 2002; 86: 76-83.

76 Khoury MJ, Burke W, Thomson E: Genetics and Public Health in the 21st Century. New York: Oxford University Press, 2000.

77 Vineis P, Schulte P, McMichael AJ: Misconceptions about the use of genetic tests in populations. Lancet 2001; 357: 709-712.

78 Khoury MJ, Wagener DK: Epidemiological evaluation of the use of genetics to improve the predictive value of disease risk factors. Am J Hum Genet 1995; 56: 835-844.

79 Khoury MJ, Yang Q, Gwinn M, Little J, Flanders WD: An epidemiologic assessment of genomic profiling for measuring susceptibility to common diseases and targeting interventions. Genet Med 2004; 6: 38-47.

80 Goel V: Appraising organised screening programmes for testing for genetic susceptibility to cancer. BMJ 2001; 322: 1174-1178.

81 Burke W, Coughlin SS, Lee NC, Weed DL, Khoury MJ: Application of population screening principles to genetic screening for adult-onset conditions. Genet Test 2001; 5: 201-211.

82 Khoury MJ, McCabe LL, McCabe ERB: Population screening in the age of genomic medicine. N Engl J Med 2003; 348: 50-58.

83 CDC: ACCE model system for collecting, analyzing and disseminating information on genetic tests. http://www.cdc.gov/genomics/gtesting/ACCE/FBR/index.htm.

84 Haddow JE, Palomaki GE: ACCE: a model process for evaluating data on emerging genetic tests; in Khoury MJ, Little J, Burke W (eds): Human Genome Epidemiology. New York: Oxford University Press, 2004, pp 217-233.

85 EGAPP: Evaluation of genomic applications in practice and prevention. http:// www.egappreviews.org/default.htm.

86 Kroese M, Zimmern RL, Farndon P, Stewart F, Whittaker J: How can genetic tests be evaluated for clinical use? Experience of the UK Genetic Testing Network. Eur J Hum Genet 2007; 15: 917-921.

87 UK Genetic Testing Network: Gene dossiers. http://www.ukgtn.nhs.uk/gtn/Information/ Services/Gene+Dossiers

88 CanGèneTest. http://www.cangenetest.org.

$89 \mathrm{GfH}$ : Deutsche Gesellschaft für Humangenetik e.v. http://ww.gfhev.de.

90 Eurogentest: Clinical Utility Gene Cards. http://www.eurogentest.org/web/info/public/ unit3/geneCards.xhtml.

91 Wald NJ, Hackshaw AK, Frost CD: When can a risk factor be used as a worthwhile screening test? BMJ 1999; 319: 1562-1565.

92 CDC: ACCE: a CDC-sponsored project (2000-2004). http://www.cdc.gov/genomics/ gtesting/ACCE/acce_proj.htm\#T1.

93 Burke W, Zimmern RL: Moving Beyond ACCE - An Expanded Framework for Genetic Test Evaluation. Cambridge: PHG Foundation, 2007.

94 Angastiniotis M, Modell B: Global epidemiology of hemoglobin disorders. Ann N Y Acad Sci 1998; 850: 251-269.

95 Lyssenko V, Almgren P, Anevski D. et al: Genetic prediction of future type 2 diabetes. PLoS Med 2005; 2: e345.

96 Janssens ACJW, Gwinn M, Subramonia-lyer S, Khoury MJ: Does genetic testing really improve the prediction of type 2 diabetes? PLoS Med 2006; 3: e114.

97 Janssens ACJW, Gwinn M, Valdez R, Venkat Narayan KM, Khoury MJ: Predictive genetic testing of type 2 diabetes. BMJ 2006; 333: 509-510.

98 Janssens ACJW, Khoury MJ: Predictive value of testing for multiple genetic variants in multifactorial diseases: implications for the discourse on ethical, legal and social issues. IJPH 2006; 3: 35-41.

99 Ridker PM, Cook NR: Biomarkers for prediction of cardiovascular events. N Engl J Med 2007; 356: 1472-1473; author reply 1474-1475.

100 Bartlett JMS: Pharmacodiagnostic testing in breast cancer: focus on HER2 and trastuzumab therapy. Am J Pharmacogenomics 2005; 5: 303-315.
101 Glas AM, Floore A, Delahaye $L$ et al: Converting a breast cancer microarray signature into a high-throughput diagnostic test. BMC Genomics 2006; 7: 278.

102 Mook S, Van't Veer LJ, Rutgers EJ, Piccart-Gebhart MJ, Cardoso F: Individualization of therapy using Mammaprint: from development to the MINDACT Trial. Cancer Genomics Proteomics 2007; 4: 147-155.

103 Gail MH: Discriminatory accuracy from single-nucleotide polymorphisms in models to predict breast cancer risk. JNCI 2008; 100: 1037-1041.

104 Pharoah PDP, Antoniou AC, Easton DF, Ponder BAJ: Polygenes, risk prediction, and targeted prevention of breast cancer. N Engl J Med 2008; 358 : 2796-2803.

105 Ripperger T, Gadzicki D, Meindl A, Schlegelberger B: Breast cancer susceptibility: current knowledge and implications for genetic counselling. Eur J Hum Genet 2009; 17: $722-731$.

106 Lubinski J, Korzen M, Gorski B et al: Breast cancer susceptibility genes. J BUON 2007; 12: S23-S29.

107 Rowley PT, Haddow JE, Palomaki GE: DNA testing strategies aimed at reducing morbidity and mortality from hereditary non/polyposis colorectal cancer (HNPCC). An ACCE mini review. http://www.cdc.gov/genomics/gtesting/file/print/FBR/CC_ Introduction.pdf.

108 Damani SB, Topol EJ: Future use of genomics in coronary artery disease. JACC 2007; 50: $1933-1940$

109 Tan HL, Hofman N, van Langen IM, van der Wal AC, Wilde AAM: Sudden unexplained death: heritability and diagnostic yield of cardiological and genetic examination in surviving relatives. Circulation 2005; 112: 207-213.

110 Dekker LRC, Bezzina CR, Henriques JPS. et al: Familial sudden death is an important risk factor for primary ventricular fibrillation: a case-control study in acute myocardial infarction patients. Circulation 2006; 114: 1140-1145.

111 Vaxillaire M, Froguel P: Monogenic diabetes in the young, pharmacogenetics and relevance to multifactorial forms of Type 2 diabetes. Endocr Rev 2008; 29: 254-264.

112 Stolerman ES, Florez JC: Genomics of type 2 diabetes mellitus: implications for the clinician. Nat Rev Endocrinol 2009; 5: 429-436.

113 Mearin ML, Ivarsson A, Dickey W: Coeliac disease: is it time for mass screening? Best Pract Res Clin Gastroenterol 2005; 19: 441-452.

114 Cacabelos R: Pharmacogenomics in Alzheimer's disease. Methods Mol Biol 2008; 448: 213-357.

115 NHS National Institute for Health and Clinical Excellence. http://www.nice.org.uk/ TA111.

116 Vandenbroucke JP, van der Meer FJ, Helmerhorst FM, Roosendaal FR: Factor V Leiden: should we screen oral contraceptive users and pregnant women? BMJ 1996; 313: 1127-1130.

117 Cohn DM, Roshani S, Middeldorp S: Thrombophilia and venous thromboembolism: implications for testing. Semin Thromb Hemost 2007; 33: 573-581.

118 Agency for Healthcare Research and Quality: Outcomes of genetic testing in adults with a history of venous thromboembolism. http://www.ahrq.gov/Clinic/tp/fvltp.htm.

119 Segal JB, Brotman DJ, Necochea AJ. et al: Predictive value of Factor V Leiden and Prothrombin G20210A in adults with venous thromboembolism and in family members of those with a mutation. A systematic review. JAMA 2009; 301: 2472-2485.

120 Newson AJ, Humphries SE: Cascade testing in familial hypercholesterolaemia: how should family members be contacted? Eur J Hum Genet 2005; 13: 401-408.

121 Yoon PW, Scheuner MT, Khoury MJ: Research priorities for evaluating familyhistory in the prevention of common chronic diseases. Am J Prev Med 2003; 24: 128-135.

122 Umans-Eckenhausen M, Defesche J, Sijbrands E, Scheerder R, Kastelein J: Review of first 5 years of screening for familial hypercholesterolaemia in the Netherlands. Lancet 2001; 357: 165-168.

123 Burke W, Cogswell M, McDonnell S: Public health strategies to prevent the complications of hemochromatosis; in Khoury MJ, Burke W, Thomson EJ (eds): Oxford Monographs on Medical Genetics. Genetics and Public Health in the 21st Century: Using Genetic Information to Improve Health and Prevent Disease. New York: Oxford University Press, 2000, pp 447-462.

124 Bradley LA, Johnson DD, Palomaki GE, Haddow JE, Robertson NH, Ferrie RM: Hereditary haemochromatosis mutation frequencies in the general population. J Med Screen 1998; 5: 34-36.

125 Adams P, Brissot P, Powell LW: EASL International Consensus Conference on Haemochromatosis. J Hepatol 2000; 33: 485-504.

126 Burke W, Thomson E, Khoury MJ et al: Hereditary hemochromatosis: gene discovery and its implications for population-based screening. JAMA 1998; 280: 172-178.

127 Hanson EH, Imperatore G, Burke W: HFE gene and hereditary hemochromatosis: a HuGE review. Am J Epidemiol 2001; 154: 193-206.

128 Beutler E, Felitti VJ, Koziol JA, HO NJ, Gelbart T: Penetrance of 845G $\rightarrow$ A (C282Y) HFEhereditary haemochromatosis mutation in the USA. Lancet 2002; 359: 211-218.

129 Allen KJ, Gurrin LC, Constantine CC et al: Iron-overload-related disease in HFE hereditary hemochromatosis. N Engl J Med 2008; 358: 221-230.

130 Stuhrmann M, Strassburg C, Schmidtke J: Genotype-based screening for hereditary haemochromatosis. I: Technical performance, costs and clinical relevance of a German pilot study. Eur J Hum Genet 2005; 13: 69-78.

131 Stuhrmann M, Hoy L, Nippert I, Schmidtke J: Genotype-based screening for hereditary haemochromatosis. II: Attitudes towards genetic testing and psychosocial impact - a report from a German pilot study. Genet Test 2005; 9: 242-254. 
132 Bulaj ZJ, Ajioka RS, Phillips JD et al: Disease-related conditions in relatives of patients with hemochromatosis. N Engl J Med 2000; 343: 1529-1535.

133 El-Serag HB, Inadomi JM, Kowdley KV: Screening for hereditary hemochromatosis in siblings and children of affected patients. A cost-effectiveness analysis. Ann Intern Med 2000; 132: 261-269.

134 McCune CA, Ravine D, Worwood M, Jackson HA, Evans HM, Hutton D: Screening for hereditary haemochromatosis within families and beyond. Lancet 2003; 362: 1897-1898.

135 Powell LW, Dixon JL, Hewett DG: Role of early case detection by screening relatives of patients with HFE-associated hereditary haemochromatosis. Best Pract Res Clin Haematol 2005; 18: 221-234.

136 McCune CA, Ravine D, Carter $\mathrm{K}$ et al: Iron loading and morbidity among relatives of HFE C282Y homozygotes identified either by population genetic testing or presenting as patients. Gut 2006; 55: 554-562.

137 Powell LW, Dixon JL, Ramm GA et al: Screening for hemochromatosis in asymptomatic subjects with or without a family history. Arch Intern Med 2006; 166: 294-301.

138 Jacobs EM, Hendriks JC, Marx JJ et al: Morbidity and mortality in first-degree relatives of $\mathrm{C} 282 \mathrm{Y}$ homozygous probands with clinically detected haemochromatosis compared with the general population: the HEmochromatosis FAmily Study (HEFAS). Neth J Med 2007; 65: 425-433.

139 Health Council of the Netherlands: Screening for Haemochromatosis. The Hague: Health Council of the Netherlands, 1999. Publication no. 1999/21. ISBN 90-5549-288-4

140 Ombiga J, Adams LA, Tang K, Trinder D, Olynyk JK: Screening for HFE and iron overload. Semin Liver Dis 2005; 25: 402-410.

141 Jacobs EM, Meulendijks CF, Elving L, van der Wilt GJ, Swinkels DW: Impact of the introduction of a guideline on the targeted detection of hereditary haemochromatosis. Neth J Med 2005; 63: 205-214.

142 Pietrangelo A: Hereditary hemochromatosis - a new look at an old disease. $N$ Engl J Med 2004; 350: 2383-2397.

143 Njajou OT, Alizadeh BZ, van Duijn CM: Is genetic screening for hemochromatosis worth while? Eur J Epidemiol 2004; 19: 101-108.

144 Bryant J, Cooper K, Picot J et al: A systematic review of the clinical validity and clinical utility of DNA testing for hereditary haemochromatosis type 1 in at-risk populations. J Med Genet 2008; 45: 513-518.

145 Rogowski WH: The cost-effectiveness of screening for hereditary hemochromatosis in Germany: a remodeling study. Med Decis Making 2009; 29: 224-238.

146 Jacobs EM: On the Early Management of Hereditary Haemochromatosis. Nijmegen: Dissertation Radboud Universiteit Nijmegen, 2007. http://webdoc.ubn.ru.nl/mono/j/ jacobs_e/on_theam.pdf.

147 Baars MJ, Henneman L, Ten Kate LP: Deficiency of knowledge of genetics and genetic tests among general practitioners, gynecologists, and pediatricians: a global problem. Genet Med 2005; 7: 605-610.

148 Martin G, Currie G, Finn R: Bringing genetics into primary care: findings from a national evaluation of pilots in England. J Health Serv Res Policy 2009; 14: 204-211.

149 Teutsch SM, Bradley LA, Palomaki GE et al: The Evaluation of Genomic Applications in Practice and Prevention (EGAPP) initiative: methods of the EGAPP Working Group. Genet Med 2009; 11: 3-14.

150 Siegel JE, Weinstein MC, Russell LB, Gold MR: Recommendations for reporting costeffectiveness analyses. Panel on Cost-Effectiveness in Health and Medicine. JAMA 1996; 276: 1339-1341.

151 Drummond MF, Jefferson TO: Guidelines for authors and peer reviewers of economic submissions to the BMJ. The BMJ Economic Evaluation Working Party. BMJ 1996; 313: 275-283

152 Briggs A, Claxton K, Sculpher M: Decision Modelling for Health Economic Evaluation. Oxford: Oxford University Press, 2006.

153 Rogowski W: Genetic screening by DNA technology: a systematic review of health economic evidence. Int J Technol Assess Health Care 2006; 22 327-237.

154 Drummond MF, Sculpher MJ, Torrance GW, O'Brien BJ, Stoddart GL: Methods for the Economic Evaluation of Health Care Programmes, 3rd edn. Oxford/New York: Oxford University Press, 2006.

155 Rogowski W, Langer A: Gentests und deren Einsatz für Reihenuntersuchungen aus gesundheitsökonomischer Perspektive; in Schmidtke J, Müller-Röber B, van den Daele W et al (eds): Gendiagnostik in Deutschland: Status quo und Problemerkundung. Limburg an Lahn, 2007, pp 87-106.

156 Prévost $\mathrm{C}$, Veillette $\mathrm{S}$, Perron $\mathrm{M}$ et al: Psychosocial impact of predictive testing for myotonic dystrophy type 1 . Am J Med Genet 2004; 126: 68-77.

157 Veenstra DL: The interface between epidemiology and pharmacogenomics; in Khoury MJ, Little J, Burke W (eds): Human Genome Epidemiology. New York: Oxford University Press, 2004, pp 234-246.

158 Grosse SD, Khoury MJ: What is the clinical utility of genetic testing? Genet Med 2006; 8: 448-450.

159 Khoury MJ, Jones K, Grosse SD: Quantifying the health benefits of genetic tests: The importance of a population perspective. Genet Med 2006; 8: 191-195.

160 Grosse SD, Wordsworth S, Payne K et al: Economic methods for valuing the outcomes of genetic testing: beyond cost-effectiveness analysis. Genet Med 2008; 10: 648-654.

161 McGuire AL, Burke W: An unwelcome side effect of direct-to-consume personal genome testing: raiding the medical commons. JAMA 2008; 300 : 2669-2671.
162 Schöffski O: Genetic testing and the health care system. Eur J Health Econom 2002; 3: 73-76.

163 Carlson JJ, Henrikson NB, Veenstra DL, Ramsey SD: Economic analyses of human genetics services: a systematic review. Genet Med 2005; 7: 519-523.

164 Philips Z, Ginnelly L, Sculpher M et al: Review of guidelines for good practice in decision-analytic modelling in health technology assessment. Health Technol Assess 2004; 8: 1-158.

165 Evers S, Goossens M, de Vet H, van Tulder M, Ament A: Criteria list for assessment of methodological quality of economic evaluations: Consensus on Health Economic Criteria. Int J Technol Assess Health Care 2005; 21: 240-245.

166 Culyer A, McCabe C, Briggs A et al: Searching for a threshold, not setting one: the role of the National Institute for Health and Clinical Excellence. J Health Serv Res Policy 2007; 12: 56-58.

167 Arrow KJ: Uncertainty and the welfare economics of medical care. J Health Polit Policy Law 2001; 26: 851-883.

168 Giacomini M, Miller F, O'Brien BJ: Economic considerations for health insurance coverage of emerging genetic tests. Community Genet 2003; 6: 61-73.

169 Griffith GL, Edwards RT, Gray J: Cancer genetics services: a systematic review of the economic evidence and issues. Br J Cancer 2004; 90: 1697-1703.

170 Jarrett J, Mugford M: Genetic health technology and economic evaluation: a critical review. Appl Health Econ Health Policy 2006; 5: 27-35.

171 Rogowski W: Overview: health economic evaluations of pharmacogenetics \& individualized medicine. Poster presentation at the ESF-IfW Research Conference 'New Medical Technology and Medical Decision Making', Salzau Castle, 2006.

172 Sassi F, Archard L, McDaid D: Searching literature databases for health care economic evaluations: how systematic can we afford to be? Med Care 2002; 40: 387-394.

173 Rogowski W, Grosse SD, Khoury MJ: Challenges of translating genetic tests into clinical and public health practice. Nat Rev Genet 2009; 10: 489-495.

174 Marska N: Cost effectiveness analysis of a prophylactic, genetic-oncological programme in the West Pomeranian Region - initial announcement of results. Hered Cancer Clin Pract 2006; 4: 69.

175 Devlin N, Parkin D: Does NICE have a cost-effectiveness threshold and what other factors influence its decisions? A binary choice analysis. Health Econ 2004; 13: 437-452.

176 Ropers $\mathrm{HH}$ : New perspectives for the elucidation of genetic disorders. Am J Hum Genet 2007; 81: 199-207.

177 loannidis J, Ntzani E, Trikalinos T, Contopoulos-loannidis D: Replication validity of genetic association studies. Nat Genet 2001; 29: 306-309.

178 Bell J: The new genetics in clinical practice. BMJ 1998; 316: 618-620.

179 Holtzman NA, Marteau TM: Will genetics revolutionize medicine? N Engl J Med 2000; 343: 141-144.

180 Department of Health: Our Inheritance, Our Future. Realising the Potential of Genetics in the NHS. London: the Stationery Office, 2003. http://www.dh. gov.uk/prod_consum_dh/groups/dh_digitalassets/@dh/@en/documents/digitalasset/dh_ 4019239.pdf.

181 Calnan M, Wainwright D, Glasner P, Newbury-Ecob R, Ferlie E: Medicine's next goldmine? The implications of new genetic health technologies for the health service. Medicine Health Care Philos 2006; 9: 39.

182 Evans JP: Health care in the age of genetic medicine. JAMA 2007; 298: 2670-2672.

183 Farmer Y, Godard B: From scientific considerations to ethical integration. Genomics Soc Policy 2007; 3: 14-27.

184 House of Lords Science and Technology Committee: Genomic Medicine. London: House of Lords Science and Technology Committee, 2009. http://www. publications. parliament.uk/pa/ld200809/Idselect/ldsctech/107/107i.pdf.

185 Lucassen A, Watson E, Harcourt J, Rose P, O'Grady J: Guidelines for referral to regional genetics service: GPs respond by referring more appropriate cases. Fam Pract 2001; 18: 135-140.

186 World Health Report 2002: Reducing Risks, Promoting Healthy Life. Geneva: World Health Organization, 2002.

187 Hunter DJ, Khoury MJ, Drazen JM: Letting the genome out of the bottle- will we get our wish? N Engl J Med 2008; 358: 105-107.

188 Patch C, Sequeiros J, Cornel MC: Genetic horoscopes: is it all in the genes? Points for regulatory control of direct-to-consumer genetic testing. Eur J Hum Genet 2009; 17 : 857-859.

189 Wrick KL: Direct-to-consumer genetic testing: business prospects in the United States, Insight Pharma Rep 2009.

190 Human Genetics Commission: More Genes Direct. A Report on Developments in the Availability, Marketing and Regulation of Genetic Tests Supplied Directly to the Public. London: HGC, 2007. http://www.hgc.gov.uk/UploadDocs/DocPub/Document/ More\%20Genes\%20Direct.pdf.

191 Hogarth S, Melzer D: The IVD Directive and Genetic Testing. Problems and Proposals. A Briefing Presented to the 20th Meeting of Competent Authorities. Lisbon, 2007. http://ec.europa.eu/enterprise/sectors/medical-devices/files/recast_docs_2008/ responses/132-r-2_en.pdf.

192 Hogarth S, Javitt G, Melzer D: The current landscape for direct-to-consumer genetic testing: legal, ethical and policy issues. Annu Rev Genomics Hum Genet 2008; 9: 161-182.

193 Mihaescu R, van Hoek M, Sijbrands EJG. et al: Evaluation of risk prediction updates from commercial genome-wide scans. Genet Med 2009; 11: 588-594.

194 Offit K: Genomic profiles for disease risk. Predictive or premature? JAMA 2008; 299. $1353-1355$ 
195 Allison M: Regulation of consumer genomic tests remains in limbo. Nat Biotechnol 2009; 27: 875-877.

196 Khoury MJ, Berg A, Coates R, Evans J, Teutsch SM, Bradley LA: The evidence dilemma in genomic medicine. Health Affairs 2008; 27: 1600-1611.

197 Melzer D, Hogarth S, Liddell K, Ling T, Sanderson S, Zimmern R: Genetic tests for common diseases: new insights, old concerns. BMJ 2008; 336: 590-593.

198 Lunshof JE, Chadwick R, Church GM: Hippocrates revisited? Old ideals and new realities. Genomic Med 2008; 2: 1-3.

199 Harding A: Do it yourself cancer gene testing raises concerns. BMJ 2005; 330: 617 .

200 Kolor K, Liu T, St Pierre J, Khoury MJ: Health care provider and consumer awareness, perceptions, and use of direct-to-consumer personal genomic tests, United States, 2008. Letter to the Editor. Genet Med 2009; 11: 595.

201 McGuire A, Diaz CM, Wang T, Hilsenbeck S: Social networkers'attitudes toward direct-to-consumer personal genome testing. Am J Bioethics 2009; 9: 3-10.

202 Howard H, Borry P: Direct-to-consumer genetic testing: more questions than benefits? Personalized Med 2008; 5: 317-320.

203 Howard HC, Borry P: Personal genome testing: do you know what you are buying? Am J Bioethics 2009; 9: 11-13.

204 Borry P, Howard HC, Sénécal K, Avard D: Direct-to-consumer genome scanning services. Also for children? Nat Rev Genet 2009; 10: 666-667.

205 Human Genetics Commission: Genes Direct. Ensuring the Effective Oversight of Genetic Tests Supplied Directly to the Public. London: HGC, 2003.

206 Hudson K, Javitt G, Burke W, Byers P, ASHG Social Issues Committee: ASHG statement on direct-to-consumer genetic testing in the United States. Am J Hum Genet 2007; 81: 635-637.

207 Ng PC, Murray SS, Levy S, Venter JG: An agenda for personalized medicine. Nature 2009; 461: 724-726.

208 Gurwitz D, Bregman-Eschet Y: Personal genomics services: whose genomes? Eur J Hum Genet 2009; 17: 883-889.

209 Human Genetics Commission: HGC publishes consultation on direct-to-consumer genetic testing. http://www.hgc.gov.uk/Client/news_item.asp? Newsid=132.

210 Health Council of the Netherlands: Screening: Between Hope and Hype. The Hague: Health Council of the Netherlands, 2008. Publication no. 2008/05E. http://www. gezondheidsraad.nl/en/publications/screening-between-hope-and-hype-0\#a-downloads.

211 The European Nutrigenomics Organisation: What is nutrigenomics? http://www. nugo.org/everyone/24023.

212 Wallace $\mathrm{H}$ : Your diet tailored to your genes. Preventing diseases or misleading marketing? A report by Genewatch UK, 2006. http://www.genewatch.org/uploads/ f03c6d66a9b354535738483c1c3d49e4/Nutrigenomics.pdf.

213 Khoury MJ, Gwinn M, Yoon PW, Dowling N, Moore CA, Bradley L: The continuum of translation research in genomic medicine: how can we accelerate the appropriate integration of human genome discoveries into health care and disease prevention? Genet Med 2007; 9: 665-674.

214 Achterbergh R, Lakeman P, Stemerding D, Moors EHM, Cornel MC: Implementation of preconceptional carrier screening for cystic fibrosis and haemoglobinopathies: a sociotechnical analysis. Health Policy 2007; 83: 277-286.

215 Stemerding D, van Berkel D: Maternal serum screening, political decision-making and social learning. Health Policy 2001; 56: 111-125.

216 Moors EHM, Rip A, Wiskerke JSC: The dynamics of innovation: a multilevel coevolutionary perspective; in Wiskerke JSC, van der Ploeg JD (eds): Seeds of Transition. Essays on Novelty Production, Niches and Regimes in Agriculture. Assen: Koninklijke Van Gorcum, 2004, pp 31-56.

217 Stemerding D, Swierstra T: How might scenario studies help us to think about the normative implications of genomics and predictive medicine?; in de Bouvet A, Boitte P, Aiguier G (eds): Questions éthiques en médecine predictive. Paris Montrouge: John Libbey Eurotext, 2006, pp 81-88.

218 Davison C, Macintyre S, Davey Smith G: The potential social impact of predictive genetic testing for susceptibility to common chronic diseases: a review and proposed research agenda. Sociol Health III 1994; 16: 340-370.

219 Stemerding D, Swierstra T, Boenink M: Exploring the dynamic mutual interaction of technology and morality in the field of genetic susceptibility testing: a scenario study. Futures 2010; 42: 1133-1145.

220 Foster MW, Mulvihill JJ, Sharp RR: Evaluating the utility of personal genomic information. Genet Med 2009; 11: 570-574.

221 Van Rijswoud E, Stemerding D, Swierstra TE: Genetica, genomics en gezondheidszorg. Een toekomstverkenning. Nijmegen: Centre for Society and Genomics, 2008. http://www.society-genomics.nl/uploads/media/Genetica-genomics-gezondheidszorg. pdf.

222 Janssens ACJW, Khoury MJ: Predictive value of testing for multiple genetic variants in multifactorial diseases: implications for the discourse on ethical, legal and social issues. IJPH 2006; 3: 35

223 Marteau TM, van Duijn M, Ellis I: Effects of genetic screening on perceptions of health: a pilot study. J Med Genet 1992; 29: 24-26.

224 Denayer L, Welkenhuysen M, Evers-Kiebooms G, Cassiman JJ, van den Berghe H: The $\mathrm{CF}$ carrier status is not associated with a diminished self-concept or increased anxiety: results of psychometric testing after at least 1 year. Clin Genet 1996; 49: 232-236.

225 Michie S, Smith JA, Senior V, Marteau TM: Understanding why negative genetic test results sometimes fail to reassure. Am J Med Genet 2003; 119: 340-347.

226 Marteau TM, Lerman C: Genetic risk and behavioural change. BMJ 2001; 322 : 1056-1059.

227 Tibben A: Predictive testing for Huntington's disease. Brain Res Bull 2007; 72: 165-171.
228 Saukko PM, Ellard S, Richards SH, Shepherd MH, Campbell JL: Patient's understanding of genetic susceptibility testing in mainstream medicine: qualitative study on thrombophilia. BMC Health Serv Res 2007; 7: 82

229 Pijl M, Timmermans DRM, Claassen L et al: Impact of communicating familial risk of diabetes on illness perceptions and self-reported behavioral outcomes: a randomized controlled trial. Diabetes Care 2009; 32: 597-599.

230 Gason AA, Aitken MA, Metcalfe SA, Allen KJ, Delatycki MB: Genetic susceptibility screening in schools: attitudes of the school community towards hereditary haemochromatosis. Clin Genet 2004; 67: 166-174.

231 Power TE, Adams PC, Barton JC et al: Psychosocial impact of genetic testing for hemochromatosis in the HEIRS Study: a comparison of participants recruited in Canada and in the United States. Genet Test 2007; 11: 55-64.

232 Picot J, Bryant J, Cooper K et al: Psychosocial aspects of DNA testing for hereditary hemochromatosis in at-risk individuals: a systematic review. Genet Test Mol Biomarkers 2009; 13: 7-14

233 Senior V, Marteau TM, Peters TJ: Will genetic testing for predisposition for disease result in fatalism? A qualitative study of parents responses to neonatal screening for familial hypercholesterolaemia. Soc Sci Med 1999; 48: $1857-1860$

234 Van Maarle MC, Stouthard ME, Bonsel GJ: Quality of life in a family based genetic cascade screening programme for familial hypercholesterolaemia: a longitudinal study among participants. J Med Genet 2003; 40: e3.

235 Claassen L, Henneman L, Kindt I, Marteau TM, Timmermans DRM: Perceived risk and representations of cardiovascular disease and preventive behavior in people diagnosed with familial hypercholesterolemia: a cross-sectional questionnaire study. J Health Psychol 2010; 15: 33-43.

236 Marteau TM, Weinman J: Self-regulation and the behavioural response to DNA risk information: a theoretical analysis and framework for future research. Soc Sci Med 2006; 62: 1360-1368.

237 Arribas-Ayllon M, Bartlett A, Featherstone K: Complexity and accountability: the witches' brew of psychiatric genetics. Soc Stud Sci 2010; 40: 499-524.

238 Beeri MS, Rapp M, Silverman JM et al: Coronary artery disease is associated with Alzheimer disease neuropathology in APOE4 carriers. Neurology 2006; 66 : 1399-1404.

239 Corbellini G: Genetic risk, medical education, public understanding of genetics, and evolutionary medicine: the challenges of genetic counselling for complex disorders. Topoi 2004; 23: 187-193.

240 Council of Europe: Additional Protocol to the Convention on Human Rights and Biomedicine, Concerning Genetic Testing for Health Purposes. Strasbourg: Council of Europe, 2008. http://conventions.coe.int/treaty/EN/Treaties/Html/ 203.htm.

241 Lwoff L: Council of Europe adopts protocol on genetic testing for health purposes. Eur J Hum Genet 2009; 17: 1374-1377.

242 Shepperd S: DISCERN-Genetics: quality criteria for information on genetic testing. Eur J Hum Genet 2006; 14: 1179-1188.

243 Discern genetics: Welcome to DISCERN genetics. http://www.discern-genetics.org/.

244 Marang-van de Mheen PJ, van Maarle MC, Stouthard MEA: Getting insurance after genetic screening on familial hypercholesterolaemia; the need to educate both insurers and the public to increase adherence to national guidelines in the Netherlands. J Epidemiol Community Health 2002; 56: 145-147.

245 Homsma SJ, Huijgen R, Middeldorp S, Sijbrands EJ, Kastelein JJ: Molecular screening for familial hypercholesterolaemia: consequences for life and disability insurance. Eur J Hum Genet 2008; 16: 14-17.

246 Association of British Insurers: insurance genetics moratorium extended to 2014 http://www.abi.org.uk/Media/Releases/2008/06/Insurance_genetics_moratorium_ extended_to_2014.aspx.

247 Soini S, Aymé S, Matthijs G: Patenting and licensing in genetic testing: ethical, legal and social issues. Eur J Hum Genet 2008; 16: S10-S50.

248 Aymé S, Matthijs G, Soini S: Patenting and licensing in genetic testing. Eur J Hum Genet 2008; 16: S3-S9.

249 Wynne B: Misunderstood misunderstanding: social identities and public uptake of science. Public Underst Sci 1992; 1: 281-304.

250 Weldon S: Public Engagement in Genetics: A Review of Current Practice in the UK. A Report for NOWGEN. Lancaster: Lancaster University, 2004

251 Toumey C: Science and democracy. Nat Nanotechnol 2006; 1: 6-7.

252 Calnan M, Wainwright D, Glasner P, Newbury-Ecob R, Ferlie E: Medicine's next goldmine? The implications of new genetic health technologies for the health service. Med Health Care Philos 2006; 9: 36.

253 Centre for Society and Genomics. http://www.society-genomics.nl.

254 Dougherty MJ: Closing the gap: inverting the genetics curriculum to ensure an informed public. Am J Hum Genet 2009; 85: 6-12.

255 Coviello DA, Skirton H, Cerrato N Lewis C, Kent A: Genetic testing and counselling in Europe: health professionals current educational provision, needs assessment and potential strategies for the future. Eur J Hum Genet 2007; 15: 1203-1204.

256 Eurogentest: Unit Six Database of health professional organisations in EU member states with relevance to genetics. http://www.eurogentest.org/web/info/public/unit6/ bodies.xhtml.

257 Capability Project. http://www.capabilitynet.eu.

258 Lell B, Ruangweerayut R, Wiesner J et al: Fosmidomycin, a novel chemotherapeutic agent for malaria. Antimicrob Agents Chemother 2003; 47: 735-738.

259 Christianson A, Modell B: Medical genetics in developing countries. Annu Rev Genomics Hum Genet 2004; 5: 219-265. 
260 Directive 98/79/EC of the European Parliament and of the Council of 27 October 1998 on in vitro diagnostic medical devices. http://eur-lex.europa.eu/LexUriServ/ LexUriserv.do?uri=CELEX:31998L0079:EN:HTML.

261 Study Group 1 of the Global Harmonization Task Force: Principles of In Vitro Diagnostic (IVD) Medical Devices Classification, Proposed Document. GHTF, 2007. http://www.ghtf.org/documents/sg1/SG1-PD-N45-R12.pdf.

262 MEDDEV: 2.14/2 rev.1, February 2004 IVD Guidance: Research Use Only products. A guide for manufacturers and notified bodies. http://ec.europa.eu/enterprise/sectors/ medical-devices/files/meddev/2_14_2_research_only_product_en.pdf.

263 Higson GR: Medical Device Safety - The Regulation of Medical Devices for Public Health and Safety. Bristol: Institute of Physics, 2002.

264 Commission decision of 7 May 2002 on common technical specifications for in vitrodiagnostic medical devices. http://eur-lex.europa.eu/LexUriServ/LexUriServ.do?ur$\mathrm{i}=$ OJ:L:2002:131:0017:0030:EN:PDF.

265 Hausman ED, Altaie SS: Regulatory aspects of total product lifecycle. Diabetes Technol Therap 2004; 6: 761-766.

266 Park R: An office of its own (Interview with S Gutman). IVD Technol 2003; 9. http:// www.ivdtechnology.com/article/office-its-own.

267 European Association of Notified Bodies for Medical Devices. NB-MED/2.12/Rec1: Post-marketing Surveillance (PMS) post-market/production.

268 European Commission: Guidelines on post market clinical follow-up MEDDEV 2.12 2, 2004

269 Holtzman NA, Watson MS: Promoting Safe and Effective Genetic Testing in the United States. Final Report of the Task Force on Genetic Testing. Baltimore: Johns Hopkins University Press, 1998.

270 FDA: Office of In Vitro Diagnostic Device Evaluation and Safety. http://www.fda.gov/ AboutFDA/CentersOffices/CDRH/CDRHOffices/ucm115904.htm.

271 FDA: OIVD guidance. http://www.fda.gov/MedicalDevices/DeviceRegulationand Guidance/GuidanceDocuments/ucm070274.htm.

272 FDA: Guidance on pharmacogenetic tests and genetic tests for heritable markers. http://www.fda.gov/MedicalDevices/DeviceRegulationandGuidance/GuidanceDocuments/ ucm077862.htm.

273 HHS, FDA: FDA Draft guidance for industry, clinical laboratories, and FDA staff - In Vitro diagnostic multivariate index assays. http:/www.fda.gov/downloads/Medical Devices/DeviceRegulationandGuidance/GuidanceDocuments/ucm071455.pdfCDRH, 2006.

274 Secretary's Advisory Committee on Genetics, Health, and Society: U.S. System of Oversight of Genetic Testing: A Response to the Charge of the Secretary of Health and Human Services. Washington, DC: Department of Health and Human Services, 2008, http://oba.od.nih.gov/oba/SACGHS/reports/SACGHS_oversight_report.pdf.

275 OECD: OECD Guidelines for quality assurance in molecular genetic testing. http:// www.eurogentest.org/web/files/public/QAGuidelineseng.pdf.

276 Council of Europe: Additional Protocol to the Convention on Human Rights and Biomedicine, concerning Genetic Testing for Health Purposes. Strasbourg: Council of Europe, 2008. http://conventions.coe.int/treaty/EN/Treaties/Html/203.htm.

277 Jensen K, Murray F: Intellectual property landscape of the human genome. Science 2005; 310: 239-240.

278 Cho MK, Illangasekare S, Weaver MA, Leonard DGB, Merz JF: Effects of patents and licenses on the provision of clinical genetic testing services. JMD 2003; 5: 3-8.

279 Verbeure B, Matthijs G, van Overwalle G: Analysing DNA patents in relation with diagnostic genetic testing. Eur J Hum Genet 2006; 14: 26-33.

280 National Research Council: Reaping the Benefits of Genomic and Proteomic Research: Intellectual Property Rights, Innovation and Public Health. Washington: The National Academies Press, 2005

281 Barton JH: Emerging patent issues in genomic diagnostics. Nat Biotechnol 2006; 24 939-941.

282 Zika E, Gurwitz D, Ibarreta D: Pharmacogenetics and pharmacogenomics: state-ofthe-art and potential socio-economic impact in the EU. Luxembourg: The European Commission, 2006 (EUR 22214 EN).

283 Nuffield Council on Bioethics: Pharmacogenetics: Ethical Issues. London: Nuffield Council on Bioethics, 2003.

284 Balmaña J, Sanz J, Bonfill X et al: Genetic counseling program in familial breast cancer: analysis of its effectiveness, cost and cost-effectiveness ratio. Int J Cancer 2004; 112: 647-652.

285 Brain K, Gray J, Norman P et al: Randomized trial of a specialist genetic assessment service for familial breast cancer. I Natl Cancer Inst 2000; 92. 1345-1351.

286 Elkin EB, Weinstein MC, Winer EP, Kuntz KM, Schnitt SJ, Weeks JC: HER-2 testing and trastuzumab therapy for metastatic breast cancer: a cost-effectiveness analysis. $J$ Clin Oncol 2004; 22: 854-863.

287 Grann VR, Whang W, Jacobson JS, Heitjan DF, Antman KH, Neugut Al: Benefits and costs of screening Ashkenazi Jewish women for BRCA1 and BRCA2. J Clin Oncol 1999; 17: 494-500.

288 Heimdal K, Maehle L, Moller P: Costs and benefits of diagnostic familial breast cancer. Dis Markers 1999; 15: 167-173.

289 Morelle M, Haslé E, Treilleux I et al: Cost-effectiveness analysis of strategies for HER2 testing of breast cancer patients in France. Cost-effectiveness analysis of strategies for HER2 testing of breast cancer patients in France. Int J Technol Assess Health Care 2006; 22: 396-401.

290 Neyt MJ, Albrecht JA, Clarysse B, Cocquyt VF: Cost-effectiveness of Herceptin: a standard cost model for breast-cancer treatment in a Belgian university hospital. Int J Technol Assess Health Care 2005; 21: 132-137.
291 Norum J, Risberg T, Olsen JA: A monoclonal antibody against HER-2 (trastuzumab) for metastatic breast cancer: a model-based cost-effectiveness analysis. Ann Oncol 2005; 16: 909-914.

292 Sevilla C, Moatti JP, Julian-Reynier C et al: Testing for BRCA1 mutations: a costeffectiveness analysis. Eur J Hum Genet 2002; 10: 599-606

293 Tengs BO, Berry DA: The cost effectivenesss of testing for the BRCA1 and BRCA2 breast-ovarian cancer susceptibility genes. Dis Manag Clin Outcomes 2000; 1: 15-24.

294 Bapat B, Noorani H, Cohen Z et al: Cost comparison of predictive genetic testing versus conventional clinical screening for familial adenomatous polyposis. Gut 1999; 44: 698-703.

295 Breheny N, Geelhoed E, Goldblatt J, Ee H, O'Leary P: Economic evaluation of the familial cancer programme in Western Australia: predictive genetic testing for familial adenomatous polyposis and hereditary non-polyposis colorectal carcinoma. Community Genet 2006; 9: 98-106.

296 Chikhaoui Y, Gélinas H, Joseph L, Lance JM: Cost-minimization analysis of genetic testing versus clinical screening of at-risk relatives for familial adenomatous polyposis. Int J Technol Assess Health Care 2002; 18: 67-80.

297 Cromwell DM, Moore RD, Brensinger JD, Petersen GM, Bass EB, Giardiello FM: Cost analysis of alternative approaches to colorectal screening in familial adenomatous polyposis. Gastroenterology 1998; 114: 893-901.

298 Kievit W, de Bruin JH, Adang EM et al: Cost effectiveness of a new strategy to identify HNPCC patients. Gut 2005; 54: 97-102.

299 Ramsey SD, Clarke L, Etzioni R, Higashi M, Berry K, Urban N: Cost-effectiveness of microsatellite instability screening as a method for detecting hereditary nonpolyposis colorectal cancer. Ann Intern Med 2001; 135: 577-588.

300 Ramsey SD, Burke W, Clarke L: An economic viewpoint on alternative strategies for identifying persons with hereditary nonpolyposis colorectal cancer. Genet Med 2003 ; 5: 353-363.

301 Reyes CM, Allen BA, Terdiman JP, Wilson LS: Comparison of selection strategies for genetic testing of patients with hereditary nonpolyposis colorectal carcinoma: effectiveness and cost-effectiveness. Cancer 2002; 95: 1848-1856.

302 Vasen HF, van Ballegooijen M, Buskens E et al: A cost-effectiveness analysis of colorectal screening of hereditary nonpolyposis colorectal carcinoma gene carriers. Cancer 1998; 82: 1632-1637.

303 Marang-van de Mheen PJ, ten Asbroek AH, van Maarle MC, Stouthard MEA, Bonsel GJ, Klazinga NS: Screening op familiaire hypercholesterolemie in Nederland. Een evaluatie van kosten, effecten en maatschappelijke gevolgen. Project Zorgonderzoek Nederland-Praeventiefonds. Amsterdam: Academisch Medisch Centrum, 2000.

304 Marks D, Wonderling D, Thorogood M, Lambert H, Humphries SE, Neil HA: Screening for hypercholesterolaemia versus case finding for familial hypercholesterolaemia: a systematic review and cost-effectiveness analysis. Health Technol Assess 2000; 4: $1-123$.

305 Marks D, Wonderling D, Thorogood M, Lambert H, Humphries SE, Neil HAW: Cost effectiveness analysis of different approaches of screening for familial hypercholesterolaemia. BMJ 2002; 324: 1303-1308.

306 Wonderling D, Umans-Eckenhausen MAW, Marks D, Defesche JC, Kastelein JJP, Thorogood M: Cost-effectiveness analysis of the genetic screening program for familial hypercholesterolemia in the Netherlands. Semin Vasc Med 2004; 4: 97-104.

307 Adams PC, Valberg LS: Screening blood donors for hereditary hemochromatosis: decision analysis model comparing genotyping to phenotyping. Am J Gastroenterol 1999; 94: 1593-1600.

308 Bassett ML, Leggett BA, Halliday JW, Webb S, Powell LW: Analysis of the cost of population screening for haemochromatosis using biochemical and genetic markers. $J$ Hepatol 1997; 27: 517-524.

309 Gagné G, Reinharz D, Laflamme N, Adams PC, Rousseau F: Hereditary hemochromatosis screening: effect of mutation penetrance and prevalence on cost-effectiveness of testing algorithms. Clin Genet 2007; 71: 46-58.

310 Schöffski 0, Schmidtke J, Stuhrmann M: Cost-effectiveness of population-based genetic hemochromatosis screening. Community Genet 2000; 3: 2-11.

311 Auerbach AD, Sanders GD, Hambleton J: Cost-effectiveness of testing for hypercoagulability and effects on treatment strategies in patients with deep vein thrombosis. Am J Med 2004; 116: 816-828.

312 Creinin MD, Lisman R, Strickler RC: Screening for factor V Leiden mutation before prescribing combination oral contraceptives. Fertil Steril 1999; 72: 646-651.

313 Eckman MH, Singh SK, Erban JK, Kao G: Testing for factor V Leiden in patients with pulmonary or venous thromboembolism: a cost-effectiveness analysis. Med Decis Making 2002; 22: 108-124.

314 Marchetti M, Quaglini S, Barosi G: Cost-effectiveness of screening and extended anticoagulation for carriers of both factor $V$ Leiden and prothrombin G20210A. Q J Med 2001; 94: 365-372.

315 Marchetti M, Pistorio A, Barosi G: Extended anticoagulation for prevention of recurrent venous thromboembolism in carriers of factor $V$ Leiden-cost-effectiveness analysis. Thromb Haemost 2000; 84: 752-757.

316 Hahl J, Simell T, Ilonen J, Knip M, Simell O: Costs of predicting IDDM. Diabetologia 1998; 41: 79-85.

317 Ronteltap A, van Trijp JCM, Renes RJ, Frewer LJ: Consumer acceptance of technologybased food innovations: lessons for the future of nutrigenomics. Appetite 2007; 49: 1-17. 


\section{APPENDICES}

\section{A. ACCE - Criteria list for the evaluation of a genetic test}

\begin{tabular}{ll}
\hline Element & Sompecific question \\
\hline Disorder/setting & $1 . \quad$ What is the specific clinical disorder to be studied? \\
& $2 . \quad$ What are the clinical findings defining this disorder? \\
& $3 . \quad$ What is the clinical setting in which the test is to be performed? \\
& $4 . \quad$ What DNA test(s) are associated with this disorder? \\
& $5 . \quad$ Are preliminary screening questions employed? \\
& $6 . \quad$ Is it a stand-alone test or is it one of a series of tests? \\
$7 . \quad$ If it is part of a series of screening tests, are all tests performed in all instances (parallel) or are only
\end{tabular}

Analytic validity

Sensitivity Specificity

Clinical validity

Sensitivity

Specificity

Prevalence

8. Is the test qualitative or quantitative?

9. How often is the test positive when a mutation is present?

10. How often is the test negative when a mutation is not present?

11. Is an internal QC program defined and externally monitored?

12. Have repeated measurements been made on specimens?

13. What is the within- and between-laboratory precision?

14. If appropriate, how is confirmatory testing performed to resolve false-positive results in a timely manner?

15. What range of patient specimens have been tested?

16. How often does the test fail to give a useable result?

17. How similar are results obtained in multiple laboratories using the same or different technology?

Clinical utility

Intervention
Intervention
Intervention
Intervention
Intervention
Quality assurance
Pilot trials
Health risks
Economic
Facilities
Education
Monitoring

26. What is the natural history of the disorder?

27. What is the impact of a positive (or negative) test on patient care?

28. If applicable, are diagnostic tests available?

29. Is there an effective remedy, acceptable action, or other measurable benefit?

30. Is there general access to that remedy or action?

31 . Is the test being offered to a socially vulnerable population?

32. What quality assurance measures are in place?

33. What are the results of pilot trials?

34. What health risks can be identified for follow-up testing and/or intervention?

35. What are the financial costs associated with testing?

36. What are the economic benefits associated with actions resulting from testing?

37. What facilities/personnel are available or easily put in place?

38. What educational materials have been developed and validated and which of these are available?

39. Are there informed consent requirements?

40. What methods exist for long-term monitoring?

41. What guidelines have been developed for evaluating program performance?

$E L S I$

Impediments

42. What is known about stigmatization, discrimination, privacy/confidentiality, and personal/family social issues?

43. Are there legal issues regarding consent, ownership of data and/or samples, patents, licensing, proprietary testing, obligation to disclose, or reporting requirements? 
B. CHEC - Criteria list for systematic reviews on economic evaluation studies

1. Is the study population clearly described?

2. Are competing alternatives clearly described?

3. Is a well-defined research question posed in answerable form?

4. Is the economic study design appropriate to the stated objective?

5. Is the chosen time horizon appropriate to include relevant costs and consequences?

6. Is the actual perspective chosen appropriate?

7. Are all important and relevant costs for each alternative identified?

8. Are all costs measured appropriately in physical units?

9. Are costs valued appropriately?

10. Are all important and relevant outcomes for each alternative identified?

11. Are all outcomes measured appropriately?

12. Are outcomes valued appropriately?

13. Is an incremental analysis of costs and outcomes of alternatives performed?

14. Are all future costs and outcomes discounted appropriately?

15. Are all important variables, whose values are uncertain, appropriately subjected to sensitivity analysis?

16. Do the conclusions follow from the data reported?

17. Does the study discuss the generalizability of the results to other settings and patient/client groups?

18. Does the article indicate that there is no potential conflict of interest of study researcher(s) and funder(s)?

19. Are ethical and distributional issues discussed appropriately?

Source: Evers et al. ${ }^{165} 2005$ (C) Cambridge Journals, reproduced with permission. For a detailed description of the issues in the questions, see http://www.beoz.unimaas.nl/chec.

\section{Details on the systematic search}

C1: Search strategy

Database: PubMed

Date of query: 25 July 2007

\begin{tabular}{llc}
\hline Search no. & Query & Results \\
\hline 1 & Polymorphism, genetic[mesh] & 104421 \\
2 & Costs and cost analysis[mesh] OR economics[subheading] & 264416 \\
3 & Polymorphism, genetic[mesh] AND (costs and cost & 225 \\
& analysis[mesh] OR economics[subheading]) & \\
\hline
\end{tabular}

Database: Cochrane (including the databases of the Centre for Reviews and Dissemination (CRD): DARE, NHS EED, HTA)

Date of query: 2 August 2007

\begin{tabular}{llr}
\hline Search no. & Query & Results \\
\hline 1 & Polymorphism, genetic & 362 \\
2 & Economics & 183 \\
3 & Costs and cost analysis & 2457 \\
4 & Polymorphism, genetic AND economics & 12 \\
5 & Polymorphism, genetic AND costs and cost analysis & 203 \\
\hline
\end{tabular}

\section{C2: Studies identified}

It needs to be noted that this overview only presents the base case results calculated by the authors, without further assessment of quality. Many of these results are subject to high uncertainty and may underestimate the cost per health gain. For reviews discussing these studies, see, for example, Carlson et al, ${ }^{163}$ Jarrett and Mugford, ${ }^{170}$ and Rogowski. ${ }^{153}$

Breast and ovarian cancer

\begin{tabular}{|c|c|c|}
\hline Study & Result & $\begin{array}{l}\text { Type of mutation } \\
\text { or condition }\end{array}$ \\
\hline Balmaña et al ${ }^{284}$ & $€ 4,294 / \mathrm{LYG}$ & BRCA \\
\hline Brain et al 285 & $\begin{array}{l}\text { Additional } \$ 22.55 \text { for an initial } \\
\text { specialist genetic assessment } \\
\text { (vs standard care) }\end{array}$ & BRCA \\
\hline Elkin et al 286 & $\begin{array}{l}\text { \$145 000/QALY (FISH); } \$ 125000 / \\
\text { QALY (Hercep Test+FISH) }\end{array}$ & HER2 \\
\hline Grann et al ${ }^{287}$ & $\$ 20717 / L Y G$ & BRCA \\
\hline Heimdal et al 288 & $€ 832 / L Y G$ & BRCA \\
\hline Morelle et al 289 & Dominated $^{\mathrm{a}}$ & HER2 \\
\hline Neyt et al 290 & $\sim € 50000 / L Y G$ & HER2 \\
\hline Norum et al 291 & Not favorable & HER2 \\
\hline Sevilla et al292 & $\$ 971.30 /$ mutation detected & BRCA \\
\hline Tengs and Berry 293 & $<34000 / Q A L Y$ & BRCA \\
\hline
\end{tabular}

${ }^{a}$ An intervention is dominated by an alternative that can be implemented in a more effective way and at lower costs at the same time.

Colorectal cancer

\begin{tabular}{|c|c|c|}
\hline Study & Result & $\begin{array}{l}\text { Type of mutation } \\
\text { or condition }\end{array}$ \\
\hline Bapat et al 294 & \$2568/family saved (CAN\$3056) & FAP \\
\hline Breheny et al 295 & AU\$12 141-14 783/person saved & HNPCC \& FAP \\
\hline Chikhaoui et al 296 & $\begin{array}{l}\text { Cost saving (vs clinical screening } \\
\text { without prior genetic testing) }\end{array}$ & FAP \\
\hline Cromwell et al 297 & $\$ 583 /$ screened person saved & FAP \\
\hline Kievit et al 298 & $€ 2184 / L Y G$ & HNPCC \\
\hline Ramsey et al299 & $\begin{array}{l}\$ 42210 / \text { YYG (probands tested); } \$ 7556 / \text { YYG } \\
\text { (probands and siblings and children tested) }\end{array}$ & HNPCC \\
\hline Ramsey et al ${ }^{300}$ & $\begin{array}{l}\$ 11865 / \text { LYG }(\$ 8005-\$ 80226 / L Y G) \\
\text { (Bethesda guidelines treatment algorithm); } \\
\$ 35617 / \text { LYG ( } \$ 15091-\$ 180056 / \mathrm{LYG} \text { ) (MSI } \\
\text { testing to detect HNPCC carriers); } \$ 49702 / \mathrm{LYG} \\
\text { (\$19 100-\$252 151/LYG) (DNA analysis for } \\
\text { Bethesda clinical positive points); } \$ 267 \\
\text { 548/LYG ( } \$ 68328-\$ 637007 / \mathrm{LYG} \text { ) (DNA } \\
\text { analysis for all) }\end{array}$ & HNPCC \\
\hline Reyes et $a l^{301}$ & $\$ 6441 /$ mutation detected & HNPCC \\
\hline Vasen et $a l^{302}$ & $\$ 12577 / L Y$ & HNPCC \\
\hline
\end{tabular}

Familial hypercholesterolemia

\begin{tabular}{|c|c|c|}
\hline Study & Result & $\begin{array}{l}\text { Type of mutation or } \\
\text { condition }\end{array}$ \\
\hline $\begin{array}{l}\text { Marang-van de } \\
\text { Mheen et al }{ }^{303}\end{array}$ & $€ 25000-32$ 000/LYG & LDL receptor \\
\hline Marks et al ${ }^{304}$ & $\begin{array}{l}\text { Dominated (by cholesterol testing and } \\
\text { phenotype diagnosis) }\end{array}$ & LDL receptor \\
\hline Marks et $a l^{305}$ & $\begin{array}{l}\text { Dominated (by cholesterol testing and } \\
\text { phenotype diagnosis) }\end{array}$ & LDL receptor \\
\hline Wonderling et al ${ }^{306}$ & $\begin{array}{l}\text { \$87 000/LYG (worst-case scenario: } \\
\$ 38300 / \text { LYG) }\end{array}$ & LDL receptor \\
\hline
\end{tabular}


Hemochromatosis

\begin{tabular}{|c|c|c|}
\hline Study & Result & $\begin{array}{c}\text { Type of mutation } \\
\text { or condition }\end{array}$ \\
\hline Adams and Valberg 307 & Dominated (by phenotypic screening) & HFE \\
\hline Bassett et $a l^{308 *}$ & $>\$ 669$ savings per case detected & HLA \\
\hline El-Serag et al ${ }^{133 *}$ & $\$ 508-3665 / L Y G$ & HFE \\
\hline Gagné et al 309 & Potentially cost saving & HFE \\
\hline Schöffski et a/ ${ }^{310}$ & $€ 4441 / L Y G$ & HFE \\
\hline
\end{tabular}

A recent study published after this review concluded that the cost effectiveness of population screening for hereditary hemochromatosis in Germany is about 124000 and 161000 EUR/LYG for phenotype and genotype screening, respectively. ${ }^{145}$

Thrombotic disease

\begin{tabular}{|c|c|c|}
\hline Study & Result & $\begin{array}{l}\text { Type of mutation or condi- } \\
\text { tion }\end{array}$ \\
\hline Auerbach et $\left.a\right|^{311}$ & $\begin{array}{l}\text { \$11 100/QALY (positive } \\
\text { tested individuals treated } \\
\text { for } 24 \text { months) }\end{array}$ & $\begin{array}{l}\text { FVL, prothrombin } \\
\text { (G20210A)+antithrombin } \\
\text { III+protein C+protein S, } \\
\text { homocysteinemia+lupus } \\
\text { anticoagulant }\end{array}$ \\
\hline Creinin et $a l^{312}$ & $\begin{array}{l}\$ 4800000 / \text { life year } \\
\text { (for a } 20 \text {-year-old woman); } \\
\$ 7100000 / \text { life year } \\
\text { (for a } 40 \text {-year-old woman) }\end{array}$ & $\begin{array}{l}\text { FVL+activated protein C, } \\
\text { protein S+antithrombin III } \\
\text { deficiencies }\end{array}$ \\
\hline Eckman et al ${ }^{313}$ & $\$ 16823 /$ QALY & FVL+activated protein C \\
\hline Marchetti et al ${ }^{314}$ & $\$ 13624 /$ QALY & $\begin{array}{l}\text { FVL+prothrombin } \\
\text { (G20210A) }\end{array}$ \\
\hline Marchetti et al ${ }^{315}$ & $\$ 12833 /$ QALY & $\begin{array}{l}\text { FVL+prothrombin } \\
\text { (G20210A) }\end{array}$ \\
\hline
\end{tabular}

Diabetes mellitus

\begin{tabular}{lll}
\hline Study & Result & $\begin{array}{l}\text { Type of mutation or } \\
\text { condition }\end{array}$ \\
\hline Hahl et al ${ }^{316}$ & $\begin{array}{l}\text { Cost saving (vs repeated analy- } \\
\text { sis of markers of autoimmunity } \\
\text { in the entire population) }\end{array}$ & $\begin{array}{l}\text { HLA-DQB1 } \\
\text { (type } 1 \text { diabetes) }\end{array}$ \\
\hline
\end{tabular}

\section{Nutrigenomics}

D1: Search strategy

Database: PubMed

Date of query: 28 August 2007

\begin{tabular}{llc}
\hline Search no. & Query & Results \\
\hline 1 & Nutrigenomics & 134 \\
2 & Costs and cost analysis[mesh] OR economics[subheading] & 264416 \\
3 & Nutrigenomics AND (costs and cost analysis[mesh] OR & 1 \\
& economics[subheading]) & \\
\hline
\end{tabular}

Database: Cochrane (including the databases of the Centre for Reviews and Dissemination (CRD): DARE, NHS EED, HTA)

Date of query: 28 August 2007

\begin{tabular}{llc}
\hline Search no. & Query & Results \\
\hline 1 & Nutrigenomics & 1 \\
2 & Economics & 183 \\
3 & Costs and cost analysis & 2457 \\
4 & Nutrigenomics AND economics & 0 \\
5 & Nutrigenomics AND costs and cost analysis & 0 \\
\hline
\end{tabular}

D2: Studies identified

\begin{tabular}{lll}
\hline Study & Details & Source \\
\hline $\begin{array}{lll}\text { Ronteltap } \\
\text { et } \text { l }^{317}\end{array}$ & $\begin{array}{l}\text { Consumer acceptance of technology-based food } \\
\text { interventions }\end{array}$ & $\begin{array}{l}\text { Supplementary } \\
\text { search }\end{array}$ \\
\hline
\end{tabular}




\section{E. Genetic tests available online}

Table 5 Direct-to-consumer (DTC) testing companies

Direct-to-consumer (DTC) testing companies

\begin{tabular}{|c|c|c|}
\hline Company & Tests offered & Delivery model \\
\hline 23andMe & $\begin{array}{l}\text { Susceptibility testing for common diseases as well as } \\
\text { ancestry testing }\end{array}$ & DTC via internet \\
\hline Acu-Gen Biolab, Inc. & Fetal DNA gender test & DTC via internet \\
\hline Consumer genetics & $\begin{array}{l}\text { Fetal gender; caffeine metabolism; alcohol metabolism; } \\
\text { asthma drug response }\end{array}$ & DTC via internet \\
\hline Cygene Direct & $\begin{array}{l}\text { Osteoporosis; athletic performance; glaucoma and } \\
\text { macular degeneration; thrombosis }\end{array}$ & DTC via internet \\
\hline deCODE (Iceland) & $\begin{array}{l}\text { Susceptibility testing for cancers, diabetes, heart dis- } \\
\text { ease, osteoporosis, and Parkinson's disease and others; } \\
\text { also ancestry testing }\end{array}$ & DTC via internet \\
\hline Dermagenetics & Skin DNA profile; custom skin cream & DTC through spas and similar retailers \\
\hline DNADirect & $\alpha-1$ antitrypsin deficiency; Ashkenazi Jewish carrier & DTC via internet; genetic counsellors available by phone \\
\hline
\end{tabular}

G-Nostics (UK)

Genelex

Genetic Health (UK) (tests are performed by an Austrian test developer and laboratory Genosense)

Geneticom (Netherlands)

Genosense (Austria)

Graceful Earth

Health Tests Direct

Health Check USA

Holistic Health

Kimball screening; blood clotting disorders; breast and ovarian cancer; colon cancer screening; cystic fibrosis; diabetes risk; drug response panel; hemochromatosis; infertility; recurrent pregnancy loss; tamoxifen

Predisposition to nicotine addiction and response to nicotine replacement products

Pharmacogenetics testing; celiac disease; hemochromatosis; gum disease; nutritional genetic testing; DNA Diet $^{\mathrm{TM}}$ consultation; weight loss system

For males: genetic predisposition to prostate cancer, thrombosis, osteoporosis, metabolic imbalances of detoxification, and chronic inflammation

For females: genetic predisposition to breast cancer, bone metabolism (osteoporosis), thrombosis, cancer, and long-term exposure to estrogens

Nutrigenetic test: test for a range of genes that influence nutritional processes such as lipid and glucose metabolism Pharmacogenetic test: test for CYP450 genes, which influence how the liver metabolizes a large number of commonly prescribed drugs

Premium male gene/premium female gene: combine all the other tests except the nutrigenetic one

Common disease risk

Susceptibility tests

Alzheimer (ApoE)

More than 400 blood tests, including a few genetic tests (cystic fibrosis carrier screen, Factor V Leiden); others may also be available by calling

A wide range of laboratory tests, including the following genetic tests: celiac disease; Factor V R2; Factor V Leiden; hereditary hemochromatosis

Nutrigenomic test: comprehensive methylation panel with methylation pathway analysis; company also sells a variety of nutritional supplements

Wide range of well-established genetic tests
DTC via internet; genetic counsellors available by phone

DTC via internet and through pharmacies

DTC via internet

DTC via internet; most services include a medical consultation

Not clear

Do not offer DTC tests themselves, but some of the institutions they partner with to order tests for consumers offer DTC testing (eg, Genetic Health in the United Kingdom)

DTC via internet

DTC via internet

DTC via internet; as additional service, the patient can request interpretation by a board-certified physician; free genetic counselling offered by Kimball Genetics for physicians, patients, and families Not described

DTC via internet, but detailed telephone consultation with certified genetic counsellor is mandatory; report is sent to the physician and the customer 
Table 5 Continued

Direct-to-consumer (DTC) testing companies

\begin{tabular}{|c|c|c|}
\hline Company & Tests offered & Delivery model \\
\hline MediChecks (UK) & $\begin{array}{l}\text { Wide range of well-established genetic tests, from Factor } \\
\mathrm{V} \text { thrombosis risk to BRCA testing for breast cancer risk } \\
\text { (most tests are performed by the private pathology } \\
\text { laboratory TDL) }\end{array}$ & $\begin{array}{l}\text { DTC via internet, but company recommends physician } \\
\text { referral for high-impact tests such as BRCA }\end{array}$ \\
\hline Medigenomix (Germany) & Thrombophilia and osteoporosis risk tests & DTC via internet \\
\hline Mygenome.com & $\begin{array}{l}\text { Alzheimer's disease (genetic testing for common risk } \\
\text { factors); drug sensitivities (genetic tests for genes that } \\
\text { affect the safety and activity of many common pre- } \\
\text { scription and over-the-counter drugs); cardiovascular } \\
\text { disease (genetic tests differentiate treatable risk factors } \\
\text { for heart disease and stroke); thrombosis (genetic tests } \\
\text { identify risk factors for blood clots); pregnancy risk } \\
\text { (genetic tests identify risk factors for complications of } \\
\text { pregnancy); osteoporosis (genetic tests identify risk fac- } \\
\text { tors for osteoporosis and fractures) }\end{array}$ & Not clear \\
\hline Navigenics & $\begin{array}{l}\text { Risk analysis for more than } 20 \text { common diseases, such } \\
\text { as prostate cancer and diabetes }\end{array}$ & DTC via internet \\
\hline Quixtar & $\begin{array}{l}\text { Heart health; nutrigenic tests and supplements; also } \\
\text { sells dietary supplement }\end{array}$ & DTC via internet \\
\hline Salugen & Nutrigenic tests and supplements & DTC sold through spas \\
\hline Sciona & $\begin{array}{l}\text { Heart health; bone health; insulin resistance; antioxi- } \\
\text { dant/detoxification; inflammation }\end{array}$ & DTC via Internet \\
\hline Smart Genetics & Prediction of HIV progression to AIDS & DTC via internet; free counselling available \\
\hline Suracell & $\begin{array}{l}\text { DNA profile test that identifies inherited genetic aging } \\
\text { profile, and a biomarker assessment test that measures } \\
\text { DNA damage, oxidative stress, and free radical levels; } \\
\text { personal genetic supplements for DNA repair and } \\
\text { nutrition }\end{array}$ & DTC via internet \\
\hline
\end{tabular}

Source: Reproduced with permission of Annual Reviews, Inc. from Hogarth et al. ${ }^{192}$ Copyright $(C) 2008$ by Annual Reviews, Inc.; permission conveyed through Copyright Clearance Center, Inc. 